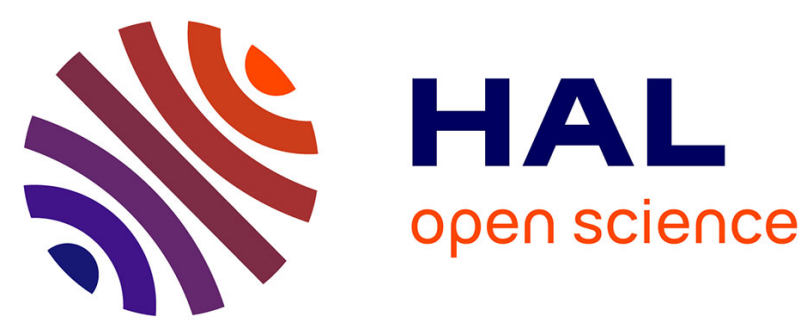

\title{
Tripodal molecules with triphenylamine core, diazine peripheral groups and extended $\pi$-conjugated linkers
}

Daniel Cvejn, Sylvain Achelle, Oldřich Pytela, Jean-Pierre Malval, Arnaud Spangenberg, Nolwenn Cabon, Filip Bureš, Françoise Robin-Le Guen

\section{- To cite this version:}

Daniel Cvejn, Sylvain Achelle, Oldřich Pytela, Jean-Pierre Malval, Arnaud Spangenberg, et al.. Tripodal molecules with triphenylamine core, diazine peripheral groups and extended $\pi$-conjugated linkers. Dyes and Pigments, 2016, 124, pp.101-109. 10.1016/j.dyepig.2015.09.012 hal-01205442

HAL Id: hal-01205442

https://hal-univ-rennes1.archives-ouvertes.fr/hal-01205442

Submitted on 21 Oct 2015

HAL is a multi-disciplinary open access archive for the deposit and dissemination of scientific research documents, whether they are published or not. The documents may come from teaching and research institutions in France or abroad, or from public or private research centers.
L'archive ouverte pluridisciplinaire HAL, est destinée au dépôt et à la diffusion de documents scientifiques de niveau recherche, publiés ou non, émanant des établissements d'enseignement et de recherche français ou étrangers, des laboratoires publics ou privés. 


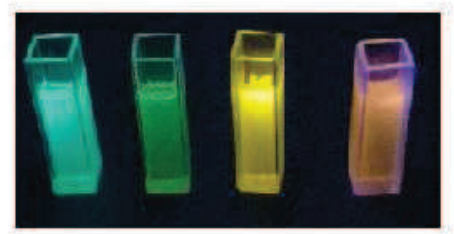

Intense fluorescence High emission solvatochromism

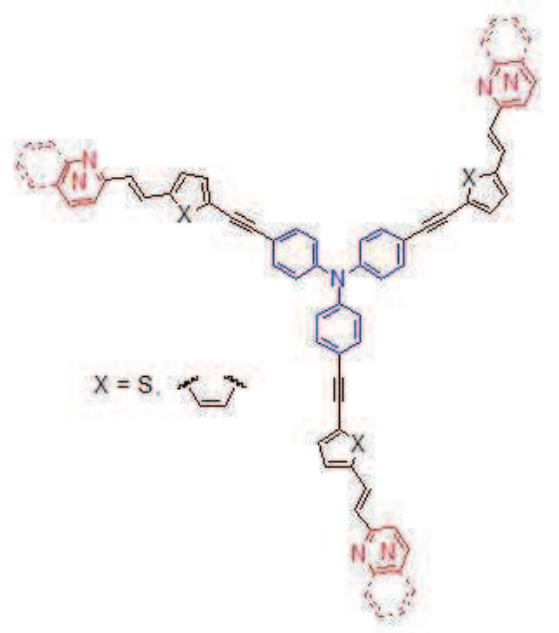

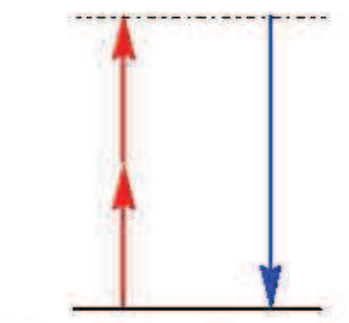

Two-photon absorption $\delta \max =158-427 \mathrm{GM}$ 


\title{
Tripodal molecules with triphenylamine core, diazine peripheral groups and extended $\pi$-conjugated linkers
}

Daniel Cvejn,${ }^{[\mathrm{a}, \mathrm{b}]}$ Sylvain Achelle, ${ }^{*[\mathrm{a}]}$ Oldřich Pytela, ${ }^{[\mathrm{b}]}$ Jean-Pierre Malval, ${ }^{[\mathrm{c}]}$ Arnaud Spangenberg, ${ }^{[\mathrm{c}]}$ Nolwenn Cabon, ${ }^{[\mathrm{a}]}$ Filip Bureš, ${ }^{* \mathrm{~b}]}$ and Françoise Robin-le Guen ${ }^{[\mathrm{a}]}$

${ }^{[a]}$ Institut des Sciences Chimiques de Rennes UMR CNRS 6226, IUT de Lannion, rue Edouard Branly, BP 30219, F22302 Lannion Cedex, France. ${ }^{[\mathrm{b}]}$ Institute of Organic Chemistry and Technology, Faculty of Chemical Technology, University of Pardubice, Studentská 573, Pardubice, 53210, Czech Republic. ${ }^{[c]}$ Institut de Science des Matériaux de Mulhouse UMR CNRS 7361, Université de Haute-Alsace 15 rue Jean Starcky, 68057 Mulhouse, France

Corresponding authors: *E-mails: sylvain.achelle@univ-rennes1.fr; filip.bures@upce.cz

\begin{abstract}
A series of five octupolar molecules with central triphenylamine donor and peripheral diazine electron-withdrawing groups has been prepared. These structures possess extended $\pi$-spacer comprising multiple bonds in a combination with 1,4-phenylene and 2,5-thienylene subunits. Starting from tris(4-ethynylphenyl)amine, the key step in the preparation of the chromophores involves triple Sonogashira cross-coupling reaction. The photophysical properties of the compounds are described. A strong positive emission solvatochromism, typical for dyes featuring Intramolecular Charge Transfer (ICT), is observed, two-photon absorption (2PA) properties were also measured. Linear and nonlinear optical properties as well as electronic
\end{abstract}


properties measured by cyclic voltametry and supported by DFT calculation were used to elucidate structure-property relationships.

Keywords: Diazines; Triphenylamines; Nonlinear Optics; Fluorescence; Intramolecular charge transfer

\section{Introduction}

During the past decade, organic molecules with a delocalized $\pi$-system of electron have been subject to numerous studies due to their prospective applications in field-effect transistor (OFETs), ${ }^{1}$ organic light-emitting diodes (OLEDs $)^{2}$ and photovoltaic devices. ${ }^{3}$ Among these systems, donor acceptor $\pi$-conjugated (D- $\pi$-A) materials, also called push-pull, represent a subclass with particular applications such as quadratic and cubic nonlinear optical (NLO) properties. ${ }^{4}$ Intrinsic NLO properties of organic push-pull molecules are mainly dictated by the extent of ICT, which is originated by the D-A interaction and can easily be illustrated by two limiting resonance forms (aromatic and quinoid/zwitterionic arrangements). ${ }^{5}$ In contrast to ordinary linear D- $\pi$-A molecules, star-shaped molecules with $\mathrm{D}(-\pi-\mathrm{A})_{3}$ or $\mathrm{A}(-\pi-\mathrm{D})_{3}$ arrangements exhibit enhanced ICT and further peculiar properties due to their unique structures and manifold transitions. ${ }^{6}$ Branching of a push-pull molecule helps to reduce aggregation and to maintain luminescence in the solid state. Star-shaped structures are also known to bring cooperative enhancement in terms of conjugation expansion and twophoton absorption (2PA) properties. ${ }^{7}$ Among others, triphenylamine represent undoubtedly the most frequent precursor used for the construction of $\mathrm{D}(-\pi-\mathrm{A})_{3}$ molecules and its derivatives have been widely applied as 2PA-active substances. Triphenylamine core is considered as a moderate electron donor with propeller-shaped arrangement and $C_{3}$ symmetry and, therefore, displays an octupolar feature. Acceptor terminated triphenylamine derivatives were described as excellent 2PA chromophores. ${ }^{8}$ Such chromophores have found applications 
for 2PA fluorescent microscopy, ${ }^{9}$ cellular bioimaging, ${ }^{10}$ 2PA-photodynamic therapy, ${ }^{11} 3 \mathrm{D}$ optical data storage, ${ }^{12}$ microfabrication, ${ }^{13}$ three-photon absorbers, ${ }^{14}$ organic solar cells, ${ }^{15}$ molecular glasses, ${ }^{16}$ guest molecules for intercalation and metal-organic frameworks (MOF) $)^{17}$ as well as various detectors and sensors. ${ }^{18}$

Pyrimidine, pyrazine and quinoxaline (benzopyrazine) rings belong to the diazine heterocycles, which have been widely used as electron-withdrawing moieties in chargetransfer chromophores. ${ }^{19}$ In particular, the 4,6-di(arylvinyl)pyrimidine motif has been synthetically well-established and push-pull molecules based on this scaffold delivered large 2PA cross-section. $^{20}$ Linear charge-transfer chromophores bearing diazine ring were investigated for their fluorescence, ${ }^{21} 2^{\text {nd }}$ order $\mathrm{NLO}^{22}$ and $2 \mathrm{PA}$ properties. ${ }^{23}$ Recently, Martin and coworkers have reported some star-shaped molecules with triphenylamine core and pyrimidine and pyridazine rings as peripheral groups and some of these tripodal molecules exhibited promising 2PA cross-section. ${ }^{24}$ In general, the electron donor and acceptor as well as the $\pi$-conjugated bridge are integral parts of the D- $\pi$-A system and their optimization represents a key parameter to enhance the ICT and maximize the fluorescence intensity and 2PA cross-section. ${ }^{5}$ Hence, the main purpose of the present article is to design and synthesize new star-shaped molecules 1-5 based on triphenylamine equipped with diazine peripheral acceptors and extended $\pi$-conjugated linkers (Chart 1). Influence of the length, planarity and composition of the $\pi$-linker as well as the nature of the diazine rings (nitrogen atom topology and further extension) on the photophysical and electronic properties will be thoroughly studied.

\section{$<$ Insert Chart 1.>}

\section{Results and discussion}


Preparation of star-shaped molecules. Arylvinyldiazines can easily be obtained by Knoevenagel condensation of corresponding aldehyde with commercially available methyldiazines. Vanden Eynde and co-workers described the synthesis of arylvinyldiazines using boiling aqueous $5 \mathrm{M} \mathrm{NaOH}$ in the presence of Aliquat 336 as a phase-transfer catalyst. ${ }^{25}$ These conditions proved to be very efficient in the case of 4-methylpyrimidine and 2-methylquinoxaline and smoothly afforded bromo-substituted intermediates 6-9 in the yields of 54-79\% (Scheme 1). On the contrary, using 2-methylpyrazine and similar reaction condition provided only traces of the desired arylvinyldiazine 10. This is most likely due to a lower acidity of the hydrogens appended at the methyl group. However, potassium hydroxide in DMSO (dimsyl potassium) at room temperature proved to be a sufficient base to abstract 2-methylpyrazine hydrogens ${ }^{26}$ and subsequent reaction with 5-bromothiophene-2carbaldehyde afforded compound $\mathbf{1 0}$ (Scheme 2). In this way, compound $\mathbf{1 0}$ was obtained in good yield and its purification was facile. Stereochemical outcome of the aforementioned Knoevenagel reactions was verified by ${ }^{1} \mathrm{H}$ NMR spectroscopy where all intermediates 6-10 showed pair of doublets with the coupling constants ${ }^{3} J(\mathrm{H}, \mathrm{H})$ about $16 \mathrm{~Hz}$, which clearly evidences selective formation of $(E)$-configured compounds $\mathbf{6 - 1 0} .^{27}$

\section{$<$ Insert Scheme 1> \\ $<$ Insert Scheme 2>}

Arylvinyldiazines 6-10 were further used as coupling partners for the construction of target molecules 1-5 (Scheme 3). Threefold Sonogashira cross-coupling reaction with tris(4ethynylphenyl)amine (11) $)^{8 \mathrm{a}}$ carried out with $\mathrm{Pd}(0)$-precatalysts, diisopropylamine, 1,4-dioxane and the temperature elevated up to $92{ }^{\circ} \mathrm{C}$ afforded star-shaped chromophores 1-5. Besides tripodal molecules 1-5, a formation of twofold and single cross-coupled products was 
also observed and could partially be suppressed by an excess of bromo derivatives 6-10. However, the relatively low isolated yields of $23-60 \%$ are mainly caused by difficult separation of 1-5 from the side products by column chromatography (repeated purifications were required). All of the new materials exhibited good solubility in a variety of solvents, especially in THF and chlorinated solvents. The ${ }^{1} \mathrm{H},{ }^{13} \mathrm{C}$ NMR and HRMS data were consistent with the expected structures.

\section{$<$ Insert Scheme 3>}

UV-vis and Fluorescence Spectroscopy. The UV-Vis and photoluminescence (PL) spectroscopic data of star-shaped molecules 1-5, measured in dichloromethane and in toluene at $25{ }^{\circ} \mathrm{C}$, are presented in Tables 1 and S8. The analyses were carried out using low concentrated solutions (1.0 to $2.0 \times 10^{-5} \mathrm{M}$ for UV/Vis spectra and 1.0 to $3.0 \times 10^{-6} \mathrm{M}$ for PL spectra). As an example, the spectra for derivatives $\mathbf{1}$ and $\mathbf{4}$ in dichloromethane are shown in Figure 1. Under these conditions, self-absorption effects were not observed. All compounds proved to be photostable and none $E$ to $Z$ isomerization was observed under the given conditions.

$<$ Insert Table 1 $>$

$<$ Insert Figure 1 >

In dichloromethane, all target compounds exhibited absorption in visible region with the position of the longest-wavelength absorption maxima $\left(\lambda_{\max }\right)$ appearing at 401 to $436 \mathrm{~nm}$. In all cases a second particularly developed band of higher energy was also observed. Molecular extinction coefficients of $\mathbf{1 - 5}\left(\varepsilon=74.5\right.$ to $\left.108.3 \mathrm{mM}^{-1} \cdot \mathrm{cm}^{-1}\right)$ are approximately two to three times higher than that of corresponding linear molecules. ${ }^{22 a}$ Depending on the compound used, the emission maxima were observed in the green to orange region $\left(\lambda_{\max }=555-630 \mathrm{~nm}\right)$. Interestingly, fluorescence 
quantum yields are relatively high $\left(\Phi_{\mathrm{F}}=0.37-0.67\right)$ even though the emission is red-shifted. Nevertheless, except for compound 2, the fluorescence quantum yields are higher in toluene than in dichloromethane: a quantum yield up to 1.00 was observed for compound $\mathbf{1}$ in toluene. As expected for donor-acceptor functionalized $\pi$-conjugated molecules featuring the ICT, large Stokes shifts (up to $8340 \mathrm{~cm}^{-1}$ ) were observed, which indicates large differences (vibrational, electronic, geometric) between the Franck-Condon state and the emissive excited state.

When comparing the nature of the peripheral diazine ring, the most red-shifted emissions were observed for quinoxaline compounds $\mathbf{3}$ and $\mathbf{4}$, whereas the most hypsochromically shifted emission was observed for pyrazine substituted compound 5. A similar trend was observed previously. ${ }^{22 e}$ The highest fluorescence quantum yield in dichloromethane is observed for pyrazine derivative $\mathbf{5}$.

In general, replacement of 1,4-phenylene moieties in compounds $\mathbf{1}$ and $\mathbf{3}$ by 2,5-thienylene rings (compounds 2 and 4) led to a red shift of both absorption and emission spectra. This is in accordance with the previous observation on linear push-pull chromophores. ${ }^{28,5}$ However, in case of quinoxaline derivatives $\mathbf{3}$ and $\mathbf{4}$, in dichloromethane, a blue-shifted emission is observed for thienylene derivative 4 .

In an effort to gain further insights into the photophysical process within these push-pull molecules, the absorption and emission behavior of the compounds $\mathbf{1 - 5}$ was studied in a variety of different aprotic solvents. While the positions of the longest-wavelength absorption maxima were not significantly affected, an increase in the solvent polarity led to bathochromic shifts of the emission maxima (Table 2). This behavior is generally observed for chromophores with ICT. ${ }^{7 \mathrm{~d}, 9 \mathrm{~b}, 9 \mathrm{~d}, 21,22 \mathrm{a}}$ As an example, the spectra recorded for compound 2 are shown in Figure 2. When going from the least polar hexane $\left(\lambda_{\mathrm{em}}=468 \mathrm{~nm}\right)$ to toluene, dioxane, THF, $\mathrm{CH}_{2} \mathrm{Cl}_{2}$ and acetone, the emission maxima was considerably red-shifted up to $618 \mathrm{~nm}\left(\Delta \lambda_{\mathrm{em}}=150 \mathrm{~nm}\right.$ resp. $\left.\Delta v_{\mathrm{em}} 5186 \mathrm{~cm}^{-1}\right)$. In more polar solvent such as MeCN or 
DMSO, the emission intensity was low and less detectable. When increasing the polarity, the emission spectra are less structured and emission bands are larger. To further illustrate strong emission solvatochromism of target star-shaped chromophores, Figure 3 shows the emission color of $\mathbf{4}$ in different solvents. Such behavior is consistent with a stabilization of the highly polar emitting excited states by polar solvents and has also been observed either for triphenylamine derivatives bearing electron-withdrawing groups ${ }^{8 b, 9,29}$ or for diazines substituted with electron-donating substituents. ${ }^{21,22 a, 22 e, 30}$ The emission solvatochromism represents one of the possible ways to evaluate ICT upon excitation in fluorescent molecules. Hence, the emission maxima were plotted versus the $\mathrm{E}_{\mathrm{T}}(30)$ Dimroth-Reichardt polarity parameters $^{31}$ (see the Supporting Information). For compounds 1-3 are the slope of the regression line slightly higher than for their linear analogues indicating a branching effect that enhances the $\mathrm{ICT}^{22 \mathrm{a}}$ When comparing the different diazine rings, quinoxaline derivatives $\mathbf{3}$ and 4 exhibited the highest regression line slopes. A replacement of 1,4-phenylene by 2,5thienylene units in compounds $\mathbf{2}$ and $\mathbf{4}$ versus $\mathbf{1}$ and $\mathbf{3}$ tends to reduce the regression line slope and, therefore, chromophore $\mathbf{3}$ showed the highest regression line slope. When compared with star-shaped triphenylamine derivatives equipped with trifluoromethanesulfonate acceptors and similar $\pi$-conjugated linkers, the quinoxaline derivatives $\mathbf{3}$ and $\mathbf{4}$ showed very similar solvatochromic behavior. ${ }^{32}$

\section{$<$ Insert Table $2>$ \\ $<$ Insert Figure $2>$ \\ $<$ Insert Figure $3>$}

Dichloromethane solutions of compounds 1-5 underwent an important color change upon addition of trifluoroacetic acid (TFA) with an important bathochromic shift of the absorption spectra (Figures 4-5). We had already observed this phenomenon, which is due to the protonation of the diazine ring that enhances its electron-withdrawing character and 
reinforce the ICT into the molecule. ${ }^{21 \mathrm{a}, \mathrm{c}, \mathrm{d}, 22 \mathrm{c}, \mathrm{e}, 33}$ These molecules appear therefore as potential colorimetric $\mathrm{pH}$ sensors. However, it should be noted that these color changes appear at higher concentration of TFA $\left(10^{-2}-10^{-1} \mathrm{M}\right)$ than for other diazine derivatives with shorter $\pi$-conjugated linker $\left(10^{-5}-10^{-3} \mathrm{M}\right){ }^{34}$ On the other hand, the emission was totally quenched after treatment with acid, as is generally observed for amino electron donor substituted diazines. $^{21 \mathrm{a}, \mathrm{d}, 34}$

$<$ Insert Figure $4>$

$<$ Insert Figure $5>$

Two photon absorption measurements. 2PA data of chromophores 1-5, measured by relative two-photon excited fluorescence (2PEF) method, are presented in Table 3, and the 2PA spectra are shown in Figure 6. According to our spectral resolution, all octupoles exhibit a broad 2PA band whose maximum absorption wavelength is slightly lower than twice $\lambda_{\max }$ of the one photon absorption (1PA) band (see supporting information). The 2PA cross section $\left(\delta_{\max }\right)$ are comprised between 158 and 427 GM. It is noteworthy that the thiophene-based derivatives 2 and 4 present another distinctive 2PA band around $940 \mathrm{~nm}$. According to the Frenkel exciton model, ${ }^{34}$ all those spectral effects should be ascribed to a significant interbranch coupling within these multipolar chromophores which leads to a splitting of the initially degenerate single excited levels associated to each molecular synthon. ${ }^{7 \mathrm{~d}, 13 \mathrm{a}, 35}$ Such a coupling is consequently responsible for the presence of multiple electronic $\pi-\pi^{*}$ transitions within the longest wavelength of the 1PA band as previously observed for similar trigonal branched structures. $^{7 \mathrm{~d}, 13 \mathrm{a}, 35 \mathrm{a}, 36,36}$ It should be noted that the $2 \mathrm{PA}$ cross sections are clearly enhanced on going from 1,4-phenylene group used as 'electron relay' to a 1,2-thienylene one (compounds 1 and $\mathbf{3}$ versus $\mathbf{2}$ and $\mathbf{4}$ ). As expected, changing the pyrimidine end groups by pyrazine ones induces a noticeable decrease of $\delta_{\max }$ (chromophores $\mathbf{1}$ versus $\mathbf{5}$ ). However, 
interestingly, quinoxaline (benzopyrazine) derivatives 3 and $\mathbf{4}$ gave similar $\delta_{\max }$ values in comparison with their pyrimidine analogues $\mathbf{1}$ and $\mathbf{2}$. In term of action cross section, defined by the product of 2PA cross section and fluorescence quantum yield, compound $\mathbf{2}$ showed the highest figure of merit with $\Phi \times \delta_{\max }=248 \mathrm{GM}$.

\section{$<$ Insert Figure 6 > \\ $<$ Insert Table $3>$}

\section{Electrochemical properties}

Electrochemical behavior of chromophores $\mathbf{1 - 5}$ was investigated by cyclic voltammetry in $\mathrm{DMF}-\left[\mathrm{Bu}_{4} \mathrm{~N}\right]\left[\mathrm{PF}_{6}\right]$ at a scan rate of $0.1 \mathrm{~V} / \mathrm{s}$. The working electrode was a glassy carbon disk, tungsten wire was used as the counter electrode and a non aqueous $\mathrm{Ag} / \mathrm{Ag}^{+}$electrode was used as reference. A representative $\mathrm{CV}$ diagram of chromophore $\mathbf{5}$ is shown in the SI, and the voltammogram shows two oxidation and two reduction waves. The second oxidation and the second reduction are irreversible processes. The first oxidation/reduction potentials as well as the electrochemical gap $E \mathrm{~g}^{\text {elchem }}$ and recalculated energies of the HOMO and the LUMO are presented in Table 4.

The first oxidation seems to be quasi-reversible, mostly one-electron process (irreversible for 4) and most probably involve the central triphenylamine donor. The first reduction is a reversible two-electron process that may involve mostly diazine acceptor moiety and the adjacent $\pi$-system. The first oxidation/reduction potentials were found within the range of 0.61 to 0.69 and -2.02 to $-1.70 \mathrm{~V}$, respectively. Whereas the first oxidation is affected by the structural changes in $\mathbf{1}$ and 3-5 only negligibly, replacement of the peripheral diazine acceptor moieties and composition of the $\pi$-linker have significantly influenced the first reduction potentials in 1-5 and the corresponding electrochemical gap. A comparison of electrochemical behavior of chromophores 1, 3 and 5 allows evaluation of pyrimidine, quinoxaline and pyrazine moieties. The quinoxaline strongest withdrawing property is 
reflected by the most positive reduction potential and the lowest electrochemical gap being measured for 3. Replacement of 1,4-phenylene by 2,5-thienylene units in $\mathbf{1 / 2}$ and $\mathbf{3} / \mathbf{4}$ shifted the first reduction positively, while the gaps of $\mathbf{3}$ and $\mathbf{4}$ are almost identical. Thus, chromophores $\mathbf{3} / \mathbf{4}$ bearing the quinoxaline acceptor showed the lowest electrochemical gap as well as the most bathochromically shifted CT-bands, especially for 4 with 2,5-thienylene units. The same tendencies are observed for optical gap.

\section{$<$ Insert Table $4>$}

\section{Computational studies}

The spatial and electronic properties of chromophores 1-5 were investigated using Gaussian W09 package ${ }^{37}$ at the DFT level. The initial geometries of molecules 1-5 were estimated by PM3 method implemented in $\operatorname{ArgusLab}^{38}$ and these were subsequently optimized by DFT HCTH/6-311+G(d) method. The energies of the HOMO and LUMO, their differences and ground state dipole moments were calculated with the DFT B3LYP/6$311++\mathrm{G}(2 \mathrm{~d}, \mathrm{p})$ and are summarized in Table 4.

The calculated energies of the HOMO and LUMO of 1-5 were found within the same range as electrochemically measured values as can be seen on the energy level diagram shown in Figure 7. Moreover, both energies correlate tightly and, therefore, the used DFT method can be considered as a reasonable tool for description of the electronic properties of 1-5 (see the SI).

\section{$<$ Insert Figure 7 $>$}

Very similar trends as seen by the $\mathrm{CV}$ measurements can also be deduced from the DFT calculated energies of the HOMO and LUMO and their differences. The HOMO-LUMO gap can be reduced by replacement of the peripheral acceptor within the order of $\mathbf{5}>\mathbf{1}>\mathbf{2}>\mathbf{3}$ 
$>$ 4. Chromophores 3 and $\mathbf{4}$ bearing the most electron withdrawing quinoxaline moieties possess the lowest calculated gaps of 2.54 and $2.31 \mathrm{eV}$. The latter is further reduced by the presence of 2,5-thienylene instead of 1,4-phenylene $\pi$-linker. Positions of the frontier molecular orbitals in 1-5 are visualized in Figure 8. The HOMO is localized on the central amino donor and adjacent alternating positions of the triphenylamine core, whereas the LUMO is spread over the peripheral diazine acceptors and the adjacent $\pi$-linker. Both frontier orbitals are clearly separated which further confirms CT character of chromophores 1-5.

\section{$<$ Insert Figure $8>$}

Considering tripodal character and $\mathrm{C} 3$ symmetry group of each derivative 1-5, the LUMO is localized over one or two particular branche(s). The third branch is occupied by the $\mathrm{LUMO}+1$. The HOMO as well as the HOMO-1 remained on the central amino donor. This is a common feature and has already been observed earlier for tripodal molecules based on

triphenylamine. ${ }^{8 \mathrm{a}, 39}$ As expected, the calculated ground state dipole moments $\mu_{\mathrm{G}}$ are modest within the range of 0.08 to $4.79 \mathrm{D}$, which reflects tripodal character of chromophores 1-5.

\section{Conclusions}

In summary, we have successfully synthesized and characterized a series of triphenylaminebased tripodal molecules bearing peripheral diazine electron-attracting groups. The key step of their synthesis involves threefold Sonogashira cross-coupling reaction between tris(4ethynylphenyl)amine (donor central part) and the corresponding bromoarylvinyldiazine derivatives (peripheral acceptors). These compounds exhibit absorption in the blue region and are highly luminescent with significant Stokes shifts. For all the compounds a strong emission solvatochromism was observed in a variety of non-polar solvents. This finding supports formation of a very polar excited ICT state. These materials exhibit 2PA properties, measured by $2 \mathrm{PA}$ excited fluorescence spectroscopy within the spectral range of 700 to $1020 \mathrm{~nm}$. 2PA cross sections up to 427 GM were obtained. The HOMO and LUMO levels were estimated by 
cyclic voltametry measurements and DFT calculations. From all the performed measurements and calculations is apparent that derivatives 2 and 4, bearing 2,5-thienylene spacer, exhibit the highest 2PA cross section and the lowest HOMO-LUMO gap even if their solvatochromic ranges are lower than that of their 1,4-phenylene analogues. The pyrimidine and quinoxaline derivatives display similar 2PA cross sections but quinoxaline compounds showed lower HOMO-LUMO gap. In view of the current wide and general interest in novel 2PA absorbers, we believe that this joint study would be considered as a very useful guide in designing octopolar tripodal molecules bearing diazine peripheral acceptor groups.

\section{Experimental Section}

General. Compounds 6-8 were obtained according to reported procedures. ${ }^{22 a, 22 \mathrm{e}}$ Compound 11 was obtained from tris(4-iodophenyl)amine and TMSA by Sonogashira cross-coupling reaction according to reported procedure. ${ }^{8 \mathrm{a}, 40}$ All air- and moisture-sensitive reactions were carried out in flame-dried glassware subsequently cooled under nitrogen. NMR spectra were acquired at room temperature on a Bruker AC-300 spectrometer. Chemical shifts are given in parts per million relative to TMS $\left({ }^{1} \mathrm{H}, 0.0 \mathrm{ppm}\right)$ and $\mathrm{CDCl}_{3}\left({ }^{13} \mathrm{C}, 77.0 \mathrm{ppm}\right)$. Acidic impurities in $\mathrm{CDCl}_{3}$ were removed by treatment with anhydrous $\mathrm{K}_{2} \mathrm{CO}_{3}$. High resolution mass analyses were performed at the "Centre Régional de Mesures Physiques de l'Ouest" (CRMPO, University of Rennes1) using a Bruker MicroTOF-Q II apparatus. IR spectra were recorded on a Perkin-Elmer spectrum1000 FTIR using KBr plates. UV/vis spectra were recorded with a UVIKON xm SECOMAM spectrometer using standard $1 \mathrm{~cm}$ quartz cells. Fluorescence spectra were recorded using Spex FluoroMax-3 Jobin-Yvon Horiba apparatus. Compounds were excited at their absorption maxima (longest-wavelength absorption band) to record the emission spectra. The $\Phi_{\mathrm{F}}$ values were calculated using a well-known procedure using 9,10diphenylethynylanthracene in cyclohexane as standard. ${ }^{41}$ Stokes shifts were calculated by considering the lowest energetic absorption band. 
2PA measurement. The two-photon absorption (2PA) measurements were performed with femtosecond mode-locked laser pulse using a Ti: Sapphire laser (Coherent, Chameleon Ultra II: pulse duration: $\sim 140 \mathrm{fs}$; repetition rate: $80 \mathrm{MHz}$; wavelength range: 680-1040 nm). A relative $2 \mathrm{PEF}$ method ${ }^{42}$ was employed to measure the two-photon absorption cross-sections, $\delta$. The measurements of 2PA cross-sections were performed relative to reference molecules (r) such as fluorescein ${ }^{40,43}$ in water at $\mathrm{pH}=11$. The value of $\delta$ for a sample $(s)$ is given by:

$$
\delta_{S}=\frac{S_{S} \Phi_{r} \eta_{r} c_{r}}{S_{r} \Phi_{S} \eta_{S} c_{S}} \cdot \delta_{r}
$$

Where $S$ is the detected two-photon excited fluorescence integral area, $c$ the concentration of the chromophores, and $\Phi$ is the fluorescence quantum yield of the chromophores. $\eta$ is the collection efficiency of the experimental set-up and accounts for the wavelength dependence of the detectors and optics as well as the difference in refractive indices between the solvents in which the reference and sample compounds are dissolved. The measurements were conducted in a regime where the fluorescence signal showed a quadratic dependence on the intensity of the excitation beam, as expected for two-photon induced emission. For the calibration of the two-photon absorption spectra, the two-photon excited fluorescence signal of each compound was recorded at the same excitation wavelength as that used for standards (i.e. $\lambda_{\text {exc }}=782 \mathrm{~nm}$ for fluorescein). The laser intensity was in the range of $0.2-2 \times 10^{9} \mathrm{~W} / \mathrm{cm}^{2}$. The experimental error on the reported cross section is $15 \%$.

(E)-2-(2-(5-Bromothiophen-2-yl)vinyl)quinoxaline (9): 2-Methylquinoxaline (720 mg, 5.00 mmol) and 5-bromothiphene-2-carbaldehyde (955 $\mathrm{mg}, 5.00 \mathrm{mmol})$ were added to a solution of $\mathrm{NaOH}(25 \mathrm{~mL}, 5 \mathrm{M})$ with addition of approx. $100 \mathrm{mg}(0.25 \mathrm{mmol})$ of Aliquat ${ }^{\circledR} 336$. The resulting emulsion was heated up to reflux for $2 \mathrm{~h}$. After cooling to $25{ }^{\circ} \mathrm{C}$, the gummy-like precipitate was decanted, washed with water $(2 \times 25 \mathrm{~mL})$ and dried in vacuo. The crude 
product was crystallized from DCM/n-heptane to afford $860 \mathrm{mg}(54 \%)$ of 9 as a brownish yellow solid. Mp: $107-109{ }^{\circ} \mathrm{C}{ }^{1} \mathrm{H}-\mathrm{NMR}\left(300 \mathrm{MHz}, \mathrm{CDCl}_{3}\right): \delta(\mathrm{ppm}): 7.02-7.07$ (m, 3H), 7.73 (dquint, $\left.2 \mathrm{H},{ }^{3} J=6.9 \mathrm{~Hz},{ }^{4} J=1.5 \mathrm{~Hz}\right), 7.89\left(\mathrm{~d}, 1 \mathrm{H},{ }^{3} \mathrm{~J}=15.9 \mathrm{~Hz}\right), 8.04\left(\mathrm{dt}, 2 \mathrm{H},{ }^{3} J=6.9\right.$ $\left.\mathrm{Hz},{ }^{4} J=1.5 \mathrm{~Hz}\right), 8.92(\mathrm{~s}, 1 \mathrm{H}) .{ }^{13} \mathrm{C}-\mathrm{NMR}\left(75 \mathrm{MHz}, \mathrm{CDCl}_{3}\right): \delta(\mathrm{ppm}): 114.2(\mathrm{C}), 124.6(\mathrm{CH})$, $128.3(\mathrm{CH}), 129.1(\mathrm{CH}), 129.2(\mathrm{CH}), 129.3(2 \times \mathrm{CH}), 130.4(\mathrm{CH}), 131.0(\mathrm{CH}), 141.6(\mathrm{C})$, 142.5 (C), 143.2 (C), 144.5 (CH), 149.9 (C). IR (ATR) v: 3058, 1620, 1426, 1415, 947, 752 $\mathrm{cm}^{-1}$ HRMS (ESI/ASAP) $\mathrm{m} / \mathrm{z}$ calculated for $\mathrm{C}_{14} \mathrm{H}_{10}{ }^{79} \mathrm{BrN}_{2} \mathrm{~S}[\mathrm{M}+\mathrm{H}]^{+}$316.9748, found 316.9749 .

(E)-2-(4-Bromostyryl)pyrazine (10): 4-Bromobenzaldehyde (2.30 g, $12.5 \mathrm{mmol})$ and 2methylpyrazine $(1.17 \mathrm{~g}, 12.5 \mathrm{mmol})$ were dissolved in DMSO $(7.5 \mathrm{~mL})$. Powdered KOH $(2.75 \mathrm{~g}, 50.0 \mathrm{mmol})$ was added and the reaction mixture was stirred at room temperature for 8 $\mathrm{h}$ and then poured into $200 \mathrm{~mL}$ of water. The resulting precipitate was filtered and washed with water $(2 \times 150 \mathrm{~mL})$. The crude product was crystallized from $\mathrm{DCM} / n$-heptane to afford $10(2.01 \mathrm{~g}, 62 \%)$ as a brownish yellow solid. Mp: $110-112{ }^{\circ} \mathrm{C}{ }^{1} \mathrm{H}-\mathrm{NMR}\left(300 \mathrm{MHz}, \mathrm{CDCl}_{3}\right): \delta$ (ppm) : $7.14\left(\mathrm{~d}, 1 \mathrm{H},{ }^{3} \mathrm{~J}=16.2 \mathrm{~Hz}\right), 7.45\left(\mathrm{~d}, 2 \mathrm{H},{ }^{3} \mathrm{~J}=8.7 \mathrm{~Hz}\right), 7.52\left(\mathrm{~d}, 2 \mathrm{H},{ }^{3} \mathrm{~J}=3.4 \mathrm{~Hz}\right), 7.68$ $\left(\mathrm{d}, 1 \mathrm{H},{ }^{3} \mathrm{~J}=16.2 \mathrm{~Hz}\right), 8.42\left(\mathrm{~d}, 1 \mathrm{H},{ }^{3} J=2.4 \mathrm{~Hz}\right), 8.54\left(\mathrm{dd}, 1 \mathrm{H},{ }^{3} \mathrm{~J}=2.4 \mathrm{~Hz},{ }^{4} \mathrm{~J}=1.2 \mathrm{~Hz}\right), 8.62$ $\left(\mathrm{d}, 1 \mathrm{H},{ }^{4} J=1.2 \mathrm{~Hz}\right) \cdot{ }^{13} \mathrm{C}-\mathrm{NMR}\left(75 \mathrm{MHz}, \mathrm{CDCl}_{3}\right): \delta(\mathrm{ppm}): 123.0(\mathrm{C}), 124.7(\mathrm{CH}), 128.7$ $(\mathrm{CH}), 132.0(\mathrm{CH}), 133.9(\mathrm{CH}), 135.0(\mathrm{C}), 143.0(\mathrm{CH}), 143.8(\mathrm{CH}), 144.4(\mathrm{CH}), 150.9(\mathrm{C}) . \mathrm{IR}$ (ATR) v: 3048, 1487, 1403, 1009, $964 \mathrm{~cm}^{-1}$ HR-MS MALDI (matrix DHB): m/z calculated for $\mathrm{C}_{12} \mathrm{H}_{10}{ }^{79} \mathrm{BrN} 2[\mathrm{M}+2 \mathrm{H}]^{+} 262.0095$, found 262.0102.

General procedure for Sonogashira cross-coupling reaction: A mixture of $4,4^{6}, 4^{\circ}$ “tris(ethynyl)triphenylamine $\mathbf{1 1}(80 \mathrm{mg}, 0,25 \mathrm{mmol})$ and the corresponding bromo derivative (0.91 mmol) was dissolved in a mixture of 1,4-dioxane $(20 \mathrm{~mL})$ and diisopropylamine $(5 \mathrm{~mL})$. Nitrogen was bubbled through the mixture for $10 \mathrm{~min}$ whereupon $\left[\mathrm{Pd}\left(\mathrm{PPh}_{3}\right)_{4}\right](29 \mathrm{mg}, 0.025$ 
mmol, $10 \%)$ and $\mathrm{CuI}(9 \mathrm{mg}, 0.05 \mathrm{mmol}, 20 \%)$ were added and the reaction mixture was stirred $92{ }^{\circ} \mathrm{C}$ for $12 \mathrm{~h}$. The reaction was quenched with water $(50 \mathrm{~mL})$, extracted with DCM $(2 \times 100 \mathrm{~mL})$ and the combined organic extracts were dried over $\mathrm{MgSO}_{4}$ and the solvents were evaporated in vacuo. The crude product was purified by column chromatography with the indicated stationary phase and the solvent system.

Tris-\{4-[4-((E)-2-pyrimidin-4-yl-vinyl)-phenylethynyl]-phenyl\}-amine (1): The title compound was obtained from $\mathbf{6}$ following the general procedure for Sonogashira crosscoupling reaction and was purified by column chromatography $\left(\mathrm{Al}_{2} \mathrm{O}_{3}\right.$, EtOAc). Yellow solid (81 mg, $38 \%$. Mp: $190{ }^{\circ} \mathrm{C}$ (dec.). ${ }^{1} \mathrm{H}-\mathrm{NMR}\left(300 \mathrm{MHz}, \mathrm{CD}_{2} \mathrm{Cl}_{2}\right): \delta(\mathrm{ppm}): 7.12-7.18$ (m, 9H), $7.38\left(\mathrm{dd}, 3 \mathrm{H},{ }^{4} J=1.2 \mathrm{~Hz},{ }^{3} J=5.4 \mathrm{~Hz}\right), 7.52\left(\mathrm{~d}, 6 \mathrm{H},{ }^{3} J=9.0 \mathrm{~Hz}\right), 7.59\left(\mathrm{~d}, 6 \mathrm{H},{ }^{3} J=8.4\right.$ Hz), $7.67\left(\mathrm{~d}, 6 \mathrm{H},{ }^{3} \mathrm{~J}=8.4 \mathrm{~Hz}\right), 7.94\left(\mathrm{~d}, 3 \mathrm{H},{ }^{3} \mathrm{~J}=15.9 \mathrm{~Hz}\right), 8.71\left(\mathrm{~d}, 3 \mathrm{H},{ }^{3} \mathrm{~J}=5.4 \mathrm{~Hz}\right), 9.16(\mathrm{~d}$, $\left.3 \mathrm{H},{ }^{4} \mathrm{~J}=1.2 \mathrm{~Hz}\right) .{ }^{13} \mathrm{C}-\mathrm{NMR}\left(75 \mathrm{MHz}, \mathrm{CD}_{2} \mathrm{Cl}_{2}\right): \delta(\mathrm{ppm}): 89.5(\mathrm{C}), 91.5(\mathrm{C}), 118.2(\mathrm{C}), 119.2$ (CH), $124.5(\mathrm{CH}), 124.7(\mathrm{C}), 126.9(\mathrm{CH}), 128.0(\mathrm{CH}), 132.3(\mathrm{CH}), 133.2(\mathrm{CH}), 135.9(\mathrm{C})$, $136.5(\mathrm{CH}), 147.3(\mathrm{C}), 157.9(\mathrm{CH}), 159.2(\mathrm{CH}), 162.3(\mathrm{C}) . \mathrm{IR}(\mathrm{ATR})$ v: $2208(\mathrm{C} \equiv \mathrm{C}), 1573$, 1512, 1090, $833 \mathrm{~cm}^{-1}$ HRMS MALDI (matrix DHB) m/z calculated for $\mathrm{C}_{60} \mathrm{H}_{39} \mathrm{~N}_{7}\left[\mathrm{M}^{+}\right]$ 857.3267, found 857.3270 .

Tris-\{4-[5-((E)-2-pyrimidin-4-yl-vinyl)-thiophen-2-ylethynyl]-phenyl\}-amine (2): The title compound was obtained from 7 following the general procedure for Sonogashira crosscoupling reaction and was purified by column chromatography $\left(\mathrm{Al}_{2} \mathrm{O}_{3}\right.$, EtOAc). Reddishorange solid (61 mg, $28 \%$ ). Mp: $110{ }^{\circ} \mathrm{C}(\mathrm{dec}) .{ }^{1} \mathrm{H}-\mathrm{NMR}\left(300 \mathrm{MHz}, \mathrm{CDCl}_{3}\right): \delta(\mathrm{ppm}): 6.83$ $\left(\mathrm{d}, 3 \mathrm{H},{ }^{3} J=15.9, \mathrm{~Hz}\right), 7.11\left(\mathrm{~d}, 6 \mathrm{H},{ }^{3} J=8.7 \mathrm{~Hz}\right), 7.19\left(\mathrm{~d}, 3 \mathrm{H},{ }^{3} J=3.4 \mathrm{~Hz}\right), 7.20\left(\mathrm{~d}, 3 \mathrm{H},{ }^{3} J=\right.$ 3.4 Hz), 7.27-7.26 (m, 3H), 7.46 (d, 6H, $\left.{ }^{3} J=8.7 \mathrm{~Hz}\right), 8.01$ (d, 3H, $\left.{ }^{3} J=15.9, \mathrm{~Hz}\right), 8.68$ (d, 3H, $\left.{ }^{3} J=5.1 \mathrm{~Hz}\right), 9.16(\mathrm{~s}, 3 \mathrm{H}) .{ }^{13} \mathrm{C}-\mathrm{NMR}\left(75 \mathrm{MHz}, \mathrm{CDCl}_{3}\right): \delta(\mathrm{ppm}): 82.8(\mathrm{C}), 95.4(\mathrm{C}), 117.4$ (C), $118.8(\mathrm{CH}), 124.1(\mathrm{CH}), 125.1(\mathrm{CH}), 125.2(\mathrm{C}), 129.6(\mathrm{CH}), 129.9(\mathrm{CH}), 132.7(\mathrm{CH})$, 
$132.8(\mathrm{CH}), 142.1(\mathrm{C}), 146.9(\mathrm{C}), 157.5(\mathrm{CH}), 158.8(\mathrm{CH}), 161.5(\mathrm{C})$. IR (ATR) v: 3035, 2203, 1590, 1513, 1496, 1314, 1288, 818, $756 \mathrm{~cm}^{-1}$ HRMS MALDI (matrix DHB) m/z calculated for $\mathrm{C}_{54} \mathrm{H}_{33} \mathrm{~N}_{7} \mathrm{~S}_{3}[\mathrm{M}+\mathrm{H}]^{+}$876.2038, found 876.2051.

Tris-\{4-[4-((E)-2-quinoxalin-2-yl-vinyl)-phenylethynyl]-phenyl\}-amine $\quad(3): \quad$ The title compound was obtained from $\mathbf{8}$ following the general procedure for Sonogashira crosscoupling reaction and was purified by column chromatography $\left(\mathrm{Al}_{2} \mathrm{O}_{3}, \mathrm{EtOAc}\right)$. Orange solid (70 mg, $28 \%$ ). Mp: $190{ }^{\circ} \mathrm{C}(\mathrm{dec}) .{ }^{1} \mathrm{H}-\mathrm{NMR}\left(300 \mathrm{MHz}, \mathrm{CDCl}_{3}\right): \delta(\mathrm{ppm}): 7,11\left(\mathrm{~d}, 6 \mathrm{H},{ }^{3} J=\right.$ $8.7 \mathrm{~Hz}), 7.42\left(\mathrm{~d}, 3 \mathrm{H},{ }^{3} \mathrm{~J}=16.2 \mathrm{~Hz}\right), 7.57\left(\mathrm{~d}, 6 \mathrm{H},{ }^{3} \mathrm{~J}=8.7 \mathrm{~Hz}\right), 7.58\left(\mathrm{~d}, 6 \mathrm{H},{ }^{3} J=8.4 \mathrm{~Hz}\right), 7.68$ (d, 6H, $\left.{ }^{3} J=8.4 \mathrm{~Hz}\right), 7.80-7.72(\mathrm{~m}, 6 \mathrm{H}), 7.88\left(\mathrm{~d}, 3 \mathrm{H},{ }^{3} J=16.2 \mathrm{~Hz}\right), 8.10-8.06(\mathrm{~m}, 6 \mathrm{H}), 9.05(\mathrm{~s}$, 3H). ${ }^{13} \mathrm{C}-\mathrm{NMR}\left(75 \mathrm{MHz}, \mathrm{CDCl}_{3}\right): \delta(\mathrm{ppm}): 89.4(\mathrm{C}), 91.1(\mathrm{C}), 117.9(\mathrm{C}), 124.1(\mathrm{CH}), 124.2$ (C), $125.9(\mathrm{CH}), 127.4(\mathrm{CH}), 129.2(\mathrm{CH}), 129.4(\mathrm{CH}), 129.7(\mathrm{CH}), 130.4(\mathrm{CH}), 132.0(\mathrm{CH})$, $132.9(\mathrm{CH}), 135.6(\mathrm{CH}), 135.7(\mathrm{C}), 141.6(\mathrm{C}), 142.5(\mathrm{C}), 144.5(\mathrm{CH}), 146.8(\mathrm{C}), 150.4(\mathrm{C})$. IR (ATR) v: 3035, 2207 (C巨C), 1589, 1512, 1493, 1313, 1288, 818, $757 \mathrm{~cm}^{-1}$ HRMS MALDI (matrix DHB) $\mathrm{m} / \mathrm{z}$ calculated for $\mathrm{C}_{72} \mathrm{H}_{45} \mathrm{~N}_{7}[\mathrm{M}+\mathrm{H}]^{+}$1008.3815, found 1008.3782.

Tris-\{4-[5-((E)-2-quinoxalin-2-yl-vinyl)-thiophen-2-ylethynyl]-phenyl\}-amine (4): The title compound was obtained from 9 following the general procedure for Sonogashira crosscoupling reaction and was purified by column chromatography $\left(\mathrm{SiO}_{2}\right.$, Petroleum Ether/EtOAc/NEt $3,13 / 6 / 1)$ followed by crystallization from MeOH. Red solid (155 mg, 60 \%). Mp: $158-160{ }^{\circ} \mathrm{C} .{ }^{1} \mathrm{H}-\mathrm{NMR}\left(300 \mathrm{MHz}, \mathrm{CDCl}_{3}\right): \delta(\mathrm{ppm}): 7.20\left(\mathrm{~d}, 6 \mathrm{H},{ }^{3} \mathrm{~J}=8.7 \mathrm{~Hz}\right), 7.27$ $\left(\mathrm{d}, 3 \mathrm{H},{ }^{3} J=3.4 \mathrm{~Hz}\right), 7.30\left(\mathrm{~d}, 3 \mathrm{H},{ }^{3} J=3.4 \mathrm{~Hz}\right), 7.35\left(\mathrm{~d}, 3 \mathrm{H},{ }^{3} J=15.9, \mathrm{~Hz}\right), 7.56\left(\mathrm{~d}, 6 \mathrm{H},{ }^{3} J=\right.$ 8.7 Hz), 7.88-7.77 (m, 6H), $8.05\left(\mathrm{~d}, 3 \mathrm{H},{ }^{3} \mathrm{~J}=15.9, \mathrm{~Hz}\right), 8.17-8.14(\mathrm{~m}, 6 \mathrm{H}), 9.05(\mathrm{~s}, 3 \mathrm{H}) .{ }^{13} \mathrm{C}-$ NMR (75 MHz, $\left.\mathrm{CDCl}_{3}\right): \delta(\mathrm{ppm}): 82.9(\mathrm{C}), 95.2(\mathrm{C}), 117.5(\mathrm{C}), 124.1(\mathrm{CH}), 124.6(\mathrm{C}), 124.9$ (CH), $128.6(\mathrm{CH}), 129.0(\mathrm{CH}), 129.1(\mathrm{CH}), 129.2(\mathrm{CH}), 129.3(\mathrm{CH}), 130.4(\mathrm{CH}), 132.7(\mathrm{CH})$, $132.8(\mathrm{CH}), 141.5(\mathrm{C}), 142.5(\mathrm{C}), 142.7(\mathrm{C}), 144.5(\mathrm{CH}), 146.8(\mathrm{C}), 150.0(\mathrm{C})$. IR (ATR) v: 
3028, $2190(\mathrm{C} \equiv \mathrm{C}), 1618,1593,1523,1497,1285,949,754 \mathrm{~cm}^{-1}$ HRMS (ESI/ASAP) m/z calculated for $\mathrm{C}_{66} \mathrm{H}_{40} \mathrm{~N}_{7} \mathrm{~S}_{3}[\mathrm{M}+\mathrm{H}]^{+}$1026.2502, found 1026.2501.

Tris-\{4-[4-((E)-2-pyrazin-2-yl-vinyl)-phenylethynyl]-phenyl\}-amine $\quad$ (5): $\quad$ The title compound was obtained from $\mathbf{1 0}$ following the general procedure for Sonogashira crosscoupling reaction and was purified by column chromatography $\left(\mathrm{SiO}_{2}, \mathrm{EtOAc} / \mathrm{NEt}_{3}, 19 / 1\right)$ followed by crystallization from $\mathrm{MeOH}$. Yellow solid (50 mg, $23 \%$ ). Mp: $110{ }^{\circ} \mathrm{C}(\mathrm{dec}) .{ }^{1} \mathrm{H}-$ NMR (300 MHz, $\left.\mathrm{CDCl}_{3}\right): \delta(\mathrm{ppm}): 7.10\left(\mathrm{~d}, 6 \mathrm{H},{ }^{3} J=8.4 \mathrm{~Hz}\right), 7.17\left(\mathrm{~d}, 3 \mathrm{H},{ }^{3} J=16.2 \mathrm{~Hz}\right), 7.46$ $\left(\mathrm{d}, 6 \mathrm{H},{ }^{3} \mathrm{~J}=8.4 \mathrm{~Hz}\right), 7.53\left(\mathrm{~d}, 6 \mathrm{H},{ }^{3} J=8.4 \mathrm{~Hz}\right), 7.58\left(\mathrm{~d}, 6 \mathrm{H},{ }^{3} \mathrm{~J}=8.4 \mathrm{~Hz}\right), 7.75\left(\mathrm{~d}, 3 \mathrm{H},{ }^{3} J=\right.$ 15.9, Hz), 8.42 (broad s, 3H), 8.56 (broad s, 3H), 8.65 (broad s, 3H). ${ }^{13} \mathrm{C}-\mathrm{NMR}(75 \mathrm{MHz}$, $\left.\mathrm{CDCl}_{3}\right): \delta(\mathrm{ppm}): 82.3(\mathrm{C}), 89.9(\mathrm{C}), 117.9(\mathrm{C}), 124.1(\mathrm{CH}), 124.7(\mathrm{CH}), 127.1(\mathrm{CH}), 131.6$ (C), $131.9(\mathrm{CH}), 132.0(\mathrm{CH}), 132.9(\mathrm{CH}), 134.4(\mathrm{CH}), 135.8(\mathrm{C}), 143.9(\mathrm{CH}), 144.4(\mathrm{CH})$, 146.8 (C), 151.1 (C). IR (ATR) v: 3035, 2207 (C $=C), 1589,1512,1493,1313,1288,818,757$ $\mathrm{cm}^{-1}$ HRMS (ESI/ASAP) $\mathrm{m} / \mathrm{z}$ calculated for $\mathrm{C}_{60} \mathrm{H}_{40} \mathrm{~N}_{7}[\mathrm{M}+\mathrm{H}]^{+} 858.3340$, found 858.3337 .

Acknowledgement: D. C., O. P. and F. B. are indebted to the Czech Science Foundation (1301061S). N. C. gratefully acknowledges Dr. F. Gloaguen and UMR CNRS 6521 for their support.

Supporting Information Available: copies of ${ }^{1} \mathrm{H}$ NMR and ${ }^{13} \mathrm{C}$ NMR spectra for all compounds, plots of emission maxima versus $\mathrm{E}_{\mathrm{T}}(30)$, representative $\mathrm{CV}$ diagram and correlations. This material is available free of charge via the internet. 


\section{Chart 1.}

Scheme 1. Synthesis of bromo-substituted intermediates 6-9.

Scheme 2. Synthesis of compound 10.

Scheme 3. Final Sonogashira cross-coupling $\left(\operatorname{Pd}\left(\mathrm{PPh}_{3}\right)_{4}, \mathrm{CuI}, 1,4-\right.$ dioxane, $i \operatorname{Pr}_{2} \mathrm{HN}, 92$ ${ }^{\circ} \mathrm{C}$, 12h).

Figure 1: Normalized UV/vis (solid lines) and emission spectra (dashed lines) of compounds 1 and 4.

Figure 2. Normalized emission of compound 2 in different aprotic solvents.

Figure 3. Fluorescence color changes for 3 in various solvents (from left to right: hexane, toluene, dioxane, THF and dichoromethane. Picture was taken in the dark upon irradiation with a hand-held UV $\operatorname{lamp}\left(\lambda_{\mathrm{em}}=366 \mathrm{~nm}\right)$.

Figure 4: Color change of a DCM solution $\left(c=10^{-1} \mathrm{M}\right)$ of 4 in the presence of TFA.

Figure 5: UV/vis spectra of 2 in DCM $\left(c=610^{-6} \mathrm{M}\right)$ with and without addition of TFA (c $\left.=10^{-1} \mathrm{M}\right)$.

Figure 6: Two-photon absorption spectra of the chromophores 1-5 in dichloromethane.

Figure 7: Energy level diagram showing electrochemical (black) and DFT calculated (red) HOMO and LUMO levels of 1-5.

Figure 8: HOMO/HOMO-1 (red) and LUMO/LUMO+1 (blue) localization in 4 and HOMO/LUMO mix in 1-3 and 5.

Table 1. UV/Vis and photoluminescence (PL) data in $\mathrm{CH}_{2} \mathrm{Cl}_{2}$.

Table 2. Emission solvatochromism of diazine derivatives 1-5 in various aprotic solvents.

Table 3. Two-photon absorption photophysical properties of compounds 1-5 in dichloromethane. 
Table 4. Electrochemical, ${ }^{a}$ and DFT calculated data as well as optical gap for chromophores 1-5.

${ }^{1}$ S. Allard, M. Forster, B. Souharce, H. Thiem, U. Scherf Angew. Chem. Int. Ed. 2008, 47, 4070-4098.

2 a) Y. Ohmiri, Laser Photonics Rev. 2009, 4, 300-310. b) K. Walzer, B. Maennig, M. Pfeiffer, K. Leo, Chem. Rev. 2007, 107, 1233-1271.

${ }^{3}$ a) Y. Wu, W. Zhu, Chem. Soc. Rev. 2013, 42, 2039-2058. b) C. Duan, K. Zhang, C. Zhong, F. Huang, Y. Cao, Chem. Soc. Rev. 2013, 42, 9071-9104. c) C. Duan, F. Huang, Y. Cao, J. Mater. Chem. 2012, 22, 10416-10434. d) J. N. Clifford, E. Martínez-Ferrero, A. Viterisi, E. Palomares, Chem. Soc. Rev. 2011, 40, 1635-1646. e) A. W. Hains, Z. Liang, M. A. Woodhouse, B. A. Gregg, Chem. Rev. 2010, 110, 6689-6735.

${ }^{4}$ a) S. Barlow, S. R. Marder, In: Functional Organic Materials; Müller, TJJ, Bunz UHF, Eds.; Wiley-VCH: Weinheim, Germany, 2007; p. 393-437. b) R. R. Tykwinski, U. Gubler, R. E. Martin, F. Diederich, C. Bosshard, P. Günter, P. J. Phys. Chem. B 1998, 102, 4451-4465.

${ }^{5}$ F. Bureš, F. RSC Adv. 2014, 4, 58826-58851.

${ }^{6}$ a) Z. Hu, X. D. Li, W. J. Zhang, A. H. Liang, D. D. Ye, Z. T. Liu, J. Liu, Y. J. Liu, J. F. Fang, $R S C A d v$. 2014, 4, 5591-5597. b) S. Ren, D. Zeng, H. Zhong, Y. Wang, S. Qian, Q. Fang, J. Phys. Chem. B 2010, 114, 10374-10383. c) J. Cremer, P. Baüerle, P. J. Mater. Chem. 2006, 16, 874-884. d) B. Li, J. Li, Y. Fu, Z. Bo, J. Am. Chem. Soc. 2004, 126, 3430-3431. e) A. L. Kanibolotsky, R.Berridge, P. J. Skabara, I. F. Perepichka, D. D. C. Bradley, M. Koeberg, J. Am. Chem. Soc. 2004, 126, 13695-13702.

${ }^{7}$ a) H. Detert, M. Lehmann, H. Meier, Materials 2010, 3, 3218-3330. b) F. Terenziani, C. Le Droumaguet, C. Katan, O. Mongin, M. Blanchard-Desce ChemPhysChem 2007, 8, 723-734. 
c) S. Kato, T. Matsumoto, M. Shigeiwa, H. Gorohmaru, S. Maeda, T. Ishi-i, S. Mataka, Chem.

Eur. J. 2006, 12, 2303-2317. d) C. Katan, F. Terenziani, O. Mongin, M. H. V Werts, L. Porres, T. Pons, J. Mertz, S. Tretiak, M. Blanchard-Desce, J. Phys. Chem. A 2005, 109, 30243037.

${ }^{8}$ a) P. Hrobárik, V. Hrobáriková, I. Sigmundová, P. Zahradník, M. Fakis, I. Polyzos, P. Persephonis, J. Org. Chem. 2011, 76, 8726-8736. b) L. Porres, O. Mongin, C. Katan, M. Charlot, T. Pons, J. Mertz, M. Blanchard-Desce Org. Lett. 2004, 6, 47-50. c) E. Piovesan, L. De Boni, E. Ishow, C. R. Mendonca, Chem. Phys. Lett. 2010, 498, 277-280. d) Y. Jiang, Y. Wang, J. Hua, J. Tang, B. Li, S. Qian, H. Tian, Chem Commun. 2010, 46, 4689-4691.

${ }^{9}$ a) B. Dumat, G. Bordeau, E. Faurel-Paul, F. Mahuteau-Betzer, N. Saettel, G. Metge, C. Fiorini-Debuisschert, F. Charra, M.-P. Teulade-Fichou, J. Am. Chem. Soc. 2013, 135, 1269712706. b) C. Le Droumaguet, A. Sourdon, E. Genin, O. Mongin, M. Blanchard-Desce Chem. Asian J. 2013, 8, 2984-3001.c) B. Dumat, G. Bordeau, A. I. Aranda, F.Mahuteau-Betzer, Y. El Harfouch, G. Metge, F. Charra, C. Fiorini-Debuisschert, M.-P. Teulade-Fichou, Org. Biomol. Chem. 2012, 10, 6054-6061. d) R. Lartia, C. Allain, G. Bordeau, F. Schmidt, C. Fiorini-Debuisschert, F. Charra, M.-P. Teulade-Fichou, J. Org. Chem. 2008, 73, 1732-1744.

10 a) S. Yao, K. D. Belfield, Eur. J. Org. Chem. 2012, 3199-3217. b) G. Wang, K.-Y. Pu, X. Zhang, K. Li, L. Wang, L. Cai, D. Ding, Y.-H. Lai, B. Liu, Chem. Mater. 2011, 23, 44284434. c) G. Wang, X. Zhang, J. Geng, K. Li, D. Ding, K.-Y. Pu, L. Cai, Y.-H. Lai, B. Liu, Chem. Eur. J. 2012, 18, 9705-9713. d) H. M. Kim, B. R. Cho, Acc. Chem. Res. 2009, 42, 863872.

11 a) O. Mongin, M. Sankar, M. Charlot, Y. Mir, M. Blanchard-Desce, Tetrahedron Lett. 2013, 54, 6474-6478. b) F. Hammerer, S. Achelle, P. Baldeck, P. Maillard, M.-P. Teulade- 
Fichou, J. Phys. Chem. A 2011, 115, 6503-6508. c) S. Achelle, P. Couleaud, P. Baldeck, M.-

P. Teulade-Fichou, P. Maillard, Eur. J. Org. Chem. 2011, 7, 1271-1279.

12 a) D. A. Parthenopoulos, P. M. Rentzepis, Science 1989, 245, 843-845. b) J. H. Strickler, W. W. Webb, Opt. Lett. 1991, 16, 1780-1782. c) B. H. Cumpston, S. P. Ananthavel, S. Barlow, D. L. Dyer, J. E. Ehrlich, L. L. Erskine, A. A. Heikal, S. M. Kuebler, I.-Y, Sandy Lee, D. McCord-Maughon, J. Qin, H. Röckel, M. Rumi, X.-L. Wu, S. R. Marder, J. W. Perry, Nature 1999, 398, 51-54.

${ }^{13}$ a) M. Jin, J. Xie, J.-P. Malval, A. Spangenberg, O. Soppera, D.-L. Versace, T. Leclerc, H. Pan, D. Wan, H. Pu, P. Baldeck, O. Poizat, S. Knopf, J. Mater. Chem. C 2014, 2, 7201-7215. b) W. Zhou, S. M. Kuebler, K. L. Braun, T. Yu, J. K. Cammack, C. K. Ober, J. W. Perry, S. R. Marder, Science 2002, 296, 1106-1109. c) L. Kelemen, P. Ormos, G. Vizsnyiczai, J. Eur. Opt. Soc.-Rapid 2011, 6, 11029.

${ }^{14}$ a) Y. Qian, M. Luo, Dyes Pigm. 2014, 101, 240-246. b) L. Guo, K. F. Li, M. S. Wong, K. W. Cheah, Chem. Commun. 2013, 49, 3579-3599. c) T.-C. Lin, Q. Zheng, C.-Y. Chen, G. S. He, W.-J. Huang, A. I. Ryasnyanskiy, P. N. Prasad, Chem. Commun. 2008, 389-391.

15 a) T. Kengthanomma, P. Thamyongkit, J. Gasiorowski, A. M. Ramil, N. S. Sariciftci, J. Mater. Chem. A 2013, 1, 10524-10531. b) J. Zhang, J. Yu, C. He, D. Deng, Z.-G. Zhang, M. Zhang, Z. Li, Y. Li, Org. Electron. 2012, 13, 166-172. c) J. Zhang, D. Deng, C. He, Y. He, M. Zhang, Z.-G. Zhang, Z. Zhang, Y. Li, Chem. Mater. 2011, 23, 817-822. d) J. Jia, Y. Zhang, P. Xue, P. Zhang, X. Zhao, B. Liu, R. Lu, Dyes Pigm. 2013, 96, 407-413. e) Z. Ning, H. Tian, Chem. Commun. 2009, 5483-5495.

${ }^{16}$ E. Gagnon, T. Maris, J. D. Wuest, Org. Lett. 2010, 12, 404-407.

${ }^{17}$ K. Melanova, D. Cvejn, F. Bureš, V. Zima, J. Svoboda, L. Beneš, T. Mikysek, O. Pytela, P. Knotek, Dalton Trans. 2014, 43, 10462-10470. b) J. F. Eubank, F. Nouar, R.Luebke, A. J. 
Cairns, L. Wojtas, M. Alkordi, T. Bosquet, M. R. Hight, J. Eckert, J. P. Embs, P. A. Georgiev,

M. Eddaoudi, Angew. Chem. Int. Ed. 2012, 51, 10099-10103. c) D. Zhao, D. Yuan, D. Sun, H.-C. Zhou, J. Am. Chem. Soc. 2009, 131, 9186-9188.

18 a) N. Niammont, N. Kimpitak, G. Tumcharern, P. Rashatasakhon, M. Sukwattanasinitt RSC Adv. 2013, 3, 25215-25220. b) N. Niammont, W. Siripornnoppakhun, P. Rashatasakhon, M. Sukwattanasinitt, Org. Lett. 2009, 11, 2768-2771. c) S. Körsten, G. J. Mohr, Chem. Eur. J. 2011, 17, 969-975. d) N. Niammont, N. Kimpitak, K. Wongravee, P. Rashatasakhon, K. K. Baldridge, J. S. Siegel, M. Sukwattanasinitt, Chem. Commun. 2013, 49, 780-782. e) Z. Ning, Z. Chen, Q. Zhang, Y. Yan, S. Qian, Y. Cao, H. Tian, Adv. Funct. Mater. 2007, 17, 37993807.

19 a) S. Achelle, C. Baudequin C. In Targets in Heterocyclic Systems; Attanasi, O. A., Spinelli, D. 2013; Vol. 17, 1-34. b) S. Achelle, C. Baudequin, N. Plé, Dyes Pigm. 2013, 98, 575-600. c) S. Achelle, N. Plé, Curr. Org. Synth. 2012, 9, 163-187.

${ }^{20}$ See for example: a) P. Savel, H. Akdas-Kilig, J.-P. Malval, A. Spangenberg, T. Roisnel, J.L. Fillaut, J. Mater. Chem. C 2014, 2, 295-305. b) A. Wang, L. Long, S. Meng, X. Li, W. Zhao, Y. Song, M. P. Cifuentes, M. G. Humphrey, C. Zhang, Org. Biomol. Chem. 2013, 11, 4250-4257. c) D. Chen, C. Zhong, X. Dong, Z. Liu, J. Qin, J. Mater. Chem. 2012, 22, 43434348. d) L. Li, J. Ge, H. Wu, Q.-H.. Xu, S. Q. Yao, J. Am. Chem. Soc. 2012, 134, 1215712167.

${ }^{21}$ a) S. Achelle, J. Rodríguez-López F. Robin-le Guen, J. Org. Chem. 2014, 79, 7564-7571. b) C. Denneval, S. Achelle, C. Baudequin, F. Robin-le Guen Dyes Pigm. 2014, 110, 49-55. c) C. Hadad, S. Achelle, J. C. García-Martínez, J. Rodríguez-López J. Org. Chem. 2011, 76, 38373845. d) S. Achelle, I. Nouira, B. Pfaffinger, Y. Ramondenc, N. Plé, J. Rodríguez-López, J. Org. Chem. 2009, 74, 3711-3717. 
${ }^{22}$ a) S. Achelle, A. Barsella, B. Caro, F. Robin-le Guen. RSC Adv. 2015, 5, 39218-39227. b)

S. Achelle, S. Kahlal, A. Barsella, J.-Y. Saillard, X. Che, J. Vallet, F. Bureš B. Caro, F. Robin-le Guen Dyes Pigm. 2015, 113, 562-570. c) S. Achelle, S. Kahlal, J.-Y. Saillard, N. Cabon, B. Caro, F. Robin-le Guen, Tetrahedron 2014, 70, 2804-2815. d) S. Achelle, J.-P. Malval, S. Aloise, A. Barsella, A. Spangenberg, L. Mager, H. Akdas-Kilig, J.-L. Fillaut, B. Caro, F. Robin-le Guen ChemPhysChem 2013, 14, 2725-2736. e) S. Achelle, A.; Barsella, C. Baudequin, B. Caro, F. Robin-le Guen J. Org. Chem. 2012, 77, 4087-4096. f) F. Bureš H. Čermáková, J. Kulhánek, M. Ludwig, W. Kuznik, I. Kityk, T. Mikysek, A. Růžička, Eur. J. Org. Chem. 2012, 529-538.

${ }^{23}$ a) J.-P. Malval, S. Achelle, L. Bodiou, A. Spangenberg, L. Chia Gomez, O. Soppera, F. Robin-le Guen J. Mater. Chem. C 2014, 2, 7969-7880. b) C. Denneval, O. Moldovan, C. Baudequin S. Achelle, P. Baldeck, N. Plé, M. Darabantu, Y. Ramondenc, Eur. J. Org. Chem. 2013, 5591-5692.

24 F.-A. Martin, C. Baudequin, C. Fiol-Petit, M. Darabantu, Y. Ramondenc, N. Plé, Tetrahedron 2014, 70, 2546-2555.

25 J.-J. Vanden Eynde, L. Pascal Y. Van Haverbeke, P. Dubois, Synth. Commun. 2001, 31, $3167-3173$.

${ }^{26}$ H. Pan, X. Gao, Y. Zhang, P. N. Prasad, B. Reinhardt, R. Kanna, Chem. Mater. 1995, 7, 816-821.

${ }^{27}$ T. Szotkowski, F. Bureš, O. Pytela, J. Kulhánek, Z. Trávníček, J. Heterocycl. Chem. 2006, $43,1583-1589$.

${ }^{28}$ J. Kulhánek, F. Bureš, J. Opršal, W. Kuznik, T. Mikysek, A. Růžička, Asian J. Org. Chem. 2013, 2, 422-431. 
${ }^{29}$ a) V. Zilinskaite, D. Gudeika, J. V. Grazulevicius, D. Volyniuk, G. Buika, V. Jankauskas, G. Juska, M. Rutkis, A. Tokmakov, Dyes Pigm. 2015, 113, 38-46. b) M. Koening, G. Bottari, G. Brancato, V. Barone, D. M. Guldi, T. Torres, Chem. Sci. 2013, 4, 2502-2511. c) M. Alfonso, A. Espinosa, A. Tárraga, P. Molina, ChemistryOpen 2014, 3, 242-249.

${ }^{30}$ a) V. Schmitt, S. Moschel, H. Detert, H. Eur. J. Org. Chem. 2013, 5655-5669. b) A. S. Cornec, C. Baudequin, C. Fiol-Petit, N. Plé, G. Dupas, Y. Ramondenc, Y. Eur. J. Org. Chem. 2013, 1908-1915. c) C. Wink, H. Detert, J. Phys. Org. Chem. 2013, 26, 137-143.

31 a) J.-P. Cerón-Carrasco, D. Jacquemin, C. Laurence, A. Planchat, C. Reichardt, K. Sraïdi, J. Phys. Org. Chem. 2014, 27, 512-518. b) C. Reichardt, Chem. Rev. 1994, 94, 2319-2358.

${ }^{32}$ In ref $9 b$, compound $\mathbf{1 6 b}$ (analogue to compound $\mathbf{3}$ in the present article): in toluene $\lambda_{\text {em }}=$ $474 \mathrm{~nm}$ and in $\mathrm{CH}_{2} \mathrm{Cl}_{2} \lambda_{\mathrm{em}}=630 \mathrm{~nm}$. Compound 17 (analogue to compound 4 in the present article): in toluene $\lambda_{\text {em }}=523 \mathrm{~nm}$ and in $\mathrm{CH}_{2} \mathrm{Cl}_{2} \lambda_{\mathrm{em}}=610 \mathrm{~nm}$.

${ }^{33}$ S. Achelle F. Robin-le Guen, Tetrahedron Lett. 2013, 54, 4491-4496.

34 a) D. Beljonne, W. Wenseleers, E. Zojer, Z. Shuai, H. Vogel, S. J. K. Pond, J. W. Perry, S. R. Marder, J. L. Brédas, Adv. Funct. Mater. 2002, 12, 631-641. b) A. S. Davidov, Theory of molecular excitons; Plenum Press: New York, 1971.

${ }^{35}$ F. Terenziani, C. Katan, E. Badaeva, S. Tretiak, M. Blanchard-Desce, Adv. Mater. 2008, 20, 4641-4678.

36 a) S.-J. Chung, K.-S. Kim, T.-C. Lin, G. S. He, J. Swiatkiewicz, P. N. Prasad, J. Phys. Chem. B 1999, 103, 10741-10745. b) A. Bhaskar, G. Ramakrishna, Z. Lu, R. Twieg, J. M. Hales, D. J. Hagan, E. Van Stryland, T. Goodson, J. Am. Chem. Soc. 2006, 128, 11840-11849. c) B. R. Cho, K. H. Son, S. H. Lee, Y.-S. Song, Y.-K. Lee, S.-J. Jeon, J. H. Choi, H. Lee, M. Cho,J. Am. Chem. Soc. 2001, 123, 10039-10045. d) O. Varnavski, X. Yan, O. Mongin, M. Blanchard-Desce, T. Goodson, J. Phys. Chem. C 2007, 111, 149-162. 
${ }^{37}$ Gaussian 09, Revision D.01, M. J. Frisch, G. W. Trucks, H. B. Schlegel, G. E. Scuseria, M. A. Robb, J. R. Cheeseman, G. Scalmani, V. Barone, B. Mennucci, G. A. Petersson, H. Nakatsuji, M. Caricato, X. Li, H. P. Hratchian, A. F. Izmaylov, J. Bloino, G. Zheng, J. L. Sonnenberg, M. Hada, M. Ehara, K. Toyota, R. Fukuda, J. Hasegawa, M. Ishida, T. Nakajima, Y. Honda, O. Kitao, H. Nakai, T. Vreven, J. A. Montgomery, Jr., J. E. Peralta, F. Ogliaro, M. Bearpark, J. J. Heyd, E. Brothers, K. N. Kudin, V. N. Staroverov, T. Keith, R. Kobayashi, J. Normand, K. Raghavachari, A. Rendell, J. C. Burant, S. S. Iyengar, J. Tomasi, M. Cossi, N. Rega, J. M. Millam, M. Klene, J. E. Knox, J. B. Cross, V. Bakken, C. Adamo, J. Jaramillo, R. Gomperts, R. E. Stratmann, O. Yazyev, A. J. Austin, R. Cammi, C. Pomelli, J. W. Ochterski, R. L. Martin, K. Morokuma, V. G. Zakrzewski, G. A. Voth, P. Salvador, J. J. Dannenberg, S. Dapprich, A. D. Daniels, O. Farkas, J. B. Foresman, J. V. Ortiz, J. Cioslowski, and D. J. Fox, Gaussian, Inc., Wallingford CT, 2013.

38 ArgusLab 4.0, Mark A. Thompson, Planaria Software LLC, Seattle, WA. http://www.arguslab.com.

${ }^{39}$ a) M. G. Vivas, D. L. Silva, J. Malinge, M. Boujtita, R. Zaleśny, W. Bartkowiak, H. Ångren, S. Canuto, L. De Boni, E. Ishow, C. R. Mendonca, Sci. Rep. 2014, 4, 4447; b) D. Cvejn, E. Michail, I. Polyzos, N. Almonasy, O. Pytela, M. Klikar, T. Mikysek, V. Giannetas, M. Fakis, F. Bureš, F. J. Mater. Chem. C, 2015, 3, 7345-7355.

${ }^{40}$ S. P. Mcllroy, E. Cló, L. Nikolajsen, P. K. Frederiksen, C. B. Nielsen, K. V. Mikkelsen, K. V. Gothelf, P. R. Ogilby, J. Org. Chem. 2005, 70, 1134-1146.

${ }^{41}$ D. F. Eaton, Pure Appl. Chem. 1988, 60, 1107-1114.

${ }^{42}$ C. Xu, W. W. Webb, J. Opt. Soc. Am. B 1996, 13, 481-491.

${ }^{43}$ M. A. Albota, C. Xu, W. W. Webb, Appl. Opt. 1998, 37, 7352-7356. 


\begin{tabular}{ccccc}
\hline & $\mathrm{UV} / \mathrm{vis} \lambda_{\max }, \mathrm{nm}$ & $\mathrm{PL}$ & & Stokes shift $^{c}$ \\
$\mathrm{Compd}^{a}$ & $\left(\varepsilon, \mathrm{mM}^{-1} \cdot \mathrm{cm}^{-1}\right)$ & $\lambda_{\max }, \mathrm{nm}$ & $\Phi_{\mathrm{F}}{ }^{b}$ & $\mathrm{~cm}^{-1}$ \\
\hline $\mathbf{1}$ & $332(72.5), 401(86.0)$ & 577 & 0.55 & 7610 \\
$\mathbf{2}$ & $363 \operatorname{sh}(56.7), 422(89.2)$ & 587 & 0.58 & 6660 \\
$\mathbf{3}$ & $345 \mathrm{sh}(69.5), 413(108.2)$ & 630 & 0.37 & 8340 \\
$\mathbf{4}$ & $370 \mathrm{sh}(60.6), 436(108.3)$ & 617 & 0.38 & 6730 \\
$\mathbf{5}$ & $339(53.4), 404(74.5)$ & 551 & 0.67 & 6600
\end{tabular}

${ }^{a}$ All spectra were recorded at room temperature at $c=1.0$ to $2.0 \times 10^{-5} \mathrm{M}$ for absorption and $c=1.0 \times$ to $3.0 \times 10^{-6} \mathrm{M}$ for emission. ${ }^{b}$ Fluorescence quantum yield $( \pm 10 \%)$ determined relative to 9,10 -bisphenylethynyl-anthracene in cyclohexane $\left(\Phi_{\mathrm{F}}=1.00\right) .{ }^{1 \mathrm{c}}$ Calculated using less energetic absorption band.

1 I. B. Berlman, (1971) "Handbook of Fluorescence Spectra of Aromatic Molecules," Academic Press, N.Y. 


\begin{tabular}{|c|c|c|c|c|c|c|c|}
\hline Compd & $\begin{array}{c}\text { Hexane } \\
\mathrm{E}_{\mathrm{T}}(30)^{\mathrm{a}}=31.0 \\
\lambda_{\max }, \mathrm{nm}\end{array}$ & $\begin{array}{c}\text { Toluene } \\
\mathrm{E}_{\mathrm{T}}(30)^{\mathrm{a}}=33.9 \\
\lambda_{\max }, \mathrm{nm}\end{array}$ & $\begin{array}{c}\text { Dioxane } \\
\mathrm{E}_{\mathrm{T}}(30)^{\mathrm{a}}=36.0 \\
\lambda_{\max }, \mathrm{nm}\end{array}$ & $\begin{array}{c}\mathrm{THF} \\
\mathrm{E}_{\mathrm{T}}(30)^{\mathrm{a}}=37.4 \\
\lambda_{\max }, \mathrm{nm}\end{array}$ & $\begin{array}{c}\mathrm{CH}_{2} \mathrm{Cl}_{2} \\
\mathrm{E}_{\mathrm{T}}(30)^{\mathrm{a}}=40.7 \\
\lambda_{\text {max }}, \mathrm{nm}\end{array}$ & $\begin{array}{c}\text { Acetone } \\
\mathrm{E}_{\mathrm{T}}(30)^{\mathrm{a}}=42.2 \\
\lambda_{\max }, \mathrm{nm}\end{array}$ & $\begin{array}{c}\mathrm{MeCN} \\
\mathrm{E}_{\mathrm{T}}(30)^{\mathrm{a}}=45.6 \\
\lambda_{\max }, \mathrm{nm}\end{array}$ \\
\hline 1 & 441 & 463 & 479 & 539 & 577 & $-b$ & $-^{b}$ \\
\hline 2 & 468,493 & 487 & 501 & 551 & 587 & 618 & $-b$ \\
\hline 3 & 458 & 484 & 499 & 576 & 630 & 664 & $-b$ \\
\hline 4 & 485,516 & 508,535 & 533 & 580 & 617 & 635 & $-b$ \\
\hline 5 & 432 & 448 & 461 & 513 & 551 & 581 & 612 \\
\hline
\end{tabular}

${ }^{a}$ Dimroth-Reichardt polarity parameter, $\mathrm{kJ} \cdot \mathrm{mol}^{-1} \cdot{ }^{b}$ no signal detected 


\begin{tabular}{|c|c|c|c|c|}
\hline Compound & $\lambda_{1 P A} / \mathrm{nm}$ & $\lambda_{2 P A} / \mathrm{nm}$ & $\delta_{\max } / \mathrm{GM}^{a}$ & $\Phi \times \delta_{\max } / \mathrm{GM}$ \\
\hline $\mathbf{1}$ & 401 & 760 & 256 & 141 \\
\hline $\mathbf{2}$ & 422 & 800 & 427 & 248 \\
\hline $\mathbf{3}$ & 413 & 780 & 239 & 88 \\
\hline $\mathbf{4}$ & 436 & 760 & 416 & 158 \\
\hline $\mathbf{5}$ & 404 & 740 & 158 & 106 \\
\hline
\end{tabular}




\begin{tabular}{|c|c|c|c|c|c|c|c|c|c|c|}
\hline Comp. & $\begin{array}{c}E_{1 / 2}^{(\mathrm{ox} 1)} \\
/ \mathrm{V}^{a}\end{array}$ & $\begin{array}{c}E_{1 / 2}(\operatorname{red} 1) \\
/ \mathrm{V}^{a}\end{array}$ & $\begin{array}{c}E_{\text {НОМо }} \\
/ \mathrm{eV}^{b}\end{array}$ & $\begin{array}{c}E_{\text {LUMO }} \\
/ \mathrm{eV}^{b}\end{array}$ & $\begin{array}{c}E \mathrm{~g}^{\text {elchem }} \\
/ \mathrm{eV}^{c}\end{array}$ & $\begin{array}{c}E \mathrm{~g}^{\text {optical }} \\
/ \mathrm{eV}\end{array}$ & $\begin{array}{c}E_{\mathrm{HOMO}}{ }^{\mathrm{DFT}} \\
/ \mathrm{eV}^{f}\end{array}$ & $\begin{array}{c}E_{\mathrm{LUMO}}{ }^{\mathrm{DFT}} \\
/ \mathrm{eV}^{f}\end{array}$ & $\begin{array}{l}E \mathrm{~g}^{\mathrm{DFT}} \\
/ \mathrm{eV}^{f}\end{array}$ & $\begin{array}{l}\mu_{\mathrm{G}} \\
/ \mathrm{D}^{f}\end{array}$ \\
\hline 1 & 0.61 & -1.91 & -5.41 & -2.89 & 2.52 & 2.63 & -5.42 & -2.72 & 2.70 & 3.62 \\
\hline 2 & $-{ }^{d}$ & -1.70 & - & -3.10 & - & 2.53 & -5.32 & -2.74 & 2.58 & 0.08 \\
\hline 3 & 0.63 & -1.77 & -5.43 & -3.03 & 2.40 & 2.57 & -5.31 & -2.77 & 2.54 & 4.79 \\
\hline 4 & $0.69^{e}$ & -1.72 & -5.49 & -3.08 & 2.41 & 2.43 & -5.23 & -2.92 & 2.31 & 1.40 \\
\hline 5 & 0.64 & -2.02 & -5.44 & -2.78 & 2.66 & 2.71 & -5.33 & -2.44 & 2.89 & 0.48 \\
\hline
\end{tabular}

$E_{\mathrm{HOMO}}-E_{\mathrm{LUMO}} ;{ }^{d}$ Undistinguishable wave; ${ }^{e}$ Irreversible peak, stand for $E \mathrm{p} ;{ }^{f}$ All data were calculated by DFT method HCTH/6-311+G(d)//B3LYP/6-311++G(2d,p).

${ }^{1}$ A. A. Isse, A. Gennaro, J. Phys. Chem. B, 2010, 114, 7894-7899. 


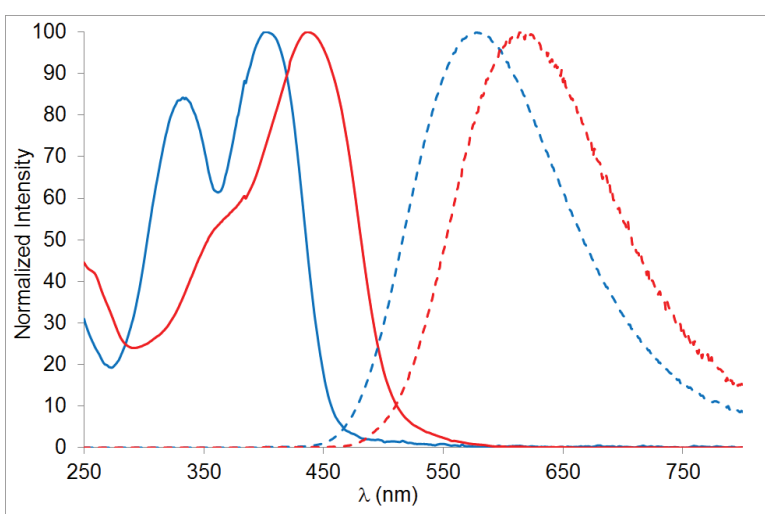


<smiles>O=Cc1ccc(Br)cc1/C=C/c1ccc(Br)cc1</smiles>

$6(78 \%)$<smiles>Brc1ccc(/C=C/c2cnc3ccccc3n2)cc1</smiles>
$8(55 \%)$

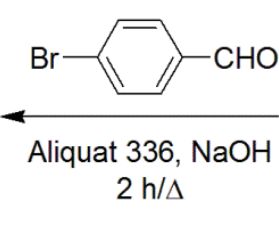

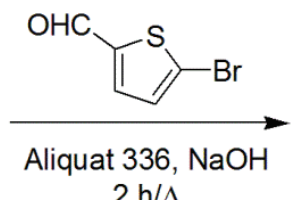

$2 \mathrm{~h} / \Delta$<smiles>Cc1ccncn1</smiles><smiles>Cc1cnc2ccccc2n1</smiles>
$2 \mathrm{~h} / \Delta$<smiles>Brc1ccc(/C=C/c2ccncn2)s1</smiles>

$7(79 \%)$<smiles>Brc1ccc(/C=C/c2cnc3ccccc3n2)s1</smiles>

9 (54 \%) 

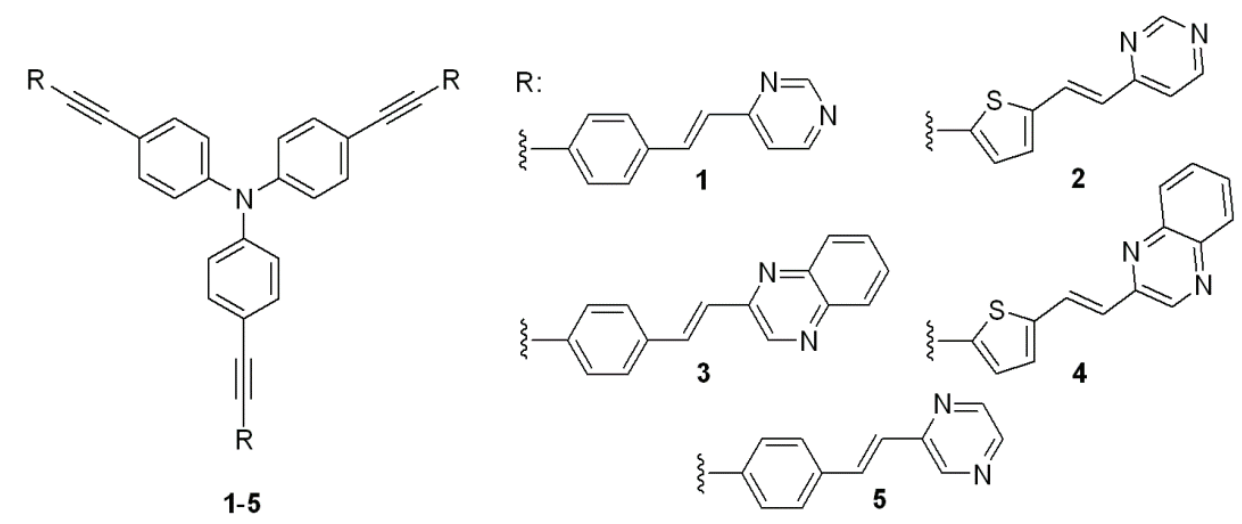


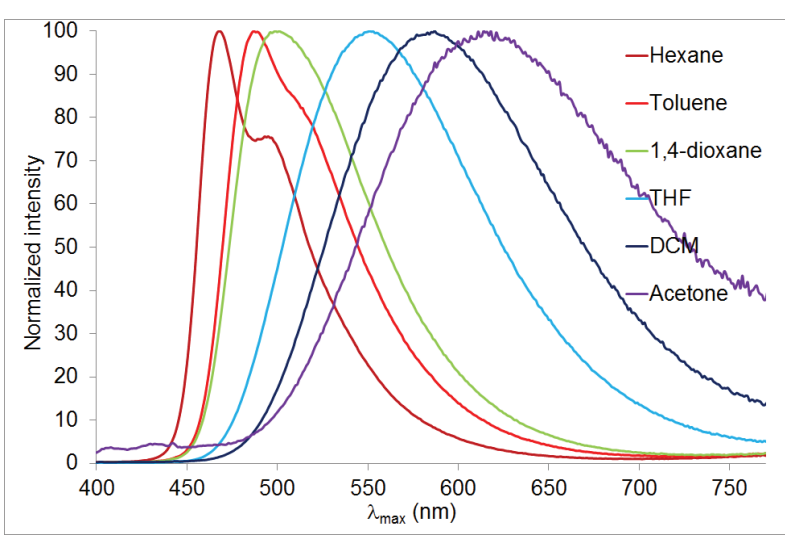




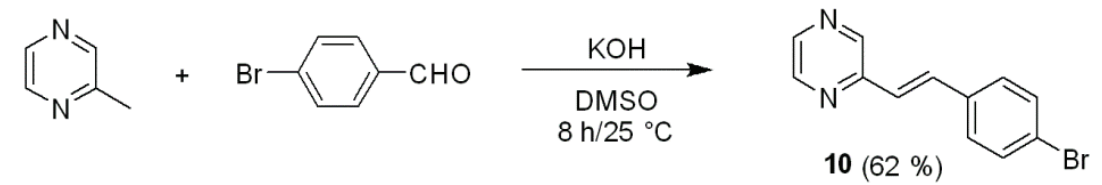




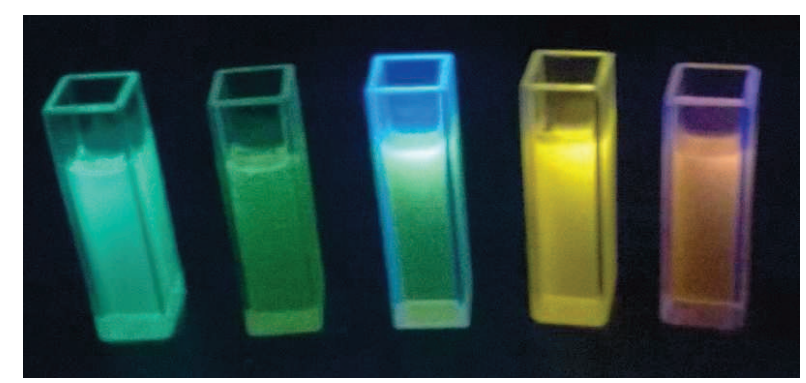



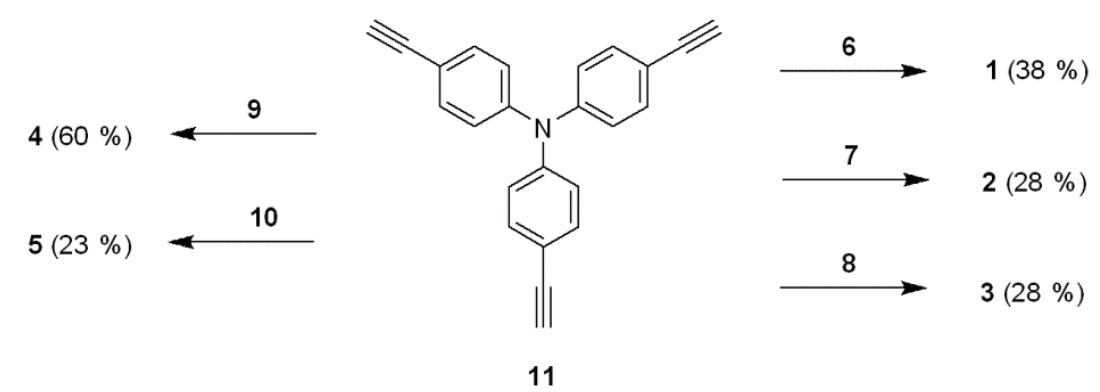


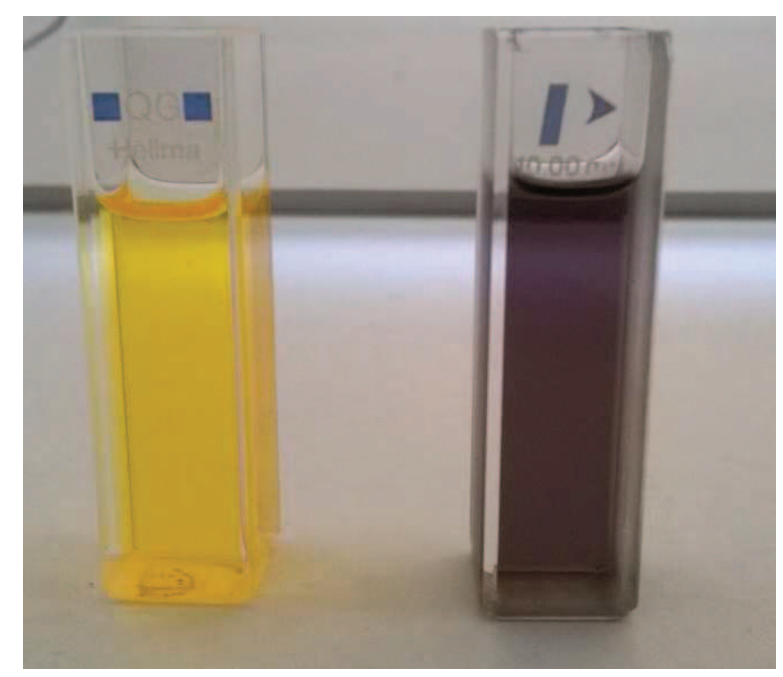




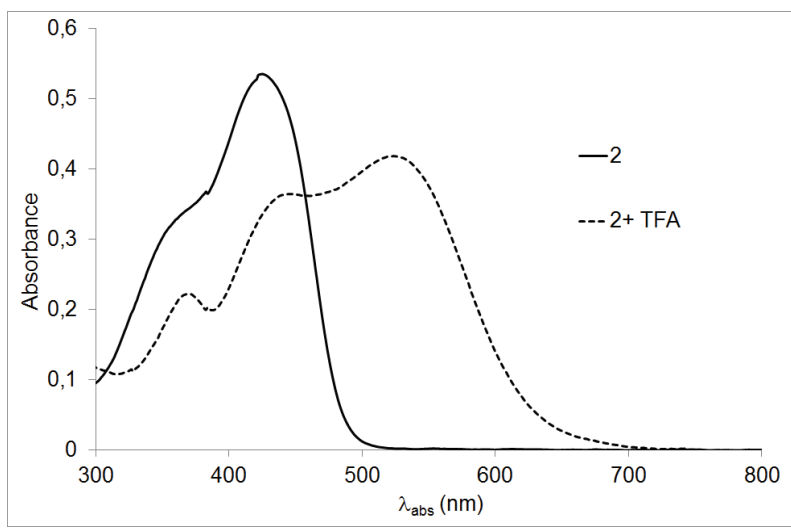




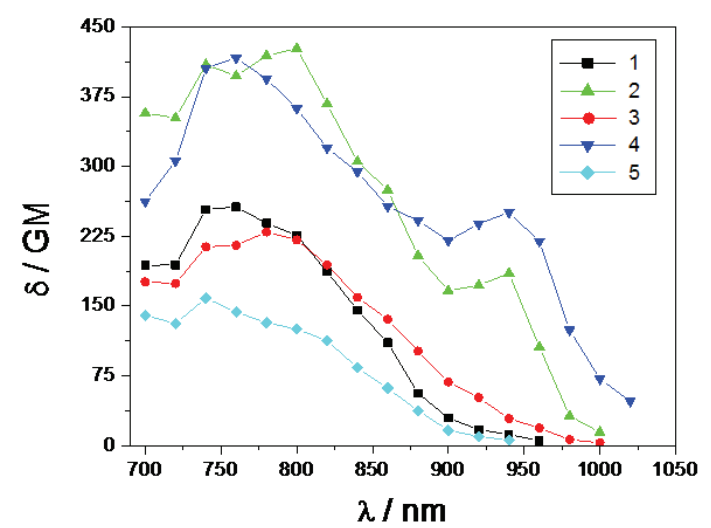




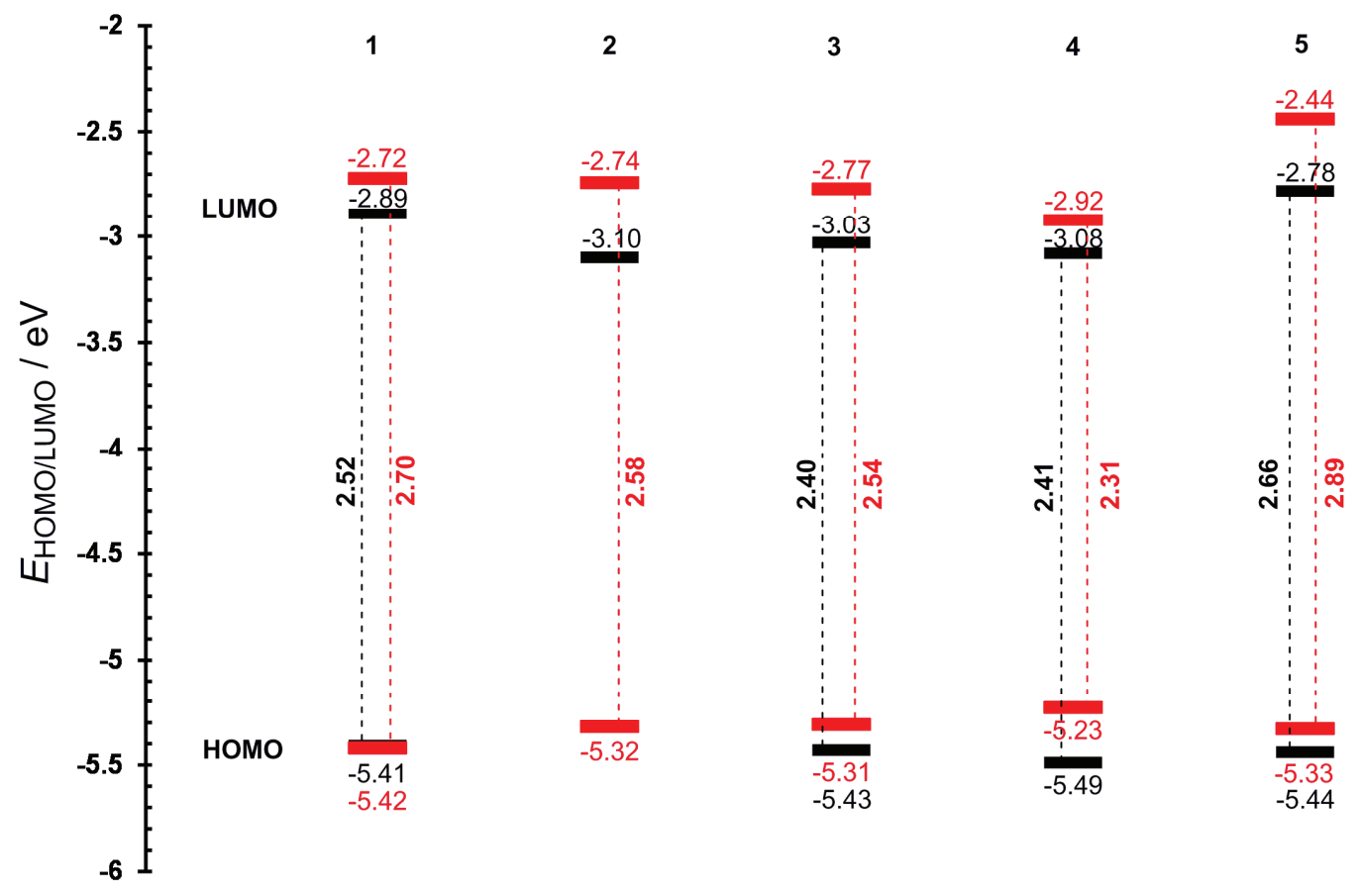



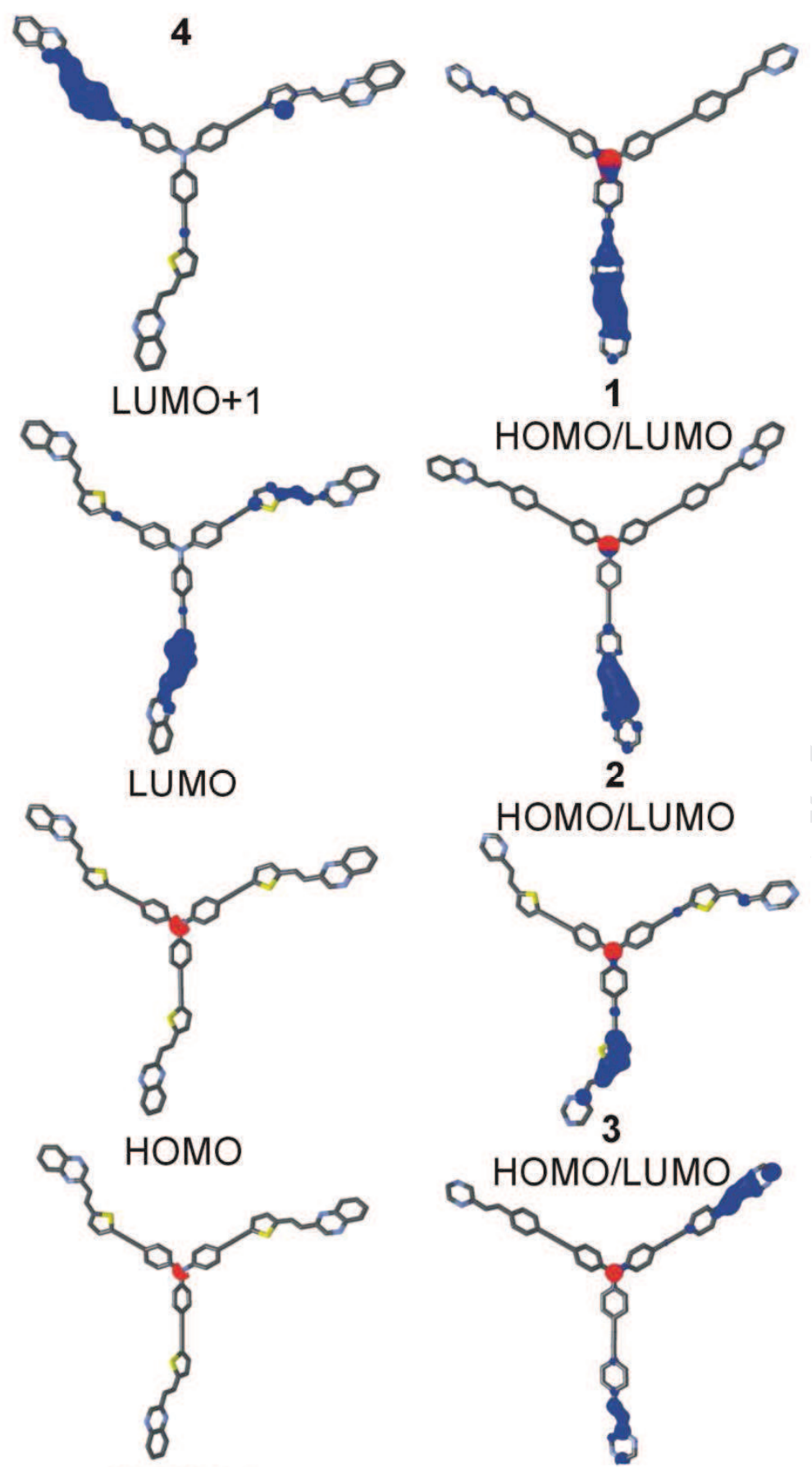

HOMO-1

5

HOMO/LUMO 
Octupolar molecules with central triphenylamine and peripheral diazine were prepared.

A strong positive emission solvatochromism is observed.

These materials exhibit two-photon absorption properties. 


\section{SUPPORTING INFORMATION}

\section{Star-shaped molecules with triphenylamine core, diazine peripheral groups and extended $\pi$-conjugated linkers}

Daniel Cvejn, ${ }^{[\mathrm{a}, \mathrm{b}]}$ Sylvain Achelle, ${ }^{[\mathrm{a}]}$ Oldřich Pytela,${ }^{[\mathrm{b}]}$ Jean-Pierre Malval, ${ }^{[\mathrm{c}]}$ Arnaud Spangenberg, ${ }^{[\mathrm{c}]}$ Nolwenn Cabon, ${ }^{[\mathrm{a}]}$ Filip Bureš, ${ }^{*[\mathrm{~b}]}$ and Françoise Robin-le Guen ${ }^{[\mathrm{a}]}$

${ }^{[a]}$ Institut des Sciences Chimiques de Rennes UMR CNRS 6226, IUT de Lannion, rue Edouard Branly, BP 30219, F22302 Lannion Cedex, France. ${ }^{[\mathrm{b}]}$ Institute of Organic Chemistry and Technology, Faculty of Chemical Technology, University of Pardubice, Studentská 573, Pardubice, 53210, Czech Republic. ${ }^{[c]}$ Institut de Science des Matériaux de Mulhouse UMR CNRS 7361, Université de Haute-Alsace 15 rue Jean Starcky, 68057 Mulhouse, France

Corresponding authors: *E-mails: sylvain.achelle@univ-rennes1.fr; filip.bures@upce.cz

\section{Table of contents}

1. NMR spectra for compounds 1-5 (p S2-S6).

2. NMR spectra for compounds $\mathbf{9}$ and $\mathbf{1 0}$ (p S7-S8).

3. UV/Vis and photoluminescence (PL) data of compounds 1-5 in Toluene (p S9).

4. Emission wavelength as a function of $\mathrm{E}_{\mathrm{T}}(30)$ for compounds 1- 5 (p S10).

5. Fluorescence color changes for $\mathbf{5}$ in various solvents (p S11).

6. Representative CV diagram of compound 5. (p S12)

7. 1PA and 2PA spectra of compounds $\mathbf{1 - 5}(\mathrm{p} \mathrm{S13})$

8. DFT calculations (p S16) 


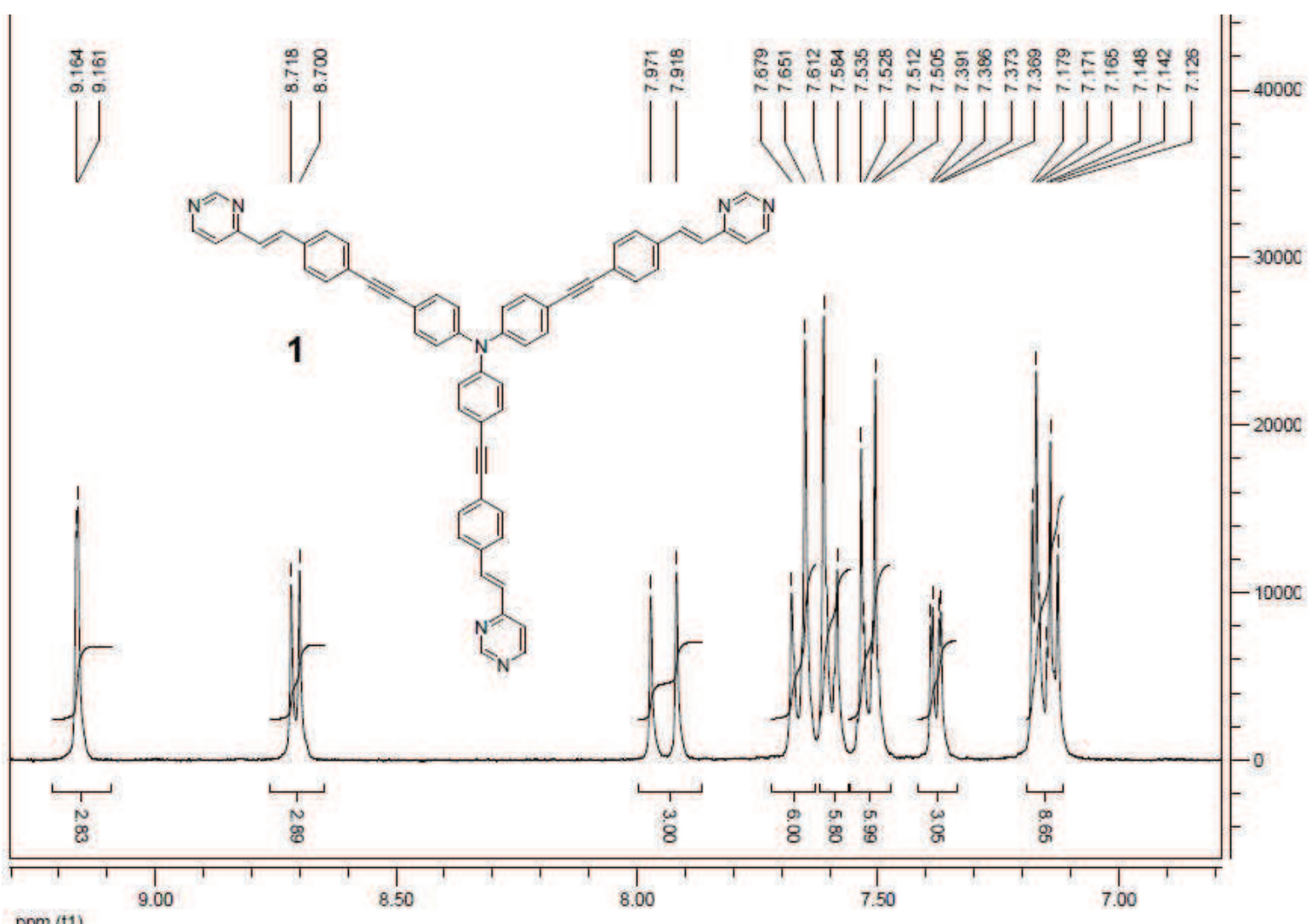

$\mathrm{ppm}(\mathrm{t})$

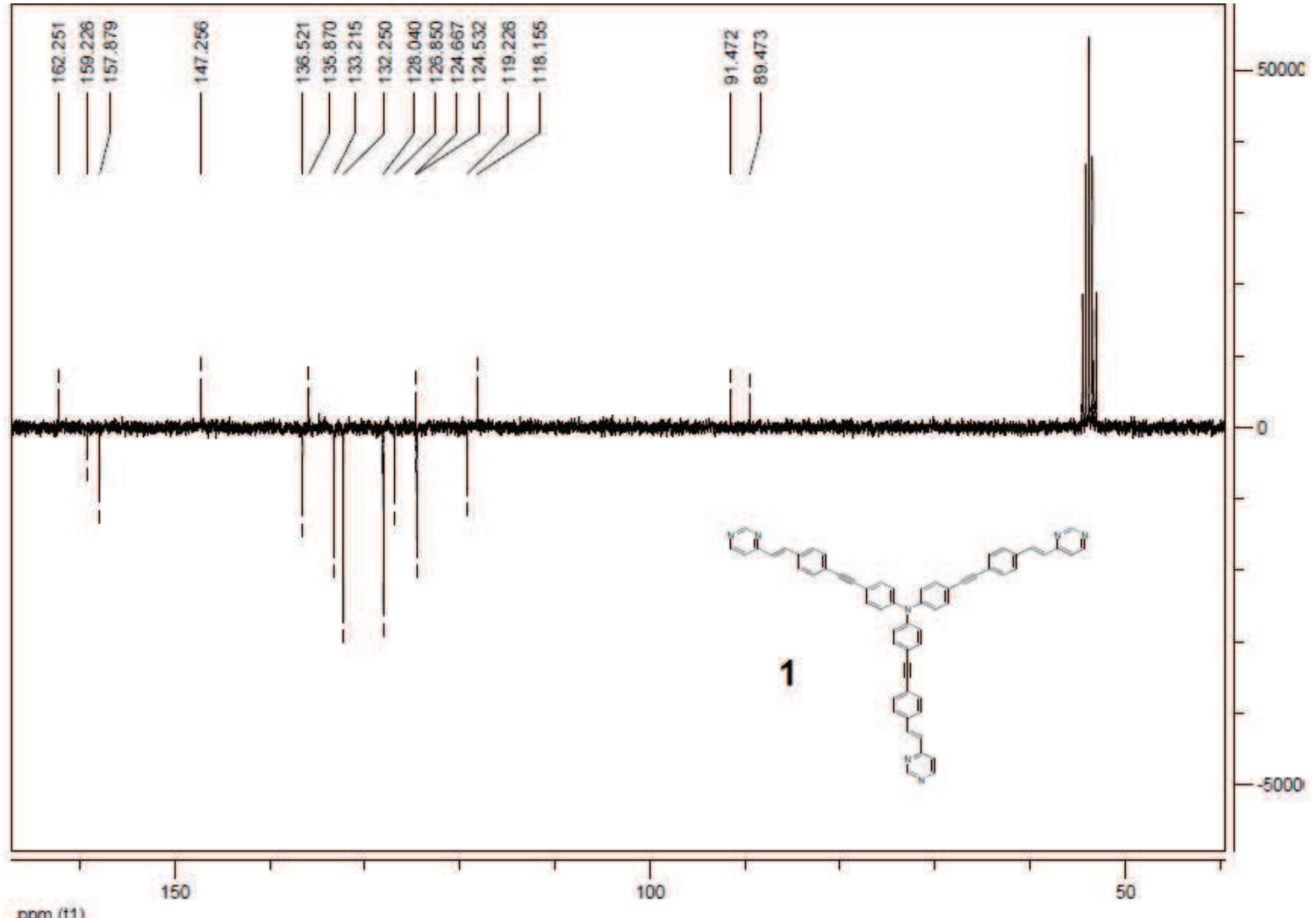

ppm (t1)

${ }^{1} \mathrm{H}$ and ${ }^{13} \mathrm{C}$-NMR spectra of chromophore $1\left(300 / 75 \mathrm{MHz}, \mathrm{CDCl}_{3}\right)$. 

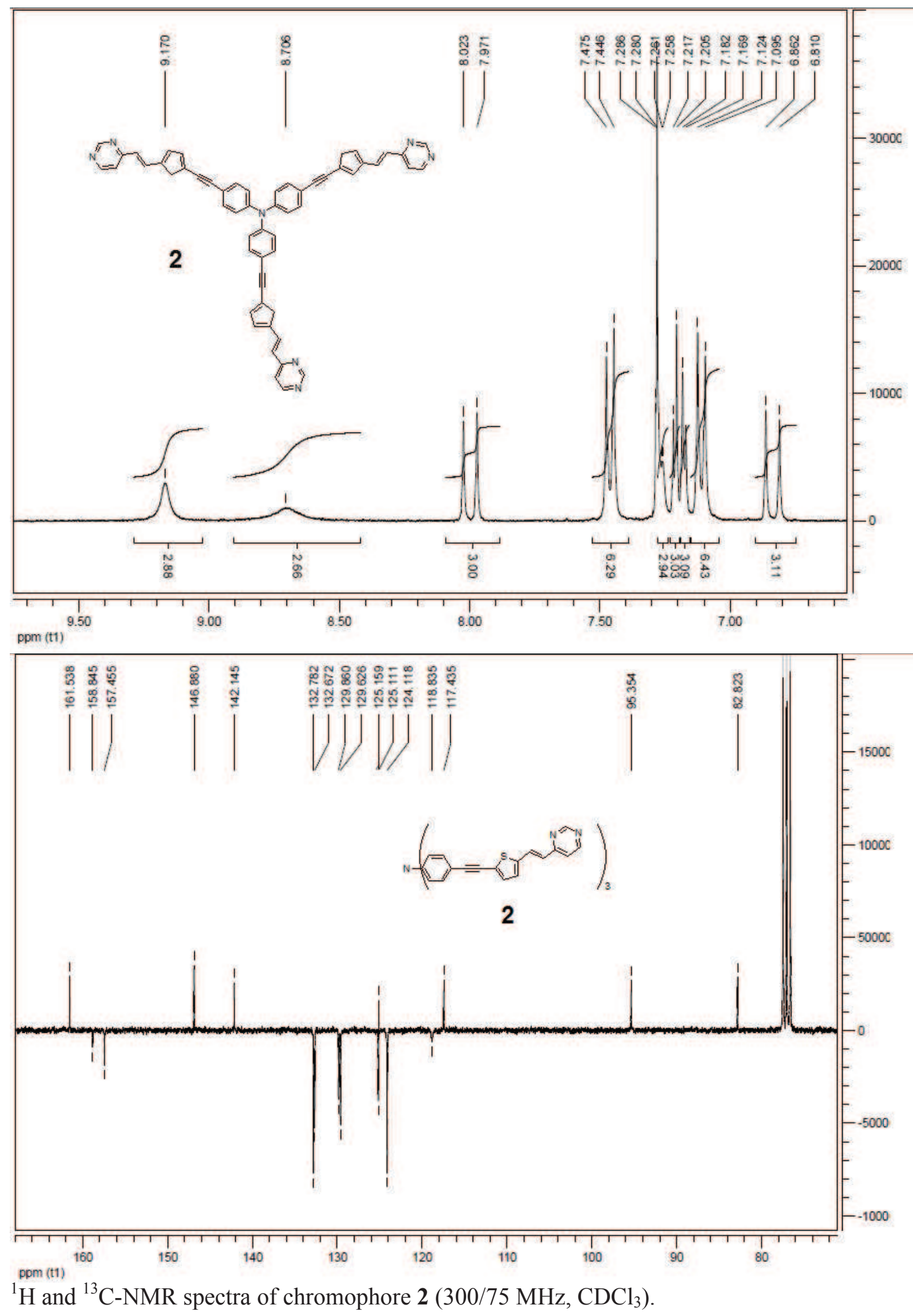

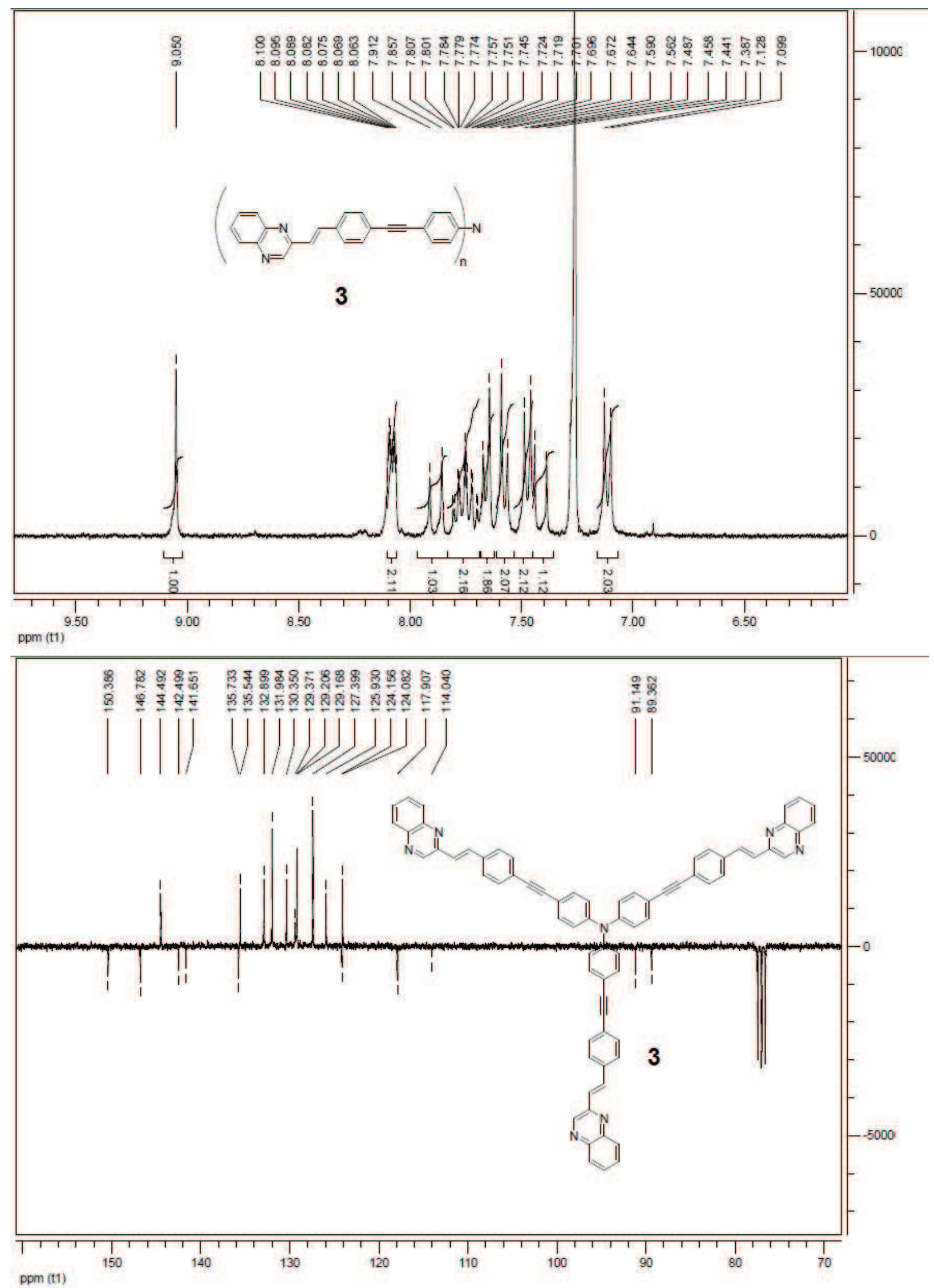

${ }^{1} \mathrm{H}$ and ${ }^{13} \mathrm{C}$-NMR spectra of chromophore 3 (300/75 MHz, $\left.\mathrm{CDCl}_{3}\right)$. 


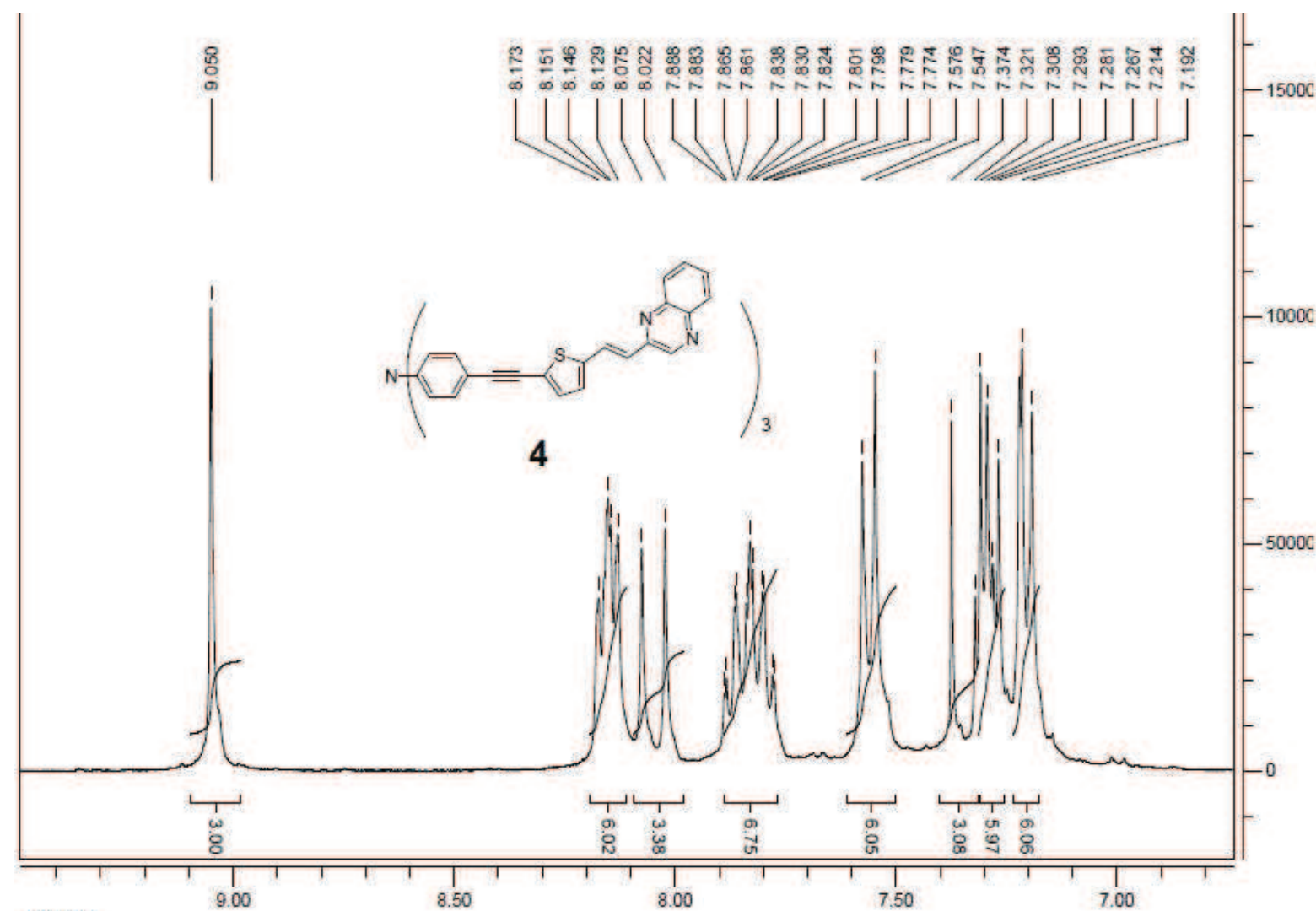

$\mathrm{ppm}(\mathrm{t} 1)$

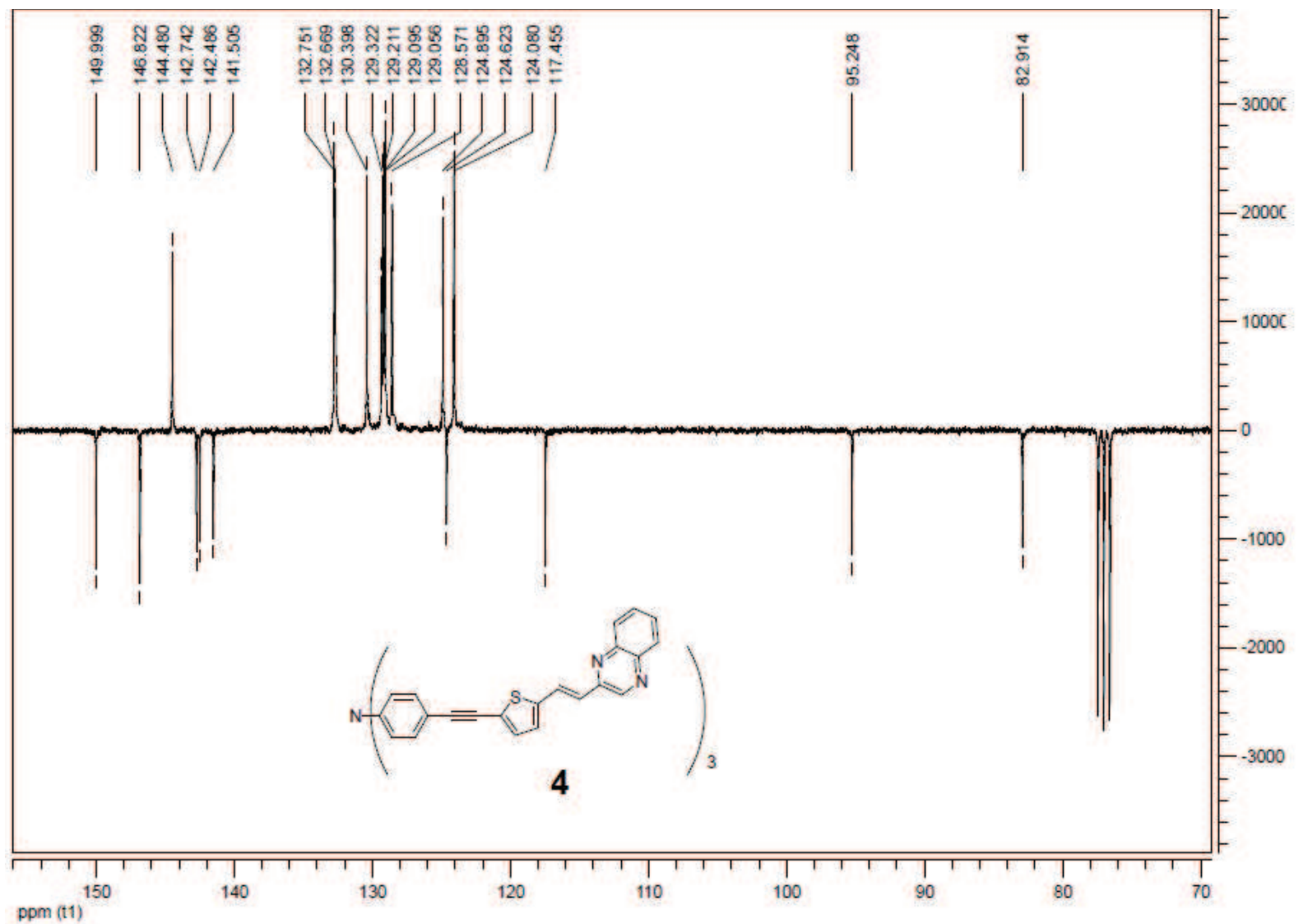

${ }^{1} \mathrm{H}$ and ${ }^{13} \mathrm{C}$-NMR spectra of chromophore $4\left(300 / 75 \mathrm{MHz}, \mathrm{CDCl}_{3}\right)$. 


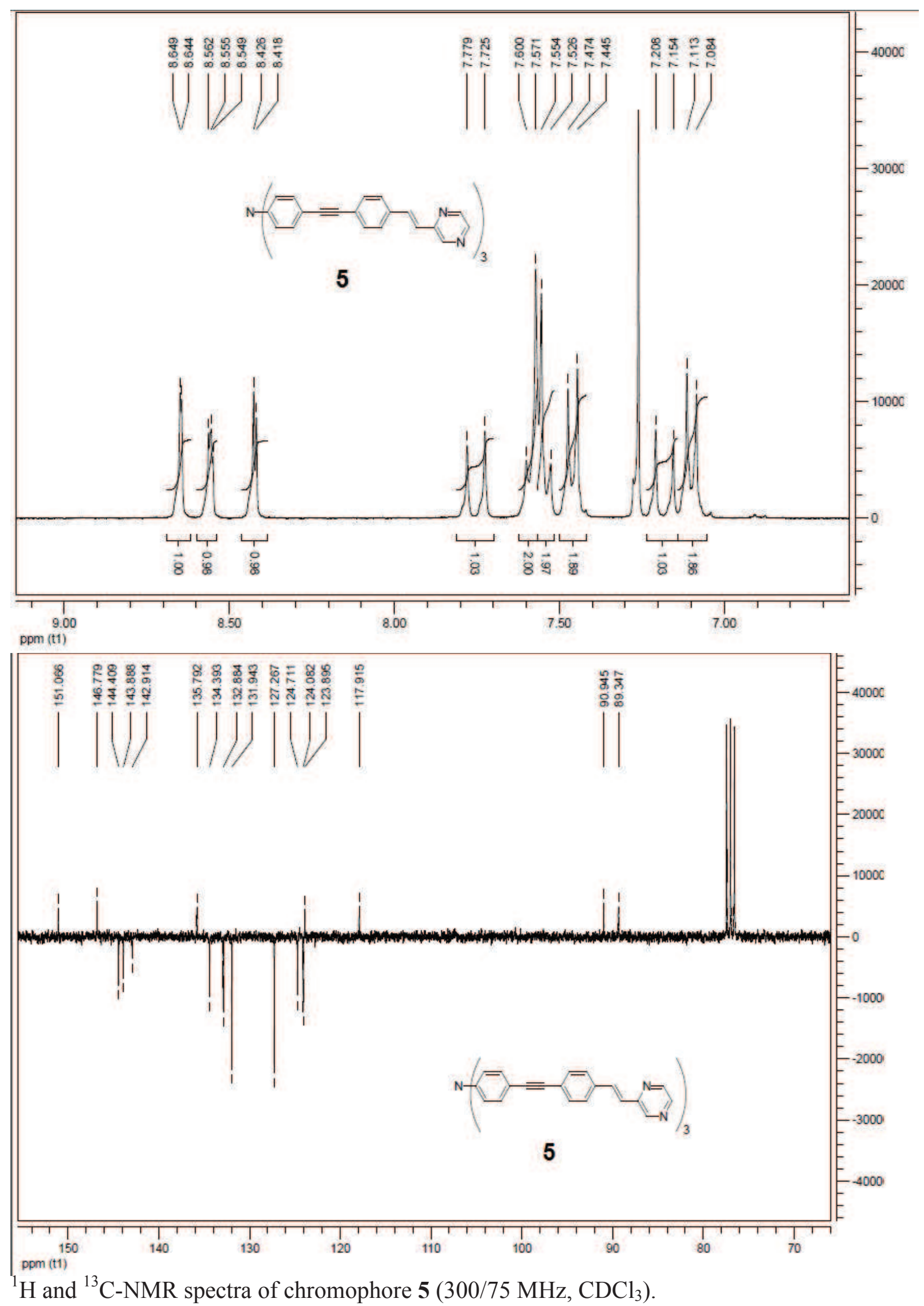




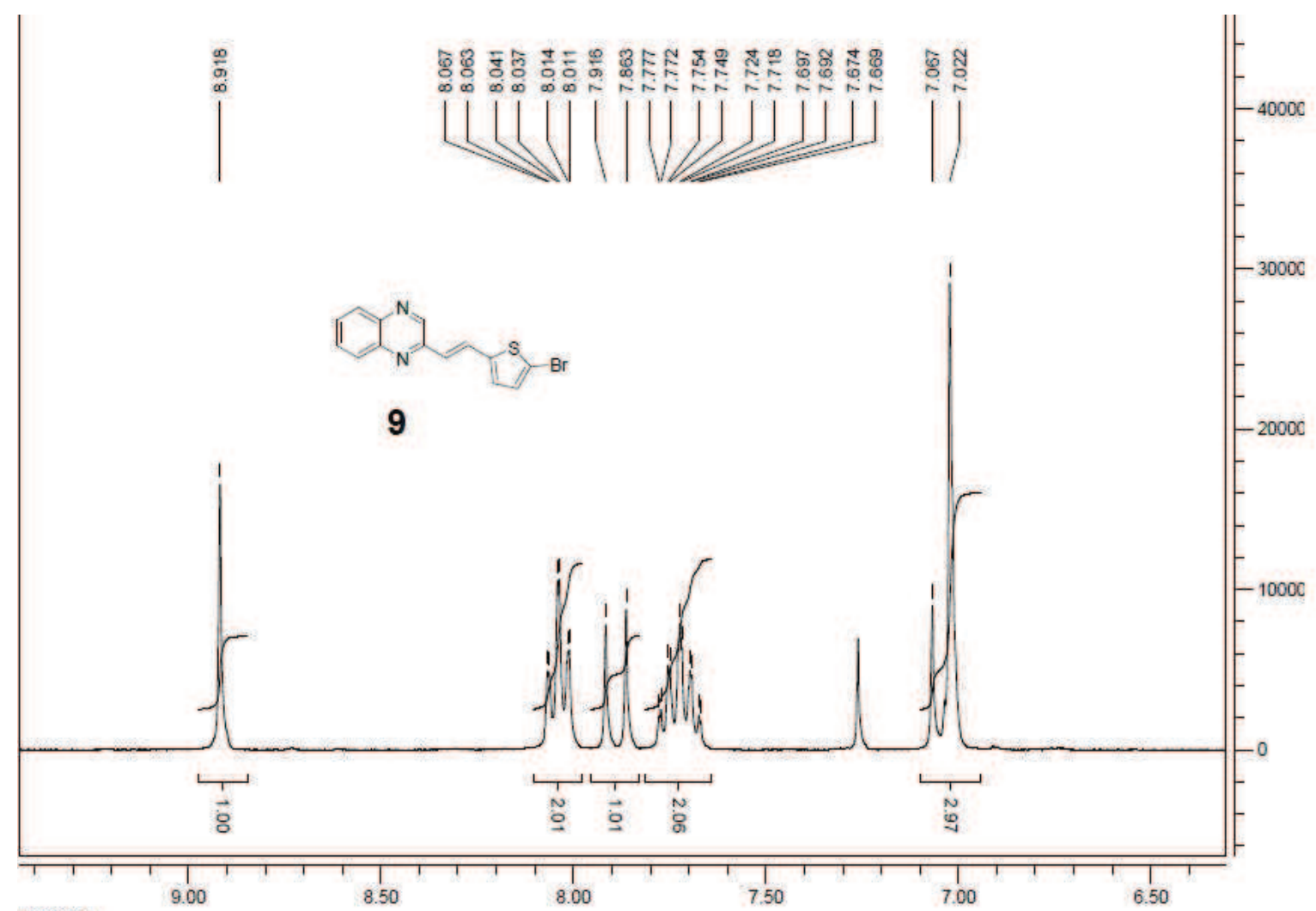

ppm (t1)

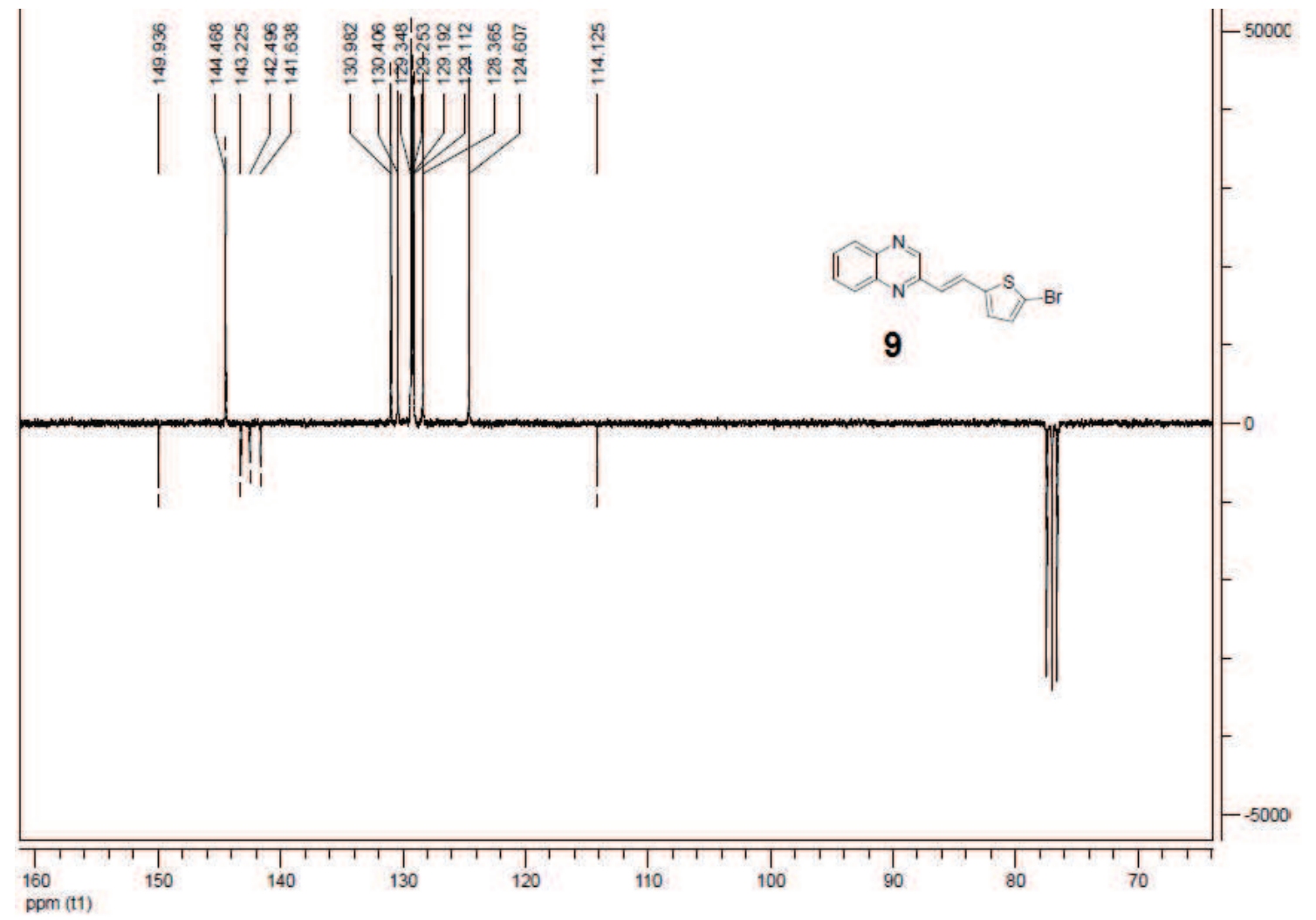

${ }^{1} \mathrm{H}$ and ${ }^{13} \mathrm{C}$-NMR spectra of intermediate $9\left(300 / 75 \mathrm{MHz}, \mathrm{CDCl}_{3}\right)$. 


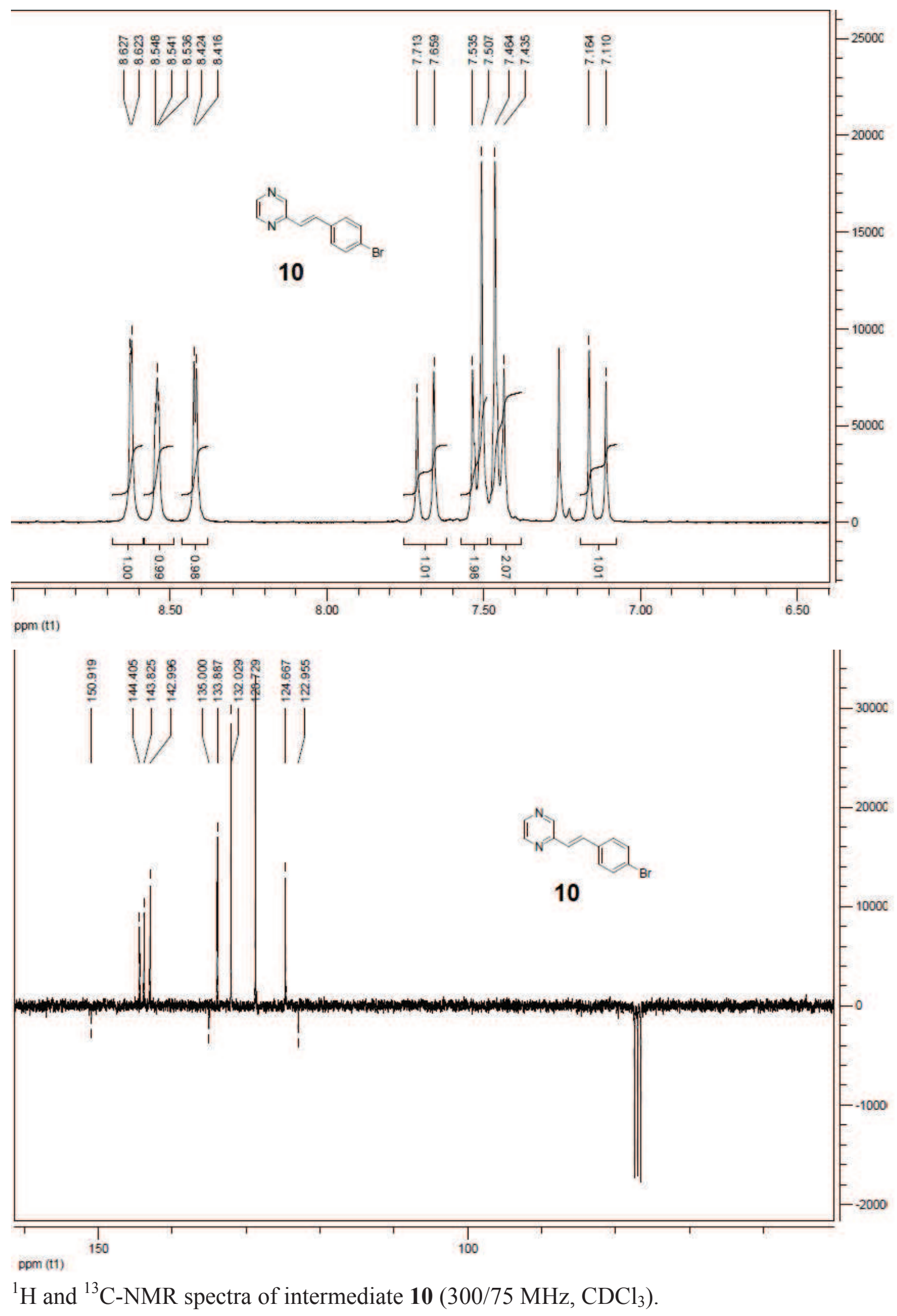




\section{$\mathrm{UV} / \mathrm{V}$ is and photoluminescence (PL) data in Toluene}

\begin{tabular}{ccccc}
\hline & $\mathrm{UV} / \mathrm{vis} \lambda_{\max }, \mathrm{nm}$ & $\mathrm{PL}$ & & Stokes shift $^{c}$ \\
$\mathrm{Compd}^{a}$ & $\left(\varepsilon, \mathrm{mM}^{-1} \cdot \mathrm{cm}^{-1}\right)$ & $\lambda_{\max }, \mathrm{nm}$ & $\Phi_{\mathrm{F}}{ }^{b}$ & $\mathrm{~cm}^{-1}$ \\
\hline $\mathbf{1}$ & $326(74.2), 401(96.8)$ & 463 & 1.00 & 3339 \\
$\mathbf{2}$ & $363(58.3), 422(95.1)$ & 487 & 0.45 & 3163 \\
$\mathbf{3}$ & $344(71.6), 411(120.9)$ & 484 & 0.73 & 3670 \\
$\mathbf{4}$ & $439(103.3)$ & $508,535(\mathrm{sh})$ & 0.51 & 3094 \\
$\mathbf{5}$ & $341(45.4), 397(65.4)$ & 448 & 0.67 & 2867 \\
\hline
\end{tabular}

${ }^{a}$ All spectra were recorded at room temperature at $c=1.0 \times 10^{-5} \mathrm{M}$ to $2.0 \times 10^{-5} \mathrm{M}$ for absorption and $c=1.0 \times 10^{-6} \mathrm{M}$ to $3.0 \times 10^{-6} \mathrm{M}$ for emission. ${ }^{b}$ Fluorescence quantum yield $( \pm 10 \%)$ determined relative to 9,10 -Bis-phenylethynyl-anthracene in cyclohexane $\left(\Phi_{\mathrm{F}}=1.00\right) .{ }^{\mathrm{i}}{ }^{\mathrm{c}}$ calculated using less energetic absorption band. 


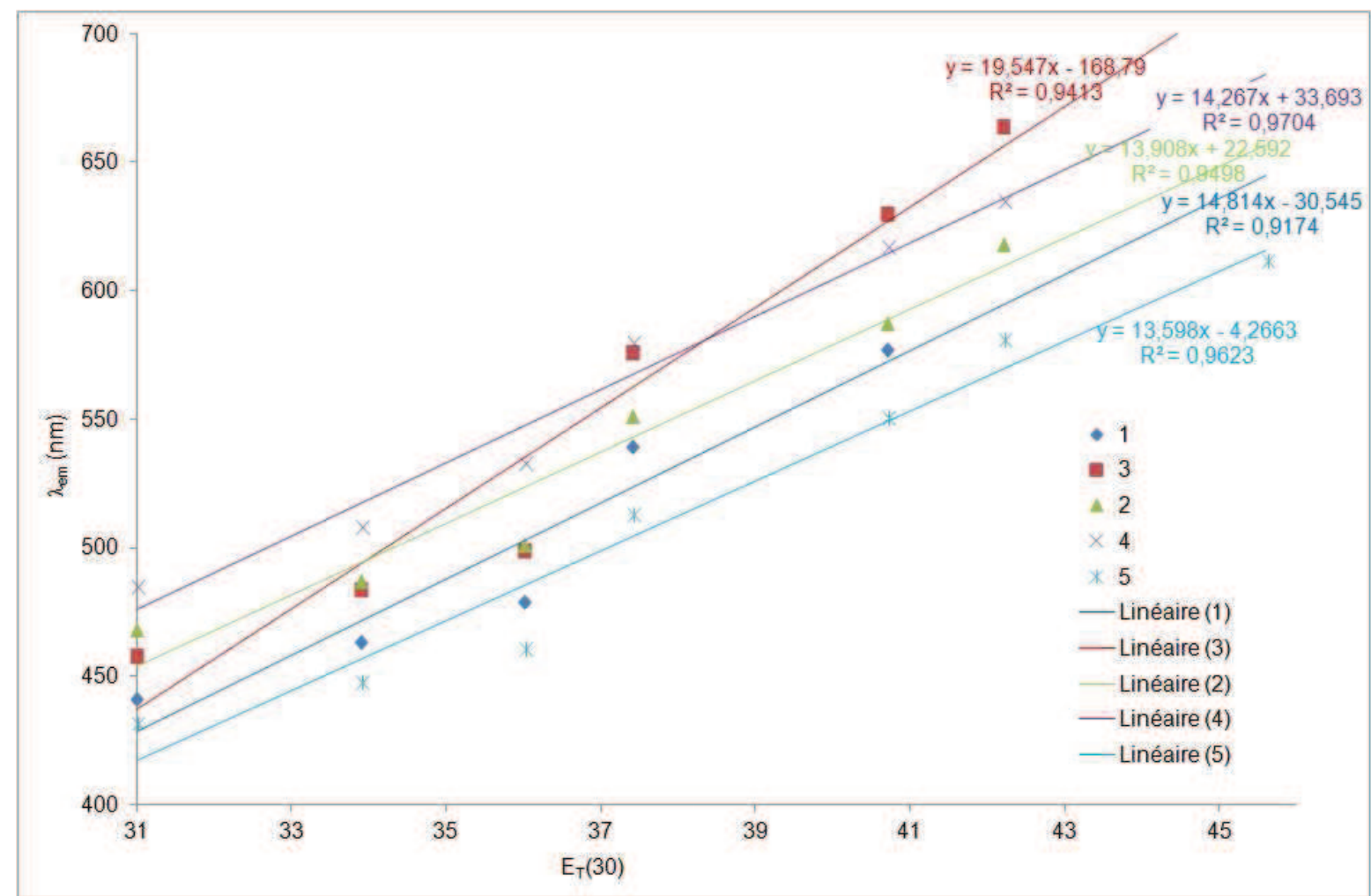

Correlation of the longest-wavelength absorption maxima and $\mathrm{E}_{\mathrm{T}}(30)$ parameters. 


\section{ACCEPTED MANUSCRIPT}

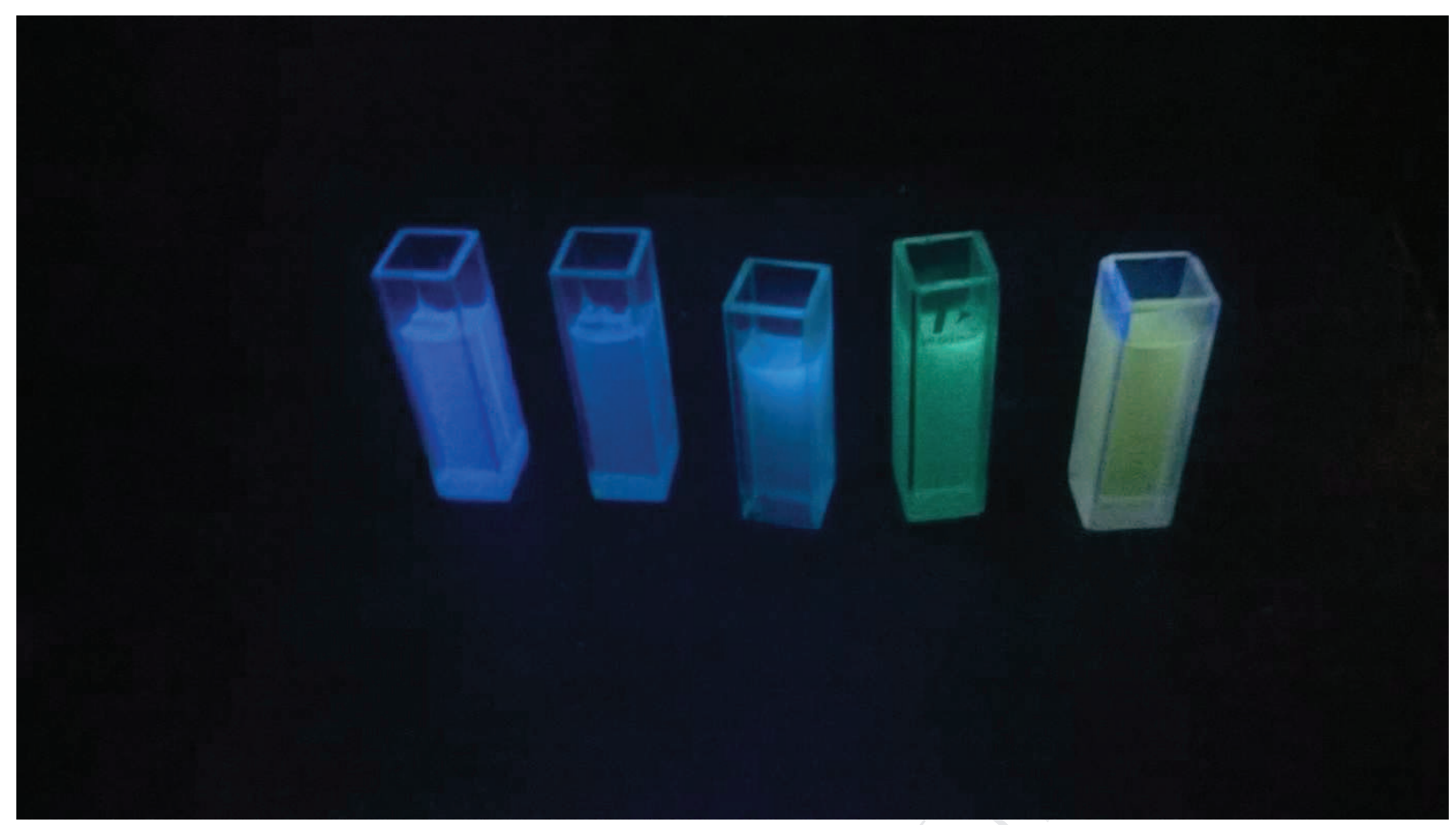

Color of solution of chromophore 5 in various solvents $\left(\mathrm{c}=10^{-5} \mathrm{M}\right)$ taken under irradiation with a handheld UV lamp (365 nm). From left to right: Hexane, Toluene, dioxane, THF, DCM. 


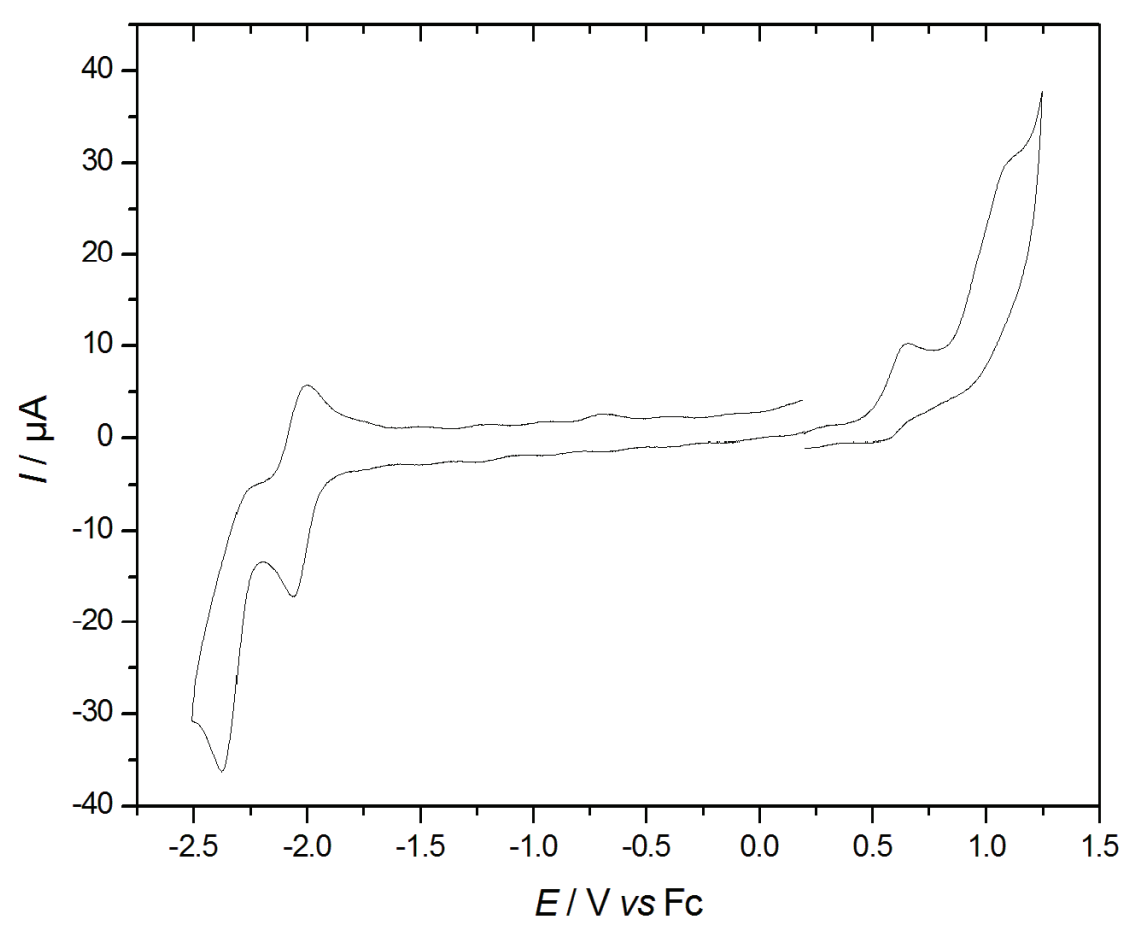

Representative CV diagram of compound 5. 


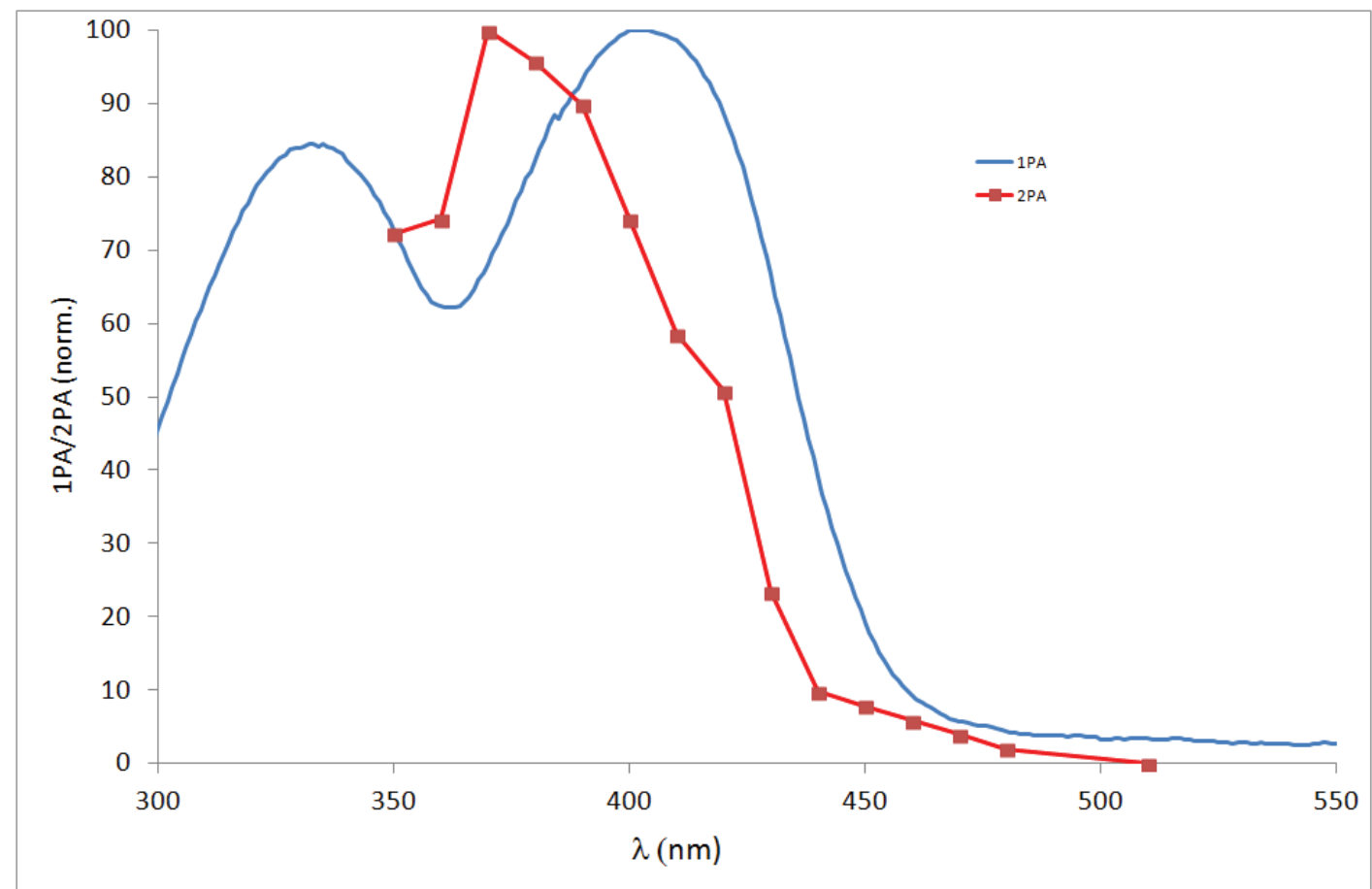

1PA (blue) and 2PA (red) spectra (plotted against half of the excitation wavelength) of 1 in dichloromethane.

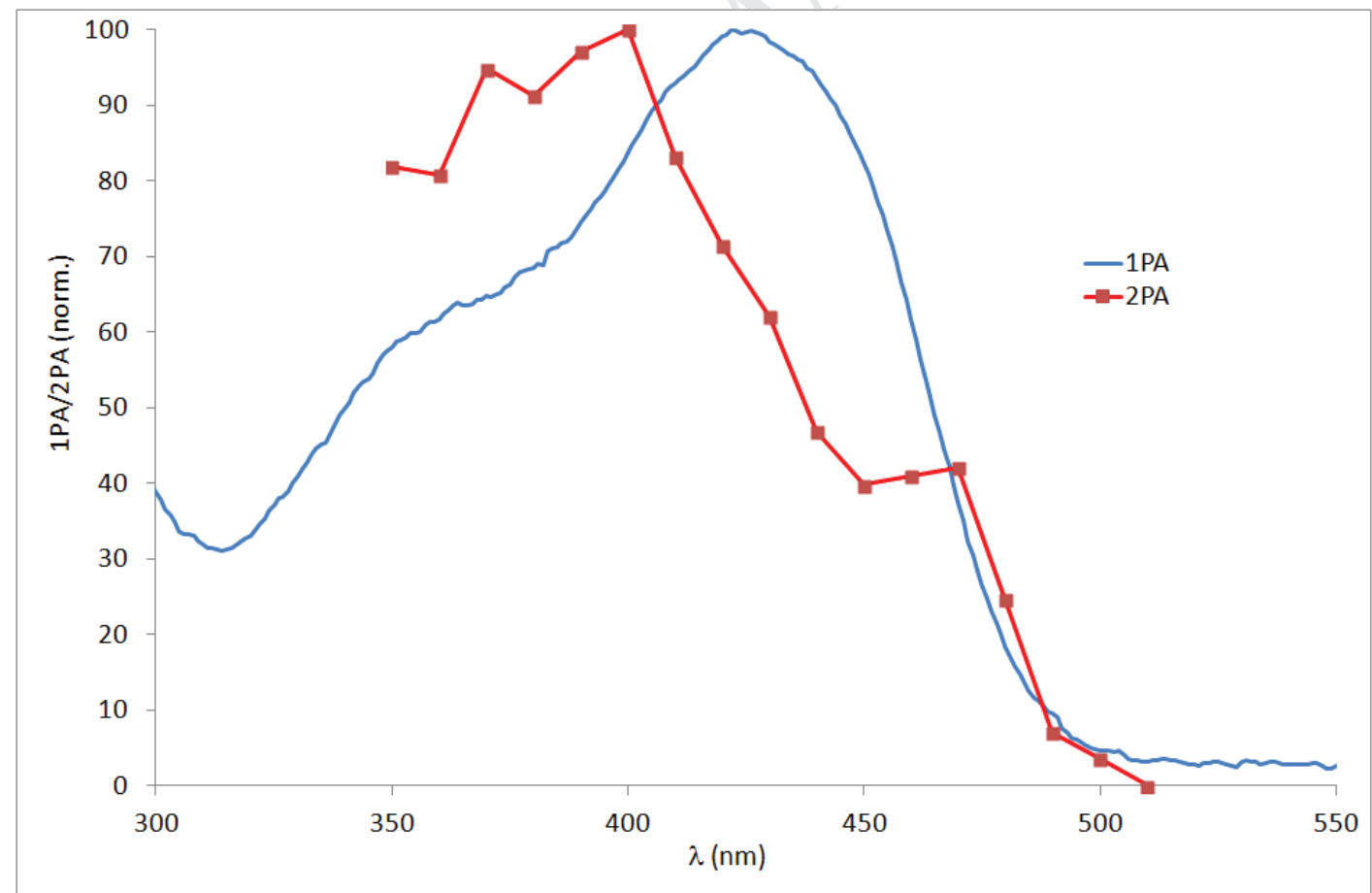

1PA (blue) and 2PA (red) spectra (plotted against half of the excitation wavelength) of 2 in dichloromethane. 


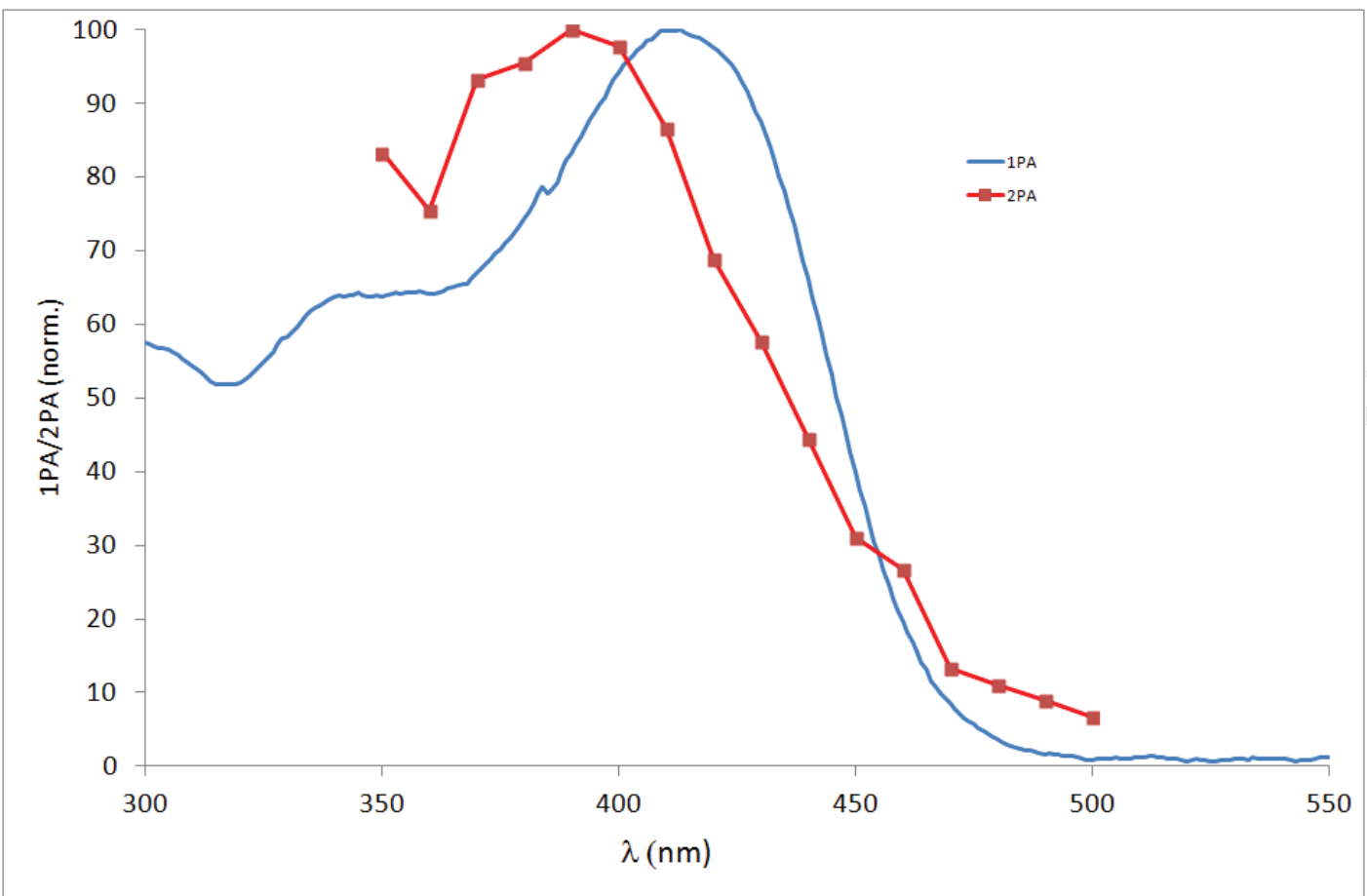

1PA (blue) and 2PA (red) spectra (plotted against half of the excitation wavelength) of $\mathbf{3}$ in dichloromethane.

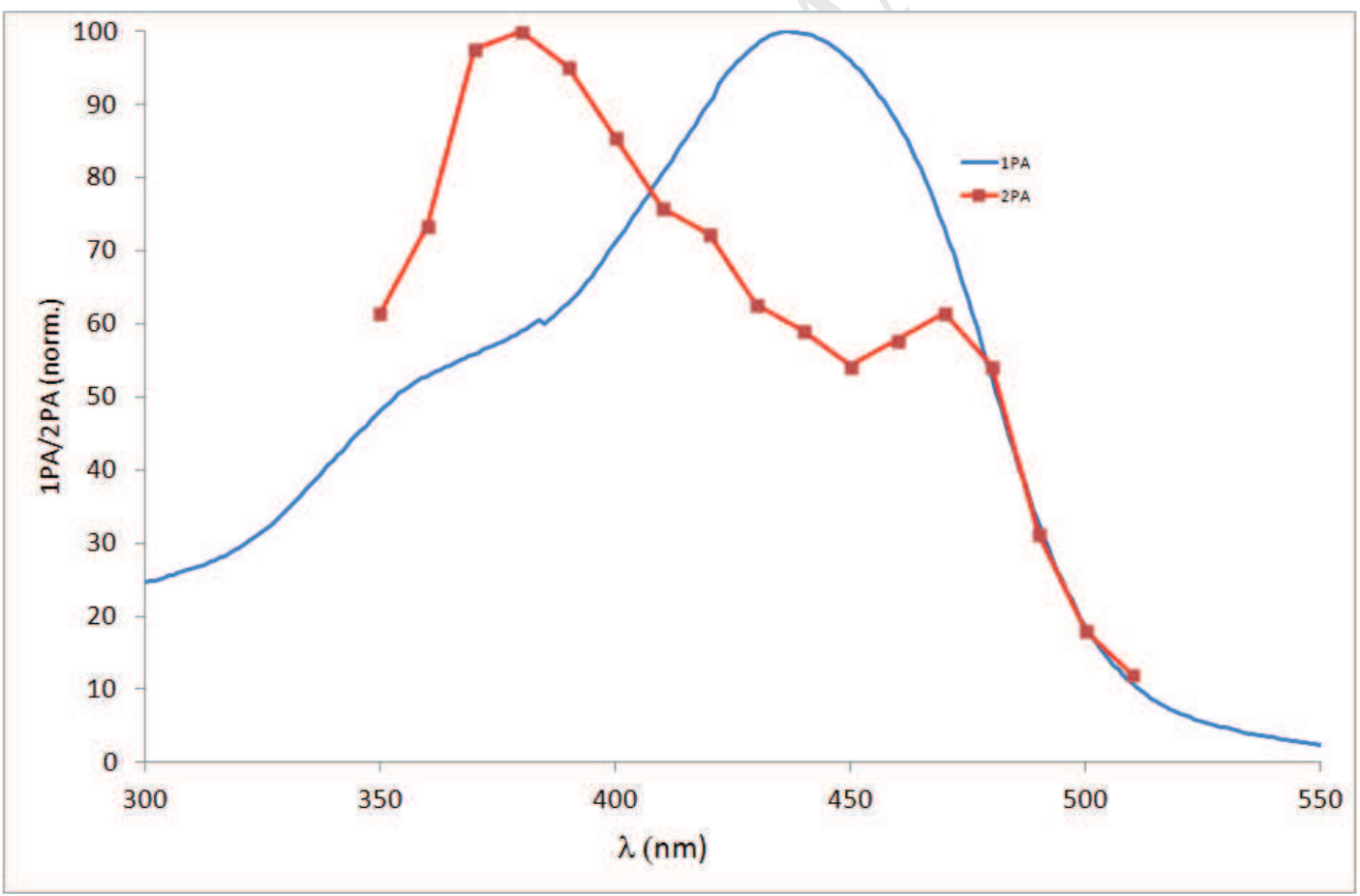

1PA (blue) and 2PA (red) spectra (plotted against half of the excitation wavelength) of 4 in dichloromethane. 


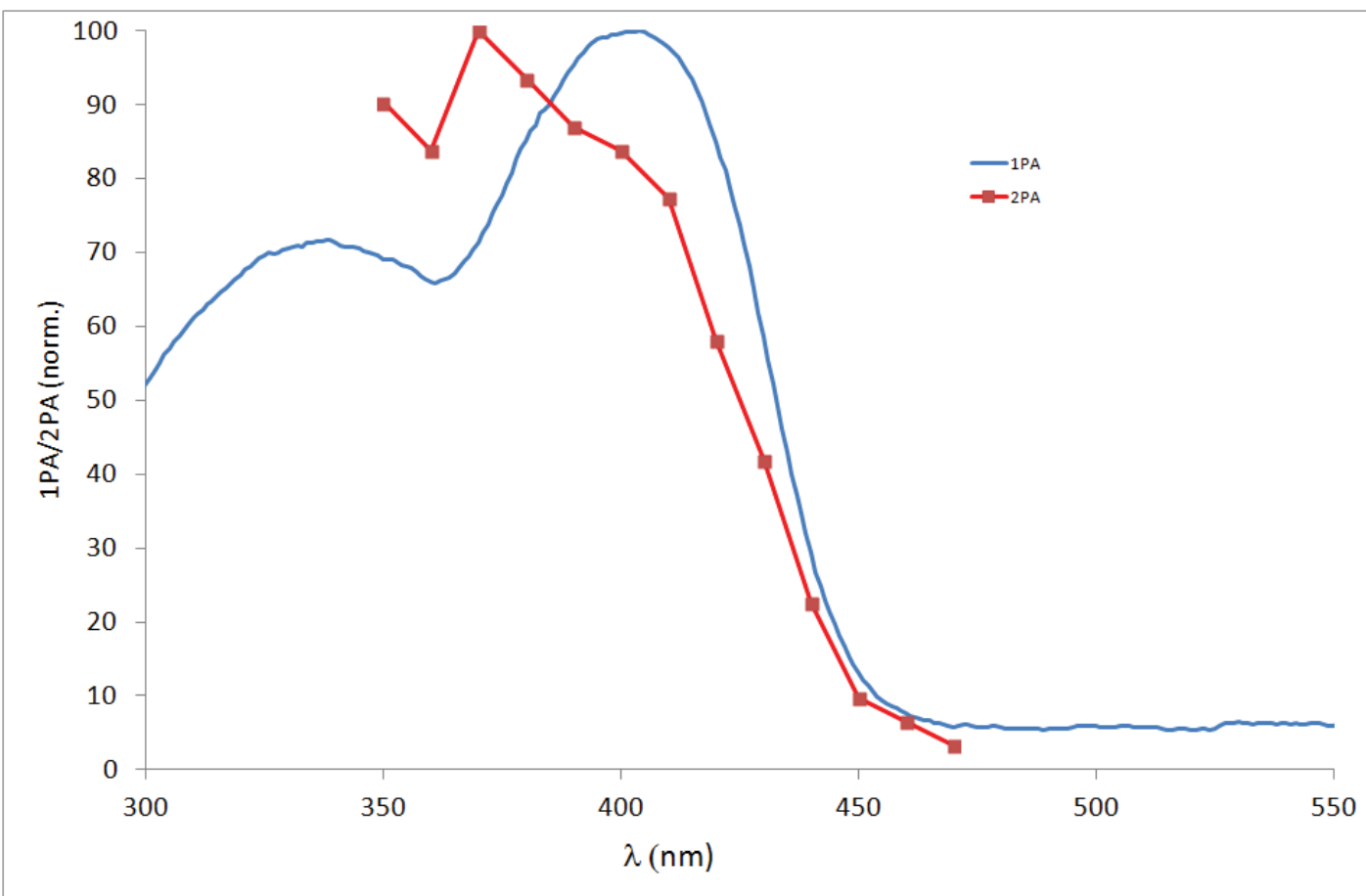

1PA (blue) and 2PA (red) spectra (plotted against half of the excitation wavelength) of 5 in dichloromethane. 


\section{DFT calculations}

All data were calculated by DFT method HCTH/6-311+G(d)//B3LYP/6-311++G(2d,p)

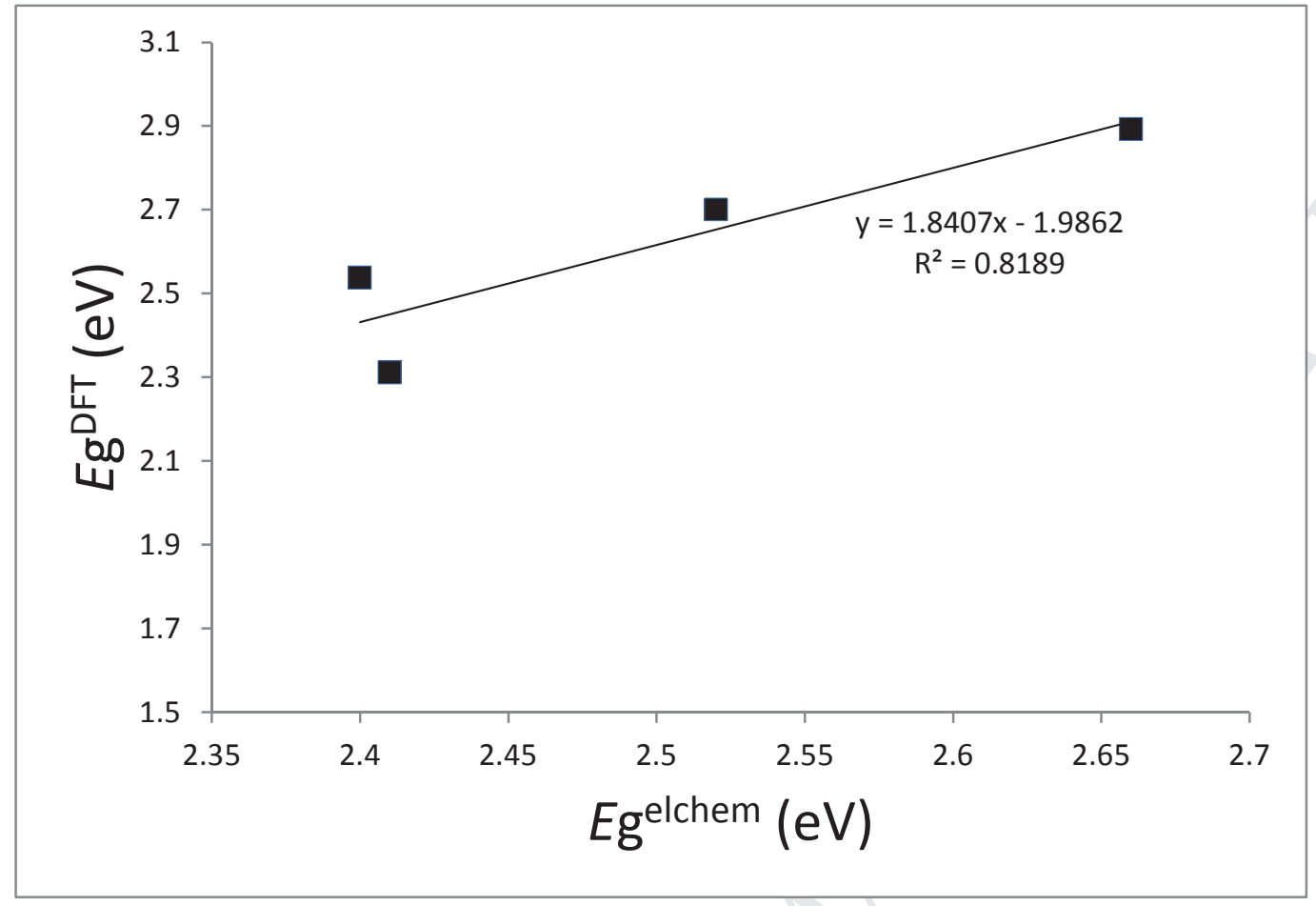

DFT calculated vs. electrochemical gaps Eg. 


\section{Chromophore 1}

Total energy $=-7.329624646 \mathrm{E}+4 \mathrm{eV}, \quad-2.693655307 \mathrm{E}+3$ hartree, $\quad-1.690276786 \mathrm{E}+6 \mathrm{kcal} / \mathrm{mol}$ Cartesian coordinates
$N$ Atom $X \quad Y \quad Z$
$\begin{array}{lllll}1 & N & 0.0000 & 0.06160 & 0.0000\end{array}$
$\begin{array}{lllll}2 & C & 1.40800 & 0.06160 & 0.12670\end{array}$
$\begin{array}{lllll}3 & C & -0.59430 & 0.06160 & -1.28270\end{array}$
$\begin{array}{lllll}4 & C & -0.81370 & 0.06160 & 1.15600\end{array}$
5 C $\quad-1.96510 \quad-0.73100 \quad 1.23600$
$\begin{array}{lllll}6 & \text { C } & 2.19990 & 0.89360 & -0.70230\end{array}$
7 C $2.05290-0.73100 \quad 1.08380$
$\begin{array}{llllll}8 & C & -1.70820 & 0.89360 & -1.55400\end{array}$
9 C $-0.08790-0.73100-2.31980$
$\begin{array}{lllll}10 & C & -0.49170 & 0.89360 & 2.25630\end{array}$
$\begin{array}{lllll}11 & C & 4.23340 & 0.06160 & 0.38230\end{array}$
$\begin{array}{lllll}12 & C & -2.44780 & 0.06160 & 3.47510\end{array}$
$\begin{array}{lllll}13 & C & -1.78560 & 0.06160 & -3.85740\end{array}$
$\begin{array}{lllll}14 & C & -1.28760 & 0.89360 & 3.38980\end{array}$
15 C $\quad-2.76750 \quad-0.73100 \quad 2.36420$
$\begin{array}{lllll}16 & C & -2.29190 & 0.89360 & -2.81000\end{array}$
17 C $\quad-0.66370 \quad-0.73100 \quad-3.57890$
$\begin{array}{lllll}18 & C & 3.57950 & 0.89360 & -0.57980\end{array}$
19 C $3.43120-0.73100 \quad 1.21460$
$\begin{array}{lllll}20 & C & 11.0813 & 0.17590 & 1.16240\end{array}$
$\begin{array}{lllll}21 & C & -6.54730 & 0.17590 & 9.01550\end{array}$
$22 \quad C \quad-4.53400 \quad 0.17590 \quad-10.1779$
$\begin{array}{lllll}23 & C & 8.25440 & 0.06160 & 0.81140\end{array}$
$\begin{array}{lllll}24 & C & -4.82990 & 0.06160 & 6.74280\end{array}$
$25 \quad C \quad-3.42450 \quad 0.06160 \quad-7.55430$ 


$$
\begin{aligned}
& \begin{array}{lllll}
26 & C & -5.35870 & 0.89360 & 8.97650
\end{array} \\
& 27 \text { C } \quad-6.84740 \quad-0.61810 \quad 7.88010 \\
& \begin{array}{lllll}
28 & C & 10.4532 & 0.89360 & 0.15260
\end{array} \\
& 29 \text { C } \quad-3.40070-0.61810-9.87010 \\
& \begin{array}{lllll}
30 & C & -5.09450 & 0.89360 & -9.12910
\end{array} \\
& 31 \text { C } \quad \begin{array}{llll}
10.2481 & -0.61810 & 1.99000
\end{array} \\
& \begin{array}{lllll}
32 & \text { C } & 9.08020 & 0.89360 & -0.02320
\end{array} \\
& 33 \quad C \quad-4.52000 \quad 0.89360 \quad 7.87530 \\
& 34 \text { C } 8.87690 \quad-0.61810 \quad 1.82260 \\
& 35 \text { C } \quad-2.86010-0.61810 \quad-8.59890 \\
& 36 \text { C }-6.01680-0.61810 \quad 6.77640 \\
& \begin{array}{lllll}
37 & C & -4.56020 & 0.89360 & -7.85200
\end{array} \\
& \begin{array}{lllll}
38 & C & 12.5250 & 0.17590 & 1.29360
\end{array} \\
& \begin{array}{lllll}
39 & C & -7.38280 & 0.17590 & 10.2002
\end{array} \\
& \begin{array}{lllll}
40 & C & -5.14220 & 0.17590 & -11.4938
\end{array} \\
& \begin{array}{lllll}
41 & C & 5.63830 & 0.06160 & 0.51790
\end{array} \\
& \begin{array}{lllll}
42 & C & -3.26770 & 0.06160 & 4.62400
\end{array} \\
& \begin{array}{lllll}
43 & C & -2.37070 & 0.06160 & -5.14190
\end{array} \\
& \begin{array}{lllll}
44 & C & -8.56590 & -0.37350 & 10.4247
\end{array} \\
& \begin{array}{lllll}
45 & C & -4.74510 & -0.37350 & -12.6306
\end{array} \\
& \begin{array}{lllll}
46 & C & 13.3110 & -0.37350 & 2.20600
\end{array} \\
& \begin{array}{lllll}
47 & C & -5.41700 & -0.17590 & -13.9148
\end{array} \\
& \begin{array}{lllll}
48 & C & -9.34210 & -0.17590 & 11.6486
\end{array} \\
& 49 \text { C } \quad 14.7591-0.17590 \quad 2.26620 \\
& \begin{array}{lllll}
50 & C & 6.84760 & 0.06160 & 0.65540
\end{array} \\
& \begin{array}{lllll}
51 & C & -2.85630 & 0.06160 & -6.25790
\end{array} \\
& \begin{array}{lllll}
52 & C & -3.99140 & 0.06160 & 5.60260
\end{array} \\
& \begin{array}{lllll}
53 & \mathrm{H} & 0.38880 & 1.52800 & 2.21630
\end{array} \\
& \begin{array}{lllll}
54 & \mathrm{H} & -2.22790 & -1.36700 & 0.40320
\end{array}
\end{aligned}
$$



$55 \quad H \quad-2.11370 \quad 1.52800 \quad-0.77150$
$56 \quad \mathrm{H} \quad 0.76470-1.36700 \quad-2.13100$
$\begin{array}{lllll}57 & \mathrm{H} & 1.72500 & 1.52800 & -1.44480\end{array}$
$58 \quad \mathrm{H} \quad 1.46320-1.36700 \quad 1.72780$
59 C $\quad-10.5680-0.89360 \quad 11.7990$
60 C $\quad-4.93420 \quad-0.89360 \quad-15.0517$
61 C $15.5022-0.89360 \quad 3.25270$
$\begin{array}{lllll}62 & \mathrm{H} & -3.14690 & 1.52800 & -2.99920\end{array}$
63 H $\quad-0.25850-1.36700 \quad-4.36390$
$64 \quad H \quad 4.17090 \quad 1.52800 \quad-1.22570$
$\begin{array}{lllll}65 & \mathrm{H} & 3.90850 & -1.36700 & 1.95810\end{array}$
$\begin{array}{lllll}66 & \mathrm{H} & -1.02400 & 1.52800 & 4.22490\end{array}$
$\begin{array}{lllll}67 & \mathrm{H} & -3.65000 & -1.36700 & 2.40580\end{array}$
68 C $\quad-5.62200-0.73100-16.2435$
69 C $\quad-11.2562-0.73100 \quad 12.9905$
$\begin{array}{lllll}70 & C & 16.8783 & -0.73100 & 3.25290\end{array}$
$\begin{array}{lllll}71 & N & -8.87700 & 0.61810 & 12.6483\end{array}$
$\begin{array}{lllll}72 & N & -6.51530 & 0.61810 & -14.0119\end{array}$
$\begin{array}{lllll}73 & N & 15.3923 & 0.61810 & 1.36350\end{array}$
$\begin{array}{lllll}74 & \text { C } & -9.62670 & 0.61810 & 13.7390\end{array}$
$\begin{array}{lllll}75 & C & 16.7116 & 0.61810 & 1.46750\end{array}$
$\begin{array}{lllll}76 & C & -7.08490 & 0.61810 & -15.2065\end{array}$
$\begin{array}{lllll}77 \quad H & 10.7354 & -1.24610 & 2.74960\end{array}$
$78 \quad H \quad 11.0662 \quad 1.52800 \quad-0.50520$
$\begin{array}{lllll}79 & \mathrm{H} & -5.09560 & 1.52800 & 9.83620\end{array}$
$80 \quad$ H $\quad-7.74900 \quad-1.24610 \quad 7.92230$
$81 \quad H \quad-5.97060 \quad 1.52800 \quad-9.33100$
82 H $\quad-2.98650-1.24610-10.6720$
$83 \quad$ H $\quad 8.30490 \quad-1.36700 \quad 2.47770$ 

$84 \quad H \quad-2.00670 \quad-1.36700 \quad-8.43110$
$\begin{array}{lllll}85 & \text { H } & -5.01460 & 1.52800 & -7.06690\end{array}$
$86 \quad$ H $\quad 8.62740 \quad 1.52800 \quad-0.80940$
$\begin{array}{lllll}87 & \mathrm{H} & -3.61280 & 1.52800 & 7.87620\end{array}$
$88 \quad H \quad-6.29820 \quad-1.36700 \quad 5.95340$
$\begin{array}{lllll}89 & H & -6.02420 & 0.89360 & -11.5724\end{array}$
$\begin{array}{lllll}90 & \mathrm{H} & 13.0341 & 0.89360 & 0.56910\end{array}$
$\begin{array}{lllll}91 & H & -7.00990 & 0.89360 & 11.0033\end{array}$
$92 \quad \mathrm{H} \quad-9.00580-1.01850 \quad 9.66740$
$93 \quad$ H $\quad 12.8751-1.01850 \quad 2.96550$
$94 \quad \mathrm{H} \quad-3.86930-1.01850 \quad-12.6329$
$\begin{array}{lllll}95 & N & 17.5107 & 0.06160 & 2.36270\end{array}$
$\begin{array}{lllll}96 & N & -6.70920 & 0.06160 & -16.3461\end{array}$
$\begin{array}{lllll}97 & N & -10.8015 & 0.06160 & 13.9834\end{array}$
$98 \quad H \quad-10.9667 \quad-1.52800 \quad 11.0113$
$99 \quad \mathrm{H} \quad-4.05270 \quad-1.52800 \quad-15.0031$

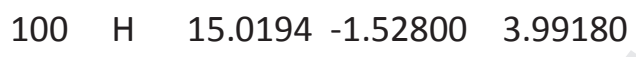
$\begin{array}{lllll}101 & \text { H } & -12.2125 & -1.24610 & 13.1621\end{array}$
102 H $\quad-5.29250-1.24610 \quad-17.1574$
103 H $17.5049-1.24610 \quad 3.99530$
$\begin{array}{lllll}104 & H & -9.23760 & 1.24610 & 14.5368\end{array}$
$\begin{array}{lllll}105 & \text { H } & -7.97040 & 1.24610 & -15.2684\end{array}$
$\begin{array}{lllll}106 & \text { H } & 17.2080 & 1.24610 & 0.73160\end{array}$ 


\section{Chromophore 2}

Total energy $=-8.584017972 \mathrm{E}+4 \mathrm{eV}, \quad-3.154647978 \mathrm{E}+3$ hartree, $-1.979551070 \mathrm{E}+6 \mathrm{kcal} / \mathrm{mol}$ Cartesian coordinates
$N$ Atom $X \quad Y \quad Z$
1 C $11.1057-0.42310 \quad 0.78000$
2 C $\quad-6.22840-0.42310 \quad 9.22780$
3 C $\quad-4.87730 \quad-0.42310-10.0079$

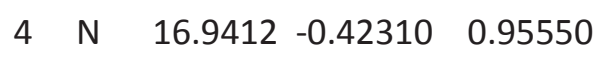
$5 \quad \mathrm{~N} \quad-7.64310-0.42310-15.1492$

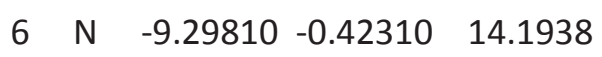
$\begin{array}{lllll}7 & C & -8.91120 & 0.31700 & 11.9341\end{array}$
$\begin{array}{lllll}8 & C & -5.87970 & 0.31700 & -13.6844\end{array}$
$9 \quad$ C $\quad 14.7909 \quad 0.31700 \quad 1.75030$
10 C $8.26830 \quad-0.54890 \quad 0.56070$
11 C $-4.61970-0.54890 \quad 6.88020$
12 C $\quad-3.64850-0.54890 \quad-7.44090$
$\begin{array}{lllll}13 & \mathrm{H} & -4.51710 & 2.54710 & -16.6243\end{array}$
$14 \quad \mathrm{H} \quad 16.6556 \quad 2.54710 \quad 4.40020$
$\begin{array}{lllll}15 & H & -12.1385 & 2.54710 & 12.2241\end{array}$
$\begin{array}{lllll}16 & H & -4.84470 & -1.88800 & 9.99410\end{array}$
$\begin{array}{lllll}17 & H & -7.37630 & 1.04330 & 8.10940\end{array}$
$18 \quad H \quad-6.23280-1.88800 \quad-9.19270$
$\begin{array}{lllll}19 & \mathrm{H} & -3.33480 & 1.04330 & -10.4428\end{array}$
$20 \quad \mathrm{H} \quad 10.7111 \quad 1.04330 \quad 2.33340$
$21 \quad$ H $\quad 11.0775-1.88800 \quad-0.80140$
$22 \quad \mathrm{H} \quad-5.58190 \quad 2.54710 \quad-18.8718$
$\begin{array}{lllll}23 & \mathrm{H} & -13.5525 & 2.54710 & 14.2700\end{array}$
$24 \quad \mathrm{H} \quad 19.1344 \quad 2.54710 \quad 4.60180$
25 C $-1.73960-1.46570-1.53310$ 


$$
\begin{aligned}
& 26 \text { C } 2.19750-1.46570-0.73990 \\
& 27 \text { C } \quad-0.13360 \quad 0.16980 \quad-2.29840 \\
& \begin{array}{lllll}
28 & C & 2.05720 & 0.16980 & 1.03350
\end{array} \\
& 29 \text { C } \quad-1.92370 \quad 0.16980 \quad 1.26480 \\
& 30 \text { C } \quad-0.45800 \quad-1.46570 \quad 2.27310 \\
& \begin{array}{lllll}
31 & C & -5.21410 & 0.31700 & -12.3922
\end{array} \\
& \begin{array}{lllll}
32 & \mathrm{C} & 13.3390 & 0.31700 & 1.68060
\end{array} \\
& \begin{array}{lllll}
33 & \mathrm{C} & -8.12490 & 0.31700 & 10.7116
\end{array} \\
& 34 \text { C } \quad-4.31810 \quad-1.29320 \quad 8.00440 \\
& \begin{array}{lllll}
35 & C & -5.74490 & 0.35230 & 6.95850
\end{array} \\
& 36 \text { C } \quad-4.77290-1.29320-7.74180 \\
& \begin{array}{lllll}
37 & C & -3.15380 & 0.35230 & -8.45450
\end{array} \\
& 38 \text { C } 9.09100 \quad-1.29320-0.26260 \\
& \begin{array}{lllll}
39 & \mathrm{C} & 8.89870 & 0.35230 & 1.49600
\end{array} \\
& \begin{array}{lllll}
40 & \text { H } & 15.2029 & -1.04330 & 0.12680
\end{array} \\
& \begin{array}{lllll}
41 & \mathrm{H} & -7.71120 & -1.04330 & 13.1027
\end{array} \\
& 42 \text { H } \quad-7.49170-1.04330-13.2295 \\
& \begin{array}{lllll}
43 & C & -5.93860 & 1.04330 & -15.8298
\end{array} \\
& \begin{array}{lllll}
44 & C & -10.7397 & 1.04330 & 13.0579
\end{array} \\
& \begin{array}{lllll}
45 & C & 16.6783 & 1.04330 & 2.77200
\end{array} \\
& 46 \text { C } \quad-0.79040 \quad-0.67560 \quad 1.17190 \\
& 47 \text { C } \quad-0.61970-0.67560-1.27050 \\
& 48 \text { C } \quad 1.41010-0.67560 \quad 0.09860 \\
& 49 \text { C } \quad-3.03410-0.54890-6.17250 \\
& 50 \text { C } \quad-3.82850-0.54890 \quad 5.71390 \\
& 51 \text { C } 6.86260-0.54890 \quad 0.45860 \\
& \begin{array}{lllll}
52 & \mathrm{C} & -11.2128 & 0.42310 & 15.3576
\end{array} \\
& \begin{array}{lllll}
53 & \mathrm{C} & 18.9065 & 0.42310 & 2.03180
\end{array} \\
& \begin{array}{lllll}
54 & C & -7.69370 & 0.42310 & -17.3894
\end{array}
\end{aligned}
$$




$$
\begin{aligned}
& \begin{array}{lllll}
55 & \mathrm{H} & 3.91870 & 0.82250 & 1.85000
\end{array} \\
& \begin{array}{lllll}
56 & \mathrm{H} & -0.35720 & 0.82250 & -4.31870
\end{array} \\
& \begin{array}{lllll}
57 & \mathrm{H} & -3.21550 & -2.10960 & -2.95710
\end{array} \\
& \begin{array}{lllll}
58 & H & -3.56150 & 0.82250 & 2.46860
\end{array} \\
& 59 \text { H } 4.16870-2.10960-1.30620 \\
& 60 \quad H \quad-0.95320-2.10960 \quad 4.26330 \\
& \begin{array}{lllll}
61 & C & -8.58860 & -0.42310 & 13.0954
\end{array} \\
& 62 \text { C } 15.6352-0.42310 \quad 0.89020 \\
& 63 \text { C } \quad-7.04670-0.42310-13.9856 \\
& 64 \text { C } \quad-5.10340-1.29320 \quad 9.14240 \\
& 65 \text { C } \quad-6.52320 \quad 0.42310 \quad 8.09840 \\
& 66 \text { C } \quad-3.75180 \quad 0.42310 \quad-9.69840 \\
& \begin{array}{lllll}
67 & C & 10.2750 & 0.42310 & 1.60010
\end{array} \\
& 68 \text { C } \quad 10.4692-1.29320-0.15150 \\
& 69 \text { C } \quad-5.36580-1.29320-8.99090 \\
& \begin{array}{lllll}
70 & C & 17.2944 & 1.88800 & 3.74760
\end{array} \\
& \begin{array}{lllll}
71 & C & -5.40170 & 1.88800 & -16.8512
\end{array} \\
& \begin{array}{lllll}
72 & C & -11.8927 & 1.88800 & 13.1036
\end{array} \\
& \begin{array}{lllll}
73 & C & -7.10280 & 0.42310 & -16.1093
\end{array} \\
& \begin{array}{lllll}
74 & C & -10.3997 & 0.42310 & 14.2059
\end{array} \\
& \begin{array}{lllll}
75 & C & 17.5025 & 0.42310 & 1.90350
\end{array} \\
& \begin{array}{lllll}
76 & N & -5.34520 & 1.04330 & -14.6141
\end{array} \\
& \begin{array}{lllll}
77 & N & 15.3288 & 1.04330 & 2.67800
\end{array} \\
& \begin{array}{lllll}
78 & N & -9.98360 & 1.04330 & 11.9361
\end{array} \\
& \begin{array}{lllll}
79 & \text { H } & 13.0347 & -1.04330 & 0.12860
\end{array} \\
& 80 \quad \mathrm{H} \quad-6.62870 \quad-1.04330 \quad 11.2241 \\
& 81 \quad H \quad-6.40600 \quad-1.04330-11.3527 \\
& \begin{array}{lllll}
82 & \mathrm{C} & -12.3282 & 1.29320 & 15.3721
\end{array} \\
& \begin{array}{lllll}
83 & \mathrm{C} & 19.4767 & 1.29320 & 2.99050
\end{array}
\end{aligned}
$$




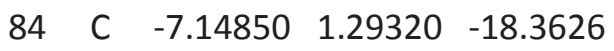

$$
\begin{aligned}
& \begin{array}{lllll}
85 & N & 0.0000 & -0.67560 & 0.0000
\end{array} \\
& \begin{array}{lllll}
86 & \mathrm{H} & 0.40830 & -2.10960 & 2.21940
\end{array} \\
& \begin{array}{lllll}
87 & H & -2.20640 & 0.82250 & 0.43310
\end{array} \\
& \begin{array}{lllll}
88 & \mathrm{H} & 0.72810 & 0.82250 & -2.12730
\end{array} \\
& 89 \quad \mathrm{H} \quad 1.71790-2.10960-1.46330 \\
& \begin{array}{lllll}
90 & \mathrm{H} & 1.47820 & 0.82250 & 1.69430
\end{array} \\
& 91 \quad \mathrm{H} \quad-2.12620-2.10960 \quad-0.75610 \\
& \begin{array}{lllll}
92 & C & -2.69500 & 0.16980 & 2.41410
\end{array} \\
& \begin{array}{lllll}
93 & C & 3.43820 & 0.16980 & 1.12690
\end{array} \\
& 94 \text { C } 3.57900-1.46570-0.65290 \\
& 95 \text { C } \quad-0.74310 \quad 0.16980 \quad-3.54100 \\
& 96 \text { C } \quad-2.35490-1.46570 \quad-2.77310
\end{aligned}
$$

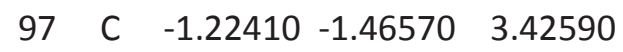

$$
\begin{aligned}
& \begin{array}{llllll}
98 & \mathrm{H} & -7.60440 & 1.29320 & -19.3482
\end{array} \\
& \begin{array}{lllll}
99 & \mathrm{H} & -12.9538 & 1.29320 & 16.2597
\end{array} \\
& \begin{array}{lllll}
100 & \mathrm{H} & 20.5582 & 1.29320 & 3.08850
\end{array} \\
& 101 \text { C } \quad-5.54700 \quad-0.42310-11.2970 \\
& 102 \text { C } 12.5570-0.42310 \quad 0.84460 \\
& \begin{array}{lllll}
103 & C & -7.01000 & -0.42310 & 10.4523
\end{array} \\
& 104 \quad H \quad-10.9332-0.16980 \quad 16.2168 \\
& \begin{array}{lllll}
105 & \text { H } & -8.57760 & -0.16980 & -17.5769
\end{array} \\
& \begin{array}{lllll}
106 & H & 19.5108 & -0.16980 & 1.36000
\end{array} \\
& \begin{array}{lllll}
107 & C & -12.6681 & 1.99770 & 14.2403
\end{array} \\
& \begin{array}{lllll}
108 & C & 18.6665 & 1.99770 & 3.85080
\end{array} \\
& \begin{array}{lllll}
109 & C & -5.99840 & 1.99770 & -18.0911
\end{array} \\
& 110 \text { C } \quad-1.87270-0.54890-3.81130 \\
& 111 \text { C } 4.23700 \quad-0.54890 \quad 0.28380 \\
& 112 \text { C } \quad-2.36430 \quad-0.54890 \quad 3.52740
\end{aligned}
$$



113 C $5.64470-0.54890 \quad 0.37510$
114 C $\quad-3.14720 \quad-0.54890 \quad 4.70090$
115 C $\quad-2.49750 \quad-0.54890 \quad-5.07600$
$\begin{array}{lllll}116 & \mathrm{H} & 12.8862 & 1.04330 & 2.40900\end{array}$
$\begin{array}{lllll}117 & \mathrm{H} & -4.35690 & 1.04330 & -12.3643\end{array}$
$\begin{array}{lllll}118 & H & -8.52930 & 1.04330 & 9.95530\end{array}$
119 H $8.63270-1.92460 \quad-0.99140$
$120 \quad \mathrm{H} \quad-2.30470 \quad 1.04330 \quad-8.30820$
$\begin{array}{lllll}121 & \mathrm{H} & 8.34750 & 1.04330 & 2.15820\end{array}$
$\begin{array}{lllll}122 & H & -6.04280 & 1.04330 & 6.15000\end{array}$
123 H $\quad-5.17490 \quad-1.92460 \quad-6.98050$
$124 \quad H \quad-3.45780-1.92460 \quad 7.97180$ 


\section{Chromophore 3}

Total energy $=-9.947637839 \mathrm{E}+4 \mathrm{eV},-3.655781674 \mathrm{E}+3$ hartree, $-2.294013968 \mathrm{E}+6 \mathrm{kcal} / \mathrm{mol}$ Cartesian coordinates
$\begin{array}{lllll}N & \text { Atom } & X & Y & Z\end{array}$
$\begin{array}{lllll}1 & N & 0.0000 & -0.50690 & 0.0000\end{array}$
2 C $1.04530-0.50690-0.94970$
$\begin{array}{lllll}3 & C & 0.29980 & -0.50690 & 1.38010\end{array}$
4 C $\quad-1.34510 \quad-0.50690 \quad-0.43040$
5 C C $0.97380 \quad 0.30800 \quad-2.10120$
$\begin{array}{lllll}6 & C & 1.33280 & 0.30800 & 1.89390\end{array}$
7 C $2.17700-1.32090-0.77660$
$\begin{array}{lllll}8 & C & -0.41590 & -1.32090 & 2.27360\end{array}$
$\begin{array}{llllll}9 & C & -2.30660 & 0.30800 & 0.20730\end{array}$
10 C $\quad-1.76100 \quad-1.32090 \quad-1.49700$
11 C $0.90770-0.50690 \quad 4.14720$
12 C $3.13770-0.50690 \quad-2.85970$
13 C $\quad-4.04540-0.50690-1.28750$
14 C $-3.07270-1.32090-1.92000$
$\begin{array}{lllll}15 & C & 1.64190 & 0.30800 & 3.24590\end{array}$
$\begin{array}{lllll}16 & C & -0.12650 & -1.32090 & 3.62100\end{array}$
$\begin{array}{lllll}17 & C & 1.99010 & 0.30800 & -3.04490\end{array}$
$\begin{array}{lllll}18 & C & -3.63200 & 0.30800 & -0.20110\end{array}$
19 C $3.19910-1.32090-1.70100$
20 C $\quad-10.4010-0.30800 \quad-2.59950$
21 C $2.94930-0.30800 \quad 10.3072$
22 C $7.45170-0.30800 \quad-7.70780$
23 C $6.09540-0.50690 \quad-5.60620$
24 C $\quad-7.90280 \quad-0.50690 \quad-2.47570$
25 C $1.80740-0.50690 \quad 8.08190$ 


$$
\begin{aligned}
& 26 \text { C } 8.08080-1.08190-6.69290 \\
& 27 \text { C } \quad 1.75580-1.08190 \quad 10.3447 \\
& 28 \text { C } \quad-9.83660-1.08190 \quad-3.65180 \\
& 29 \text { C } 8.01400-0.00110 \quad-8.96740 \\
& 30 \text { C } \quad-11.7730-0.00110-2.45670 \\
& 31 \text { C } 3.75900 \quad-0.00110 \quad 11.4240 \\
& 32 \text { C } \quad-8.45020-1.32090-3.56230 \\
& 33 \text { C } 7.31020-1.32090 \quad-5.53700 \\
& 34 \text { C } 1.14010-1.32090 \quad 9.09930 \\
& 35 \text { C } 1.21890-0.50690 \quad 5.52050 \\
& 36 \text { C } 4.17140-0.50690-3.81580 \\
& 37 \text { C } \quad-5.39030-0.50690-1.70460 \\
& \begin{array}{lllll}
38 & S & -9.13540 & 0.17990 & -1.52360
\end{array} \\
& \begin{array}{lllll}
39 & \mathrm{~S} & 5.88720 & 0.17990 & -7.14970
\end{array} \\
& \begin{array}{lllll}
40 & S & 3.24820 & 0.17990 & 8.67330
\end{array} \\
& \begin{array}{lllll}
41 & C & 4.91780 & 0.50690 & 11.4192
\end{array} \\
& 42 \text { C } 7.43040 \quad 0.50690 \quad-9.96850 \\
& 43 \text { C } \quad-12.3482 \quad 0.50690 \quad-1.45060 \\
& \begin{array}{lllll}
44 & C & 8.00360 & 1.08190 & -11.2685
\end{array} \\
& \begin{array}{lllll}
45 & C & 5.75700 & 1.08190 & 12.5656
\end{array} \\
& 46 \text { C } \quad-13.7606 \quad 1.08190 \quad-1.29710 \\
& 47 \text { C } 5.05730-0.50690-4.65840 \\
& 48 \text { C } 1.50560-0.50690 \quad 6.70900 \\
& 49 \text { C } \quad-6.56300-0.50690-2.05060 \\
& \begin{array}{lllll}
50 & \mathrm{H} & 1.93090 & 1.08190 & 1.21720
\end{array} \\
& \begin{array}{lllll}
51 & \mathrm{H} & -1.22960 & -1.98330 & 1.90980
\end{array} \\
& \begin{array}{lllll}
52 & \mathrm{H} & -1.03910 & -1.98330 & -2.01980
\end{array} \\
& \begin{array}{lllll}
53 & \mathrm{H} & 2.26880 & -1.98330 & 0.11000
\end{array} \\
& \begin{array}{lllll}
54 & \mathrm{H} & -2.01950 & 1.08190 & 1.06360
\end{array}
\end{aligned}
$$



$\begin{array}{lllll}55 & \mathrm{H} & 0.08860 & 1.08190 & -2.28080\end{array}$
$\begin{array}{lllll}56 & \mathrm{H} & -3.37530 & -1.98330 & -2.75080\end{array}$
$\begin{array}{lllll}57 & H & -4.35960 & 0.97400 & 0.30190\end{array}$
$\begin{array}{lllll}58 & \mathrm{H} & -0.69460 & -1.98330 & 4.29850\end{array}$
$\begin{array}{lllll}59 & \mathrm{H} & 2.44120 & 0.97400 & 3.62460\end{array}$
$60 \quad H \quad 4.06990-1.98330-1.54770$
$\begin{array}{lllll}61 & \mathrm{H} & 1.91840 & 0.97400 & -3.92640\end{array}$
62 C $\quad-14.7706 \quad 0.50690 \quad-2.20340$
$\begin{array}{lllll}63 & C & 5.47710 & 0.50690 & 13.8935\end{array}$
$\begin{array}{lllll}64 & C & 9.29360 & 0.50690 & -11.6900\end{array}$
65 C $9.67980 \quad 1.08190 \quad-12.9683$
$\begin{array}{lllll}66 & C & 6.39100 & 1.08190 & 14.8671\end{array}$
$\begin{array}{lllll}67 & C & -16.0708 & 1.08190 & -1.89880\end{array}$
$\begin{array}{lllll}68 & N & -14.0856 & 1.68570 & -0.18160\end{array}$
$\begin{array}{lllll}69 & N & 7.20000 & 1.68570 & -12.1077\end{array}$
$\begin{array}{lllll}70 & N & 6.88550 & 1.68570 & 12.2893\end{array}$
$\begin{array}{lllll}71 & C & -15.3695 & 1.98330 & -0.00440\end{array}$
72 C $7.68850 \quad 1.98330 \quad-13.3082$
$\begin{array}{lllll}73 & C & 7.68090 & 1.98330 & 13.3126\end{array}$
74 H $\quad-10.4490 \quad-1.50800-4.44160$
$\begin{array}{lllll}75 & \text { H } & 1.37800 & -1.50800 & 11.2699\end{array}$
76 H $9.07100-1.50800-6.82830$
$77 \quad$ H $\quad 7.62470-1.68570-4.66120$
$\begin{array}{lllll}78 & \mathrm{H} & 0.22430 & -1.68570 & 8.93380\end{array}$
79 H $\quad-7.84900-1.68570 \quad-4.27260$
$80 \quad \mathrm{H} \quad-12.4041-0.50690 \quad-3.24540$
81 H $9.01260-0.50690-9.11950$
$\begin{array}{lllll}82 & \text { H } & 3.39140 & -0.50690 & 12.3649\end{array}$
$83 \quad N \quad-16.3999 \quad 1.68570 \quad-0.79580$ 

$\begin{array}{lllll}84 & N & 8.88910 & 1.68570 & -13.8048\end{array}$
$\begin{array}{lllll}85 & N & 7.51080 & 1.68570 & 14.6006\end{array}$
86 H $\quad-11.7344 \quad 1.08190 \quad-0.64480$
$\begin{array}{lllll}87 & \mathrm{H} & 6.42560 & 1.08190 & -9.83990\end{array}$
$\begin{array}{lllll}88 & \mathrm{H} & 5.30870 & 1.08190 & 10.4847\end{array}$
89 H $\quad-14.5606-0.00110-3.11380$
$90 \quad H \quad 9.97690-0.00110-11.0529$
$91 \quad H \quad 4.58360-0.00110 \quad 14.1667$
$92 \quad \mathrm{H} \quad-16.8916 \quad 0.73260 \quad-2.56600$
$93 \quad \mathrm{H} \quad 10.6680 \quad 0.73260 \quad-13.3455$
$94 \quad \mathrm{H} \quad 6.22350 \quad 0.73260 \quad 15.9116$
$95 \quad \mathrm{H} \quad-15.6099 \quad 2.53740 \quad 0.90260$
$96 \quad \mathrm{H} \quad 7.02330 \quad 2.53740 \quad-13.9699$
$97 \quad \mathrm{H} \quad 8.58660 \quad 2.53740 \quad 13.0673$ 


\section{Chromophore 4}

Total energy $=-1.120198022 \mathrm{E}+5 \mathrm{eV}, \quad-4.116755622 \mathrm{E}+3$ hartree, $\quad-2.583276503 \mathrm{E}+6 \mathrm{kcal} / \mathrm{mol}$ Cartesian coordinates
$\begin{array}{lllll}N & \text { Atom } & X & Y & Z\end{array}$

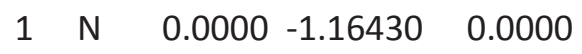
2 C $1.14620-1.16430 \quad 0.82820$
$\begin{array}{lllll}3 & C & -1.29040 & -1.16430 & 0.57860\end{array}$
4 C $0.14410 \quad-1.16430 \quad-1.40680$
$\begin{array}{lllll}5 & C & -2.31570 & -1.97870 & 0.06380\end{array}$
$\begin{array}{lllll}6 & \text { C } & -1.61740 & -0.30290 & 1.65140\end{array}$
$\begin{array}{lllll}7 & C & -0.62150 & -0.30290 & -2.22640\end{array}$
8 C $2.23890-0.30290 \quad 0.57500$
9 C $1.10260-1.97870-2.03740$
10 C $1.21310-1.97870 \quad 1.97360$
11 C $\quad-3.90170-1.16430 \quad 1.67460$
12 C $3.40100-1.16430 \quad 2.54170$
13 C $0.50060-1.16430-4.21620$
14 C $\quad-3.59060-1.97870 \quad 0.56530$
15 C $\quad-0.47930-0.30290-3.59280$
16 C $1.30570-1.97870-3.39220$
$\begin{array}{lllll}17 & C & 3.35110 & -0.30290 & 1.38130\end{array}$
$\begin{array}{lllll}18 & \text { C } & 2.28490 & -1.97870 & 2.82690\end{array}$
$\begin{array}{lllll}19 & C & -2.87180 & -0.30290 & 2.21150\end{array}$
20 C $\quad \begin{array}{llll}14.0756 & 0.99150 & 7.51610\end{array}$
$\begin{array}{lllll}21 & C & -13.5470 & 0.99150 & 8.43170\end{array}$
$\begin{array}{lllll}22 & C & -0.52860 & 0.99150 & -15.9479\end{array}$
23 C $14.8075 \quad 1.97870 \quad 6.66850$
$\begin{array}{lllll}24 & C & -13.1789 & 1.97870 & 9.48940\end{array}$
$\begin{array}{lllll}25 & C & -1.62860 & 1.97870 & -16.1580\end{array}$ 


$$
\begin{aligned}
& 26 \quad C \quad-11.4100 \quad 1.16430 \quad 7.61360 \\
& \begin{array}{lllll}
27 & C & -0.88860 & 1.16430 & -13.6881
\end{array} \\
& \begin{array}{lllll}
28 & C & 12.2985 & 1.16430 & 6.07450
\end{array} \\
& 29 \text { C } \quad-9.65210 \quad-0.55000 \quad 4.63510 \\
& 30 \text { C } 8.84010-0.55000 \quad 6.04150 \\
& 31 \text { C } 0.81200 \quad-0.55000-10.6765 \\
& \begin{array}{lllll}
32 & C & -14.8756 & 0.55000 & 8.37690
\end{array} \\
& 33 \text { C } \quad 14.6924 \quad 0.55000 \quad 8.69420 \\
& 34 \text { C } \quad 0.18320 \quad 0.55000 \quad-17.0711 \\
& \begin{array}{lllll}
35 & C & -10.3847 & 0.67290 & 6.66320
\end{array} \\
& \begin{array}{lllll}
36 & C & -0.57810 & 0.67290 & -12.3250
\end{array} \\
& \begin{array}{lllll}
37 & C & 10.9628 & 0.67290 & 5.66180
\end{array} \\
& \begin{array}{lllll}
38 & C & -1.89710 & 2.33220 & -17.4807
\end{array} \\
& \begin{array}{lllll}
39 & C & -14.1902 & 2.33220 & 10.3833
\end{array} \\
& \begin{array}{lllll}
40 & \mathrm{C} & 16.0873 & 2.33220 & 7.09740
\end{array} \\
& \begin{array}{lllll}
41 & N & -12.6648 & 0.55000 & 7.48380
\end{array} \\
& \begin{array}{lllll}
42 & \mathrm{~N} & 12.8135 & 0.55000 & 7.22620
\end{array} \\
& 43 \quad N \quad-0.14870 \quad 0.55000 \quad-14.7099 \\
& \begin{array}{lllll}
44 & C & -11.0789 & 1.97870 & 8.65070
\end{array} \\
& \begin{array}{lllll}
45 & C & -1.95230 & 1.97870 & -13.9199
\end{array} \\
& \begin{array}{lllll}
46 & C & 13.0311 & 1.97870 & 5.26930
\end{array} \\
& 47 \text { C } 0.40990-0.06940-11.9769 \\
& \begin{array}{lllll}
48 & \text { C } & 10.1674 & -0.06940 & 6.34350
\end{array} \\
& 49 \text { C } \quad-10.5773 \quad-0.06940 \quad 5.63350 \\
& 50 \text { C } 0.99060-1.16430 \quad-8.20960 \\
& 51 \text { C } 6.61450-1.16430 \quad 4.96270 \\
& 52 \text { C } \quad-7.60510-1.16430 \quad 3.24690 \\
& \begin{array}{lllll}
53 & \mathrm{C} & 16.6687 & 1.82600 & 8.21900
\end{array} \\
& 54 \text { C } \quad-1.21650 \quad 1.82600 \quad-18.5450
\end{aligned}
$$




$$
\begin{aligned}
& 55 \text { C } \quad-15.4522 \quad 1.82600 \quad 10.3260 \\
& 56 \text { C } \quad-9.86690 \quad-1.51910 \quad 3.62310 \\
& 57 \text { C } 8.07120-1.51910 \quad 6.73340 \\
& 58 \text { C } \quad 1.79570-1.51910-10.3566 \\
& \begin{array}{lllll}
59 & \text { C } & 15.9688 & 0.99150 & 9.02470
\end{array} \\
& \begin{array}{lllll}
60 & C & -15.8000 & 0.99150 & 9.31710
\end{array} \\
& \begin{array}{lllll}
61 & C & -0.16880 & 0.99150 & -18.3417
\end{array} \\
& \begin{array}{lllll}
62 & \mathrm{~N} & 14.2443 & 2.33220 & 5.53750
\end{array} \\
& \begin{array}{lllll}
63 & N & -11.9178 & 2.33220 & 9.56720
\end{array} \\
& \begin{array}{lllll}
64 & N & -2.32650 & 2.33220 & -15.1047
\end{array} \\
& 65 \text { C } \quad-8.75150-1.97870 \quad 2.82280 \\
& 66 \text { C } 6.82040-1.97870 \quad 6.16760 \\
& 67 \text { C } 1.93110-1.97870 \quad-8.99040 \\
& 68 \quad \text { C } 4.52590-1.16430 \quad 3.38830 \\
& 69 \text { C } \quad-5.19730-1.16430 \quad 2.22540 \\
& 70 \text { C } 0.67140-1.16430 \quad-5.61370 \\
& 71 \text { S } \quad-0.07870 \quad-0.19630 \quad-9.21040 \\
& \begin{array}{lllll}
72 & S & -7.93710 & -0.19630 & 4.67340
\end{array} \\
& \begin{array}{lllll}
73 & S & 8.01580 & -0.19630 & 4.53700
\end{array} \\
& 74 \text { C } 0.82680-1.16430-6.82680 \\
& 75 \text { C } 5.49880-1.16430 \quad 4.12940 \\
& \begin{array}{lllll}
76 & C & -6.32560 & -1.16430 & 2.69740
\end{array} \\
& \begin{array}{lllll}
77 & \mathrm{H} & -2.08550 & -2.74860 & -0.80490
\end{array} \\
& \begin{array}{lllll}
78 & \mathrm{H} & 0.34570 & -2.74860 & 2.20850
\end{array} \\
& \begin{array}{lllll}
79 & \text { H } & 2.22150 & 0.41640 & -0.30990
\end{array} \\
& \begin{array}{lllll}
80 & \mathrm{H} & -0.84230 & 0.41640 & 2.07880
\end{array} \\
& 81 \quad \mathrm{H} \quad 1.73980-2.74860-1.40370 \\
& 82 \text { H } \quad-1.37910 \quad 0.41640 \quad-1.76890 \\
& \begin{array}{lllll}
83 & \mathrm{H} & -3.09410 & 0.55000 & 3.05030
\end{array}
\end{aligned}
$$




$$
\begin{aligned}
& \begin{array}{lllll}
84 & \mathrm{H} & 4.18870 & 0.55000 & 1.15440
\end{array} \\
& 85 \quad H \quad 2.29760-2.74860 \quad 3.70390 \\
& \begin{array}{lllll}
86 & H & -1.09460 & 0.55000 & -4.20480
\end{array} \\
& \begin{array}{lllll}
87 & H & -4.35650 & -2.74860 & 0.13780
\end{array} \\
& \begin{array}{lllll}
88 & \mathrm{H} & 2.05890 & -2.74860 & -3.84180
\end{array} \\
& \begin{array}{lllll}
89 & \mathrm{H} & -13.8896 & 3.02300 & 11.1677
\end{array} \\
& 90 \quad H \quad-2.72670 \quad 3.02300 \quad-17.6126 \\
& 91 \quad \mathrm{H} \quad 16.6163 \quad 3.02300 \quad 6.44490 \\
& \begin{array}{lllll}
92 & H & -15.1298 & -0.06940 & 7.58690
\end{array} \\
& \begin{array}{lllll}
93 & \mathrm{H} & 14.1353 & -0.06940 & 9.30930
\end{array} \\
& 94 \quad \mathrm{H} \quad 0.99450 \quad-0.06940-16.8962 \\
& \begin{array}{lllll}
95 & \mathrm{H} & 10.6099 & 1.16430 & 4.73030
\end{array} \\
& 96 \quad \mathrm{H} \quad-9.40150 \quad 1.16430 \quad 6.82330 \\
& 97 \quad H \quad-1.20840 \quad 1.16430 \quad-11.5536 \\
& 98 \quad \mathrm{H} \quad 1.00810-0.55000 \quad-12.7830 \\
& 99 \quad \mathrm{H} \quad 10.5664 \quad-0.55000 \quad 7.26450 \\
& 100 \quad H \quad-11.5745 \quad-0.55000 \quad 5.51850 \\
& \begin{array}{lllll}
101 & H & -10.0638 & 2.33220 & 8.71590
\end{array} \\
& \begin{array}{lllll}
102 & H & 12.5801 & 2.33220 & 4.35760
\end{array} \\
& \begin{array}{lllll}
103 & H & -2.51630 & 2.33220 & -13.0735
\end{array} \\
& \begin{array}{lllll}
104 & H & 16.4286 & 0.55000 & 9.93750
\end{array} \\
& \begin{array}{lllll}
105 & H & -16.8204 & 0.55000 & 9.25890
\end{array} \\
& \begin{array}{lllll}
106 & \text { H } & 0.39180 & 0.55000 & -19.1963
\end{array} \\
& 107 \quad H \quad 2.42420-1.97870-11.1247 \\
& \begin{array}{lllll}
108 & \text { H } & -10.8464 & -1.97870 & 3.46300
\end{array} \\
& 109 \text { H } \quad 8.42220 \quad-1.97870 \quad 7.66170 \\
& \begin{array}{lllll}
110 & H & -16.2005 & 2.13890 & 11.0510
\end{array} \\
& 111 \quad H \quad-1.47020 \quad 2.13890-19.5555 \\
& \begin{array}{lllll}
112 & H & 17.6707 & 2.13890 & 8.50460
\end{array}
\end{aligned}
$$


$\begin{array}{lllll}113 & H & 6.08000 & -2.33220 & 6.59280\end{array}$

$114 \quad H \quad-8.74950-2.33220 \quad 1.96900$

$\begin{array}{lllll}115 & \mathrm{H} & 2.66950 & -2.33220 & -8.56180\end{array}$ 


\section{Chromophore 5}

Total energy $=-7.328964419 \mathrm{E}+4 \mathrm{eV}, \quad-2.693412672 \mathrm{E}+3$ hartree, $-1.690124532 \mathrm{E}+6 \mathrm{kcal} / \mathrm{mol}$ Cartesian coordinates
$\begin{array}{lllll}N & \text { Atom } & X & Y & Z\end{array}$
$\begin{array}{lllll}1 & N & 0.0000 & -0.03060 & 0.0000\end{array}$
2 C $1.31150-0.03060-0.52470$
$\begin{array}{lllll}3 & C & -0.20140 & -0.03060 & 1.39820\end{array}$
4 C $\quad-1.11020-0.03060-0.87350$
5 C $\quad-2.21880 \quad-0.82590 \quad-0.60740$
$\begin{array}{lllll}6 & C & -1.12670 & 0.82590 & -2.01600\end{array}$
$\begin{array}{lllll}7 & C & 0.58340 & -0.82590 & 2.22520\end{array}$
$8 \quad$ C $\quad-1.18250 \quad 0.82590 \quad 1.98370$
9 C $1.63540-0.82590-1.61790$
$\begin{array}{lllll}10 & C & 2.30920 & 0.82590 & 0.03230\end{array}$
11 C $\quad-3.33160-0.03060-2.62860$
12 C $3.94230-0.03060-1.57090$
13 C $\quad-0.61070-0.03060 \quad 4.19960$
14 C $0.38190-0.82590 \quad 3.60330$
$\begin{array}{lllll}15 & C & -1.38880 & 0.82590 & 3.35800\end{array}$
16 C $2.92960-0.82590-2.13240$
$\begin{array}{lllll}17 & C & 3.60250 & 0.82590 & -0.47620\end{array}$
18 C $\quad-3.31150-0.82590 \quad-1.47090$
$\begin{array}{lllll}19 & C & -2.21370 & 0.82590 & -2.88180\end{array}$
20 C $-8.71950-0.15760-6.92620$
$21 \quad C \quad-1.63860 \quad-0.15760 \quad 11.0144$
22 C $10.3580-0.15760-4.08820$
23 C $\quad-6.48920-0.03060 \quad-5.14690$
24 C $7.70190-0.03060-3.04640$
$\begin{array}{lllll}25 & C & -1.21270 & -0.03060 & 8.19330\end{array}$ 


$$
\begin{aligned}
& \begin{array}{lllll}
26 & C & 10.0315 & 0.61370 & -2.95710
\end{array} \\
& 27 \text { C } 9.32020-0.82590-4.71350 \\
& \begin{array}{lllll}
28 & C & -2.45480 & 0.61370 & 10.1661
\end{array} \\
& 29 \text { C } \quad-0.57810 \quad-0.82590 \quad 10.4283 \\
& 30 \text { C } \quad-8.74210-0.82590 \quad-5.71480 \\
& 31 \text { C } \quad-7.57670 \quad 0.61370 \quad-7.20900 \\
& 32 \text { C } \quad-6.49790 \quad 0.61370 \quad-6.34610 \\
& 33 \text { C } \quad-7.64220-0.82590-4.85600 \\
& 34 \text { C } \quad-2.24690 \quad 0.61370 \quad 8.80040 \\
& \begin{array}{lllll}
35 & C & 8.74480 & 0.61370 & -2.45430
\end{array} \\
& 36 \text { C } 8.02650-0.82590 \quad-4.19030 \\
& \begin{array}{lllll}
37 & C & -0.38430 & -0.82590 & 9.04630
\end{array} \\
& 38 \text { C } \quad-1.80770-0.15760 \quad 12.4550 \\
& 39 \text { C } 11.6902-0.15760-4.66200 \\
& 40 \text { C } \quad-9.88250-0.15760-7.79300 \\
& 41 \text { C } 5.25440-0.03060-2.08660 \\
& 42 \text { C } \quad-4.43430-0.03060-3.50710 \\
& 43 \text { C } \quad-0.82010-0.03060 \quad 5.59380 \\
& 44 \text { C } \quad-10.0685 \quad 0.15760 \quad-8.98780 \\
& \begin{array}{lllll}
45 & C & 12.8180 & 0.15760 & -4.22570
\end{array} \\
& \begin{array}{lllll}
46 & C & -2.74940 & 0.15760 & 13.2135
\end{array} \\
& \begin{array}{llllll}
47 & C & -0.99890 & -0.03060 & 6.80560
\end{array} \\
& 48 \text { C } 6.39330 \quad-0.03060-2.53770 \\
& 49 \text { C } \quad-5.39430-0.03060-4.26790 \\
& \begin{array}{lllll}
50 & C & -2.91180 & 0.15760 & 14.6568
\end{array} \\
& \begin{array}{lllll}
51 & C & -11.2373 & 0.15760 & -9.85010
\end{array} \\
& 52 \text { C } \quad \begin{array}{llll}
14.1491 & 0.15760 & -4.80680
\end{array} \\
& \begin{array}{lllll}
53 & \mathrm{H} & 1.33080 & -1.56860 & 1.82450
\end{array} \\
& \begin{array}{lllll}
54 & \mathrm{H} & -0.28740 & 1.56860 & -2.23380
\end{array}
\end{aligned}
$$




$$
\begin{aligned}
& \begin{array}{lllll}
55 & \mathrm{H} & -2.24540 & -1.56860 & 0.24030
\end{array} \\
& \begin{array}{lllll}
56 & \mathrm{H} & 0.91460 & -1.56860 & -2.06470
\end{array} \\
& \begin{array}{lllll}
57 & \mathrm{H} & -1.79080 & 1.56860 & 1.36580
\end{array} \\
& \begin{array}{lllll}
58 & \mathrm{H} & 2.07820 & 1.56860 & 0.86800
\end{array} \\
& \begin{array}{lllll}
59 & \mathrm{H} & 4.36250 & 1.56860 & -0.03310
\end{array} \\
& 60 \quad H \quad 0.97560-1.56860 \quad 4.24860 \\
& \begin{array}{lllll}
61 & H & -2.15260 & 1.56860 & 3.79460
\end{array} \\
& 62 \text { H } \quad-4.16720-1.56860-1.27940 \\
& 63 \quad \mathrm{H} \quad-2.20990 \quad 1.56860 \quad-3.76150 \\
& 64 \quad H \quad 3.19160-1.56860-2.96920 \\
& 65 \text { C } \quad-12.4094-0.48160-9.54320 \\
& 66 \text { C } 14.4693-0.48160-5.97520 \\
& \begin{array}{lllll}
67 & C & -2.05990 & -0.48160 & 15.5184
\end{array} \\
& \begin{array}{lllll}
68 & N & 15.1208 & 0.82590 & -4.15480
\end{array} \\
& \begin{array}{lllll}
69 & N & -11.1586 & 0.82590 & -11.0175
\end{array} \\
& \begin{array}{lllll}
70 & N & -3.96220 & 0.82590 & 15.1724
\end{array} \\
& \begin{array}{lllll}
71 & C & -4.13990 & 0.82590 & 16.4878
\end{array} \\
& \begin{array}{lllll}
72 & \text { C } & -12.2089 & 0.82590 & -11.8292
\end{array} \\
& \begin{array}{lllll}
73 & C & 16.3488 & 0.82590 & -4.65860
\end{array} \\
& \begin{array}{lllll}
74 & \text { H } & 9.52820 & -1.56860 & -5.55880
\end{array} \\
& \begin{array}{lllll}
75 & \mathrm{H} & 10.7960 & 1.20510 & -2.46030
\end{array} \\
& \begin{array}{lllll}
76 & \mathrm{H} & 0.04990 & -1.56860 & 11.0310
\end{array} \\
& \begin{array}{lllll}
77 & H & -7.52870 & 1.20510 & -8.11950
\end{array} \\
& 78 \text { H } \quad-9.57810 \quad-1.56860 \quad-5.47230 \\
& \begin{array}{lllll}
79 & \mathrm{H} & -3.26730 & 1.20510 & 10.5797
\end{array} \\
& 80 \quad \text { H } \quad 0.41440-1.56860 \quad 8.61730 \\
& 81 \quad H \quad-2.87820 \quad 1.28010 \quad 8.17200 \\
& 82 \text { H } 7.25560-1.56860-4.66750 \\
& 83 \text { H } \quad-7.67000 \quad-1.56860 \quad-3.94980
\end{aligned}
$$



$\begin{array}{lllll}84 & H & -5.63800 & 1.28010 & -6.57860\end{array}$
$85 \quad$ H $\quad 8.51630 \quad 1.28010 \quad-1.59340$
86 C $\quad 16.6442 \quad 0.03060 \quad-5.80930$
$\begin{array}{llllll}87 & C & -3.29110 & 0.03060 & 17.3189\end{array}$
$\begin{array}{lllll}88 & C & -13.3531 & 0.03060 & -11.5097\end{array}$
89 H $11.7667-0.82590 \quad-5.53900$
$\begin{array}{lllll}90 & \mathrm{H} & -10.6803 & -0.82590 & -7.42080\end{array}$
$91 \quad H \quad-1.08640 \quad-0.82590 \quad 12.9598$
$92 \quad \mathrm{~N} \quad-13.4291-0.61370-10.5050$
$93 \quad$ N $15.8121-0.61370-6.37740$
$\begin{array}{lllll}94 & N & -2.38300 & -0.61370 & 16.8824\end{array}$
$\begin{array}{lllll}95 & H & -9.29190 & 0.93990 & -9.40580\end{array}$
$96 \quad$ H $\quad 12.7916 \quad 0.93990 \quad-3.34410$
$\begin{array}{lllll}97 & \mathrm{H} & -3.49970 & 0.93990 & 12.7499\end{array}$
$98 \quad H \quad-1.19920-0.93990 \quad 15.1336$
$99 \quad \mathrm{H} \quad-12.5064 \quad-0.93990 \quad-8.60530$
$100 \quad$ H $\quad 13.7057-0.93990-6.52820$
$\begin{array}{lllll}101 & H & -4.99110 & 1.56860 & 16.8960\end{array}$
102 H $\quad 17.1279 \quad 1.56860-4.12560$
$\begin{array}{lllll}103 & \text { H } & -12.1369 & 1.56860 & -12.7704\end{array}$
$104 \quad H \quad 17.6566 \quad 0.03060-6.20640$
$\begin{array}{lllll}105 & H & -3.45340 & 0.03060 & 18.3943\end{array}$
$\begin{array}{lllll}106 & H & -14.2032 & 0.03060 & -12.1879\end{array}$ 